\title{
WestVirginiaUniversity
}

THE RESEARCH REPOSITORY @ WVU

Graduate Theses, Dissertations, and Problem Reports

2009

\section{Functions of Rx in early vertebrate ocular development}

Brian G. Zamora

West Virginia University

Follow this and additional works at: https://researchrepository.wvu.edu/etd

\section{Recommended Citation}

Zamora, Brian G., "Functions of Rx in early vertebrate ocular development" (2009). Graduate Theses, Dissertations, and Problem Reports. 4554.

https://researchrepository.wvu.edu/etd/4554

This Dissertation is protected by copyright and/or related rights. It has been brought to you by the The Research Repository @ WVU with permission from the rights-holder(s). You are free to use this Dissertation in any way that is permitted by the copyright and related rights legislation that applies to your use. For other uses you must obtain permission from the rights-holder(s) directly, unless additional rights are indicated by a Creative Commons license in the record and/ or on the work itself. This Dissertation has been accepted for inclusion in WVU Graduate Theses, Dissertations, and Problem Reports collection by an authorized administrator of The Research Repository @ WVU.

For more information, please contact researchrepository@mail.wvu.edu. 


\title{
Functions of $\boldsymbol{R} x$ in Early Vertebrate Ocular Development
}

\author{
Brian G. Zamora \\ Dissertation submitted to the School of Medicine \\ at West Virginia University \\ in partial fulfillment of the requirements \\ for the degree of \\ Doctor of Philosophy \\ in \\ Biochemistry and Molecular Biology \\ Peter H. Mathers, Ph.D., Chair \\ Ashok Bidwai, Ph.D. \\ Fred Minnear, Ph.D. \\ Maxim Sokolov, Ph.D. \\ Visvanathan Ramamurthy, Ph.D. \\ Department of Biochemistry \\ Morgantown, West Virginia \\ 2009
}

Keywords: $R x$, eye, neural retina, Cre/lox $P$, conditional inactivation, fibroblast growth factor (FGF), development 


\title{
Abstract \\ Functions of $R x$ in Early Vertebrate Ocular Development
}

\author{
Brian G. Zamora
}

Patterning of the optic vesicle is a crucial step in early vertebrate eye development that organizes uncommitted optic vesicle cells into distinct distal, dorsal, and proximal regions that will give rise to the neural retina, retinal pigment epithelium (RPE), and optic stalk, respectively. Originating from the same sheet of anterior neuroectoderm, uncommitted optic vesicle cells are patterned into neural and non-neural (RPE) retinal domains through the coordinated activities of extrinsic signaling molecules and intrinsic transcription factors. Neural retinal specification is driven by FGF signals emanating from the surface ectoderm and developing lens, while RPE is specified by signals from the extraocular mesenchyme overlying the dorsal optic vesicle, likely a TGF- $\beta$ superfamily protein. Similarly, signaling from the optic vesicle has been implicated in directing lens formation from cells in the surface ectoderm. Various experiments have shown that patterning of neural retina and RPE can be altered through the ectopic introduction of signaling molecules, or the manipulation of various developmentally regulated transcription factors. Indeed, in frogs, chicks, and rodents, determination of neural retina and RPE can be interchanged for some time after their initial specification: presumptive RPE cells can transdifferentiate into neural retinal cells, and vice versa, demonstrating the bipotentiality of optic vesicle cells. Specifically, numerous studies demonstrate FGFs, and downstream effectors of FGF signaling, are important mediators of RPE-to-neural retinal transdifferentiation.

$R x$ is a paired-like homeobox gene that encodes a transcription factor that is expressed in retinal progenitor cells (RPCs) of the developing optic vesicle/cup. As the optic cup forms, $R x$ expression is restricted to the inner layer of developing optic cup (presumptive neural retina) and is terminated as retinal progenitors exit the cell cycle and differentiate into a neural retinal cell type. $R x$ expression is maintained through adulthood in Müller glial cells, which have been shown to function like neural retinal stem cells. During embryogenesis, mice homozygous for a targeted $R x$-null allele fail to form optic vesicles, demonstrating $R x$ is intrinsically required for the earliest stage of eye formation - evagination of the optic vesicles. Given the expression of $R x$ in proliferating retinal progenitors, we hypothesize that following optic vesicle evagination, $R x$ plays a role in promoting the proliferation of retinal progenitors, which could impact retinal and lens morphogenesis, and is required for the specification and/or maintenance of neural retinal identity.

In this dissertation, the functions of $R x$ during the early stages of vertebrate ocular development following optic vesicle evagination are ascertained through $R x$ loss-of-function studies using Cre/loxP conditional inactivation strategies. We demonstrate that inactivation of $R x$ during the optic vesicle stage, via the Foxg1-Cre animal model, arrests retinal progenitor cell proliferation and prevents neural retinal specification, generating an optic vesicle remnant composed entirely of RPE-fated cells. Further, genetic introduction of an FGF9 transgene, which can promote transdifferentiation of RPE to neural retina, into this optic vesicle-inactivated $R x$ model is unable to rescue neural retinal formation, suggesting $R x$ is required during the optic vesicle stage to initiate neural retinal specification. Through the use of the Six3-Cre transgenic animal model, we show that inactivation of $R x$ during optic cup development also impedes 
retinal progenitor cell proliferation. Due to delayed onset of Cre expression in the Six3-Cre line compared to Foxg1-Cre, this $R x$ conditional inactivation model allows for the initiation of optic cup morphogenesis and lens induction, but remains unable to specify neural retinal identity. This model indicates $R x$ may have roles during optic cup development in RPC proliferation, optic cup morphogenesis and lens induction that are separate from its role in neural retinal specification. Remarkably, introduction of the FGF9 transgene into this optic cup-inactivated $R x$ model can initiate neural retinal specification and formation, but is unable to maintain neural retinal identity. This compound mutant model suggests delayed $R x$ inactivation (i.e.- prolonged expression) in the developing optic cup confers competence to undergo FGF-mediated neural retinal specification in $R x$-depleted cells. We propose a model in which $R x$ activity is absolutely required to specify neural retinal cell identity until a temporal or developmental threshold is met during the optic cup stage; after which neural retinal cells can be specified via FGF-signaling, independent of $R x$ activity.

Overall, the studies contained within this dissertation indicate the $R x$ gene has functional roles in RPC proliferation, optic cup and lens morphogenesis, and neural retinal specification, and also uncovers a novel role for $R x$ in determining retinal progenitor cell competence to undergo FGF-mediated RPE-to-neural retinal transdifferentiation. 


\section{Acknowledgements}

I would like to thank my advisor, Dr. Peter Mathers, for sharing his knowledge and expertise over the years. I am grateful to have been able to work on multiple fulfilling projects and manage and develop them with great independence. With his help and guidance, I was able to hone my skills as an independent scientist, a critical thinker, and an effective writer.

I would also like to thank the members of my committee, Dr. Ashok Bidwai, Dr. Fred Minnear, Dr. Visvanathan Ramamurthy, and Dr. Maxim Sokolov, for all their advice, help and support throughout my graduate career. Besides lending their technical and scientific expertise and advice, each provided unique examples of how to have a successful career in science and academia, which are lessons I will take with me as I develop my career in the future.

Finally, I would like to thank all of my colleagues in the Mathers lab, past and present, for their technical expertise and help with my projects. I would like to thank all other graduate students for sharing their knowledge and experience, and for providing support throughout my research career. I am extremely grateful for the love, support and encouragement from friends and family, who helped keep me grounded throughout my time in the lab. 


\section{Table of Contents}

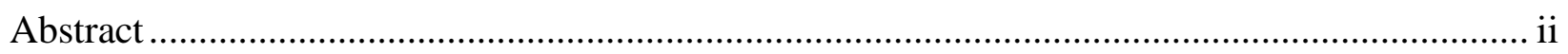

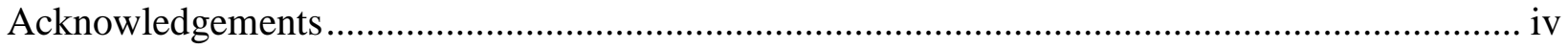

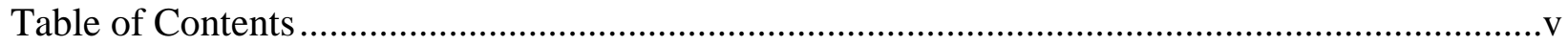

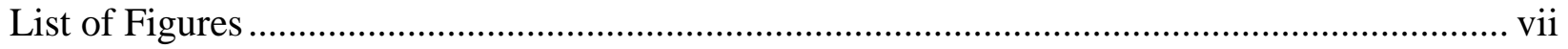

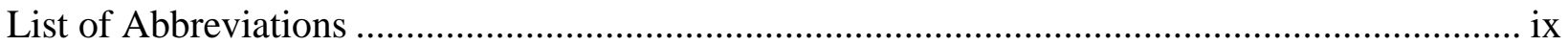

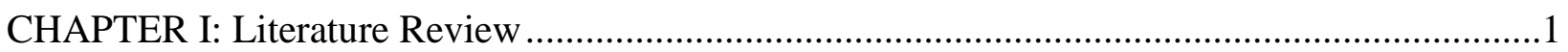

A. Early vertebrate eye development...............................................................................

B. Lens placode and optic vesicle communication...............................................................6

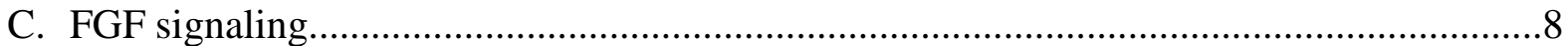

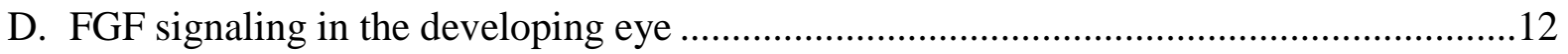

E. Transdifferentiation of retinal pigment epithelium (RPE) to neural retina.........................15

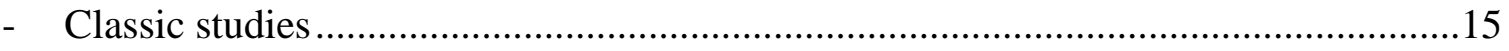

- FGFs and FGF signaling ..................................................................................16

- Transcription factors and other mechanisms of RPE-to-neural retinal

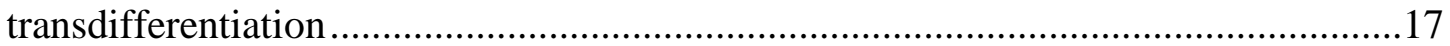

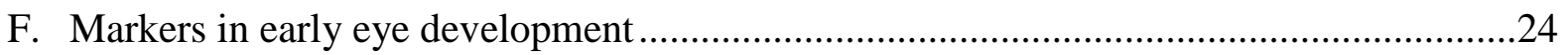

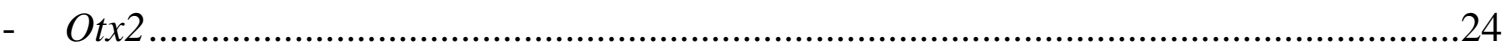

- Six

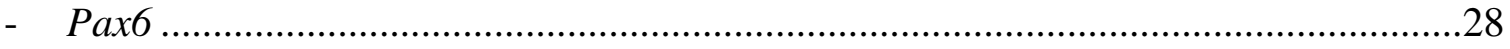

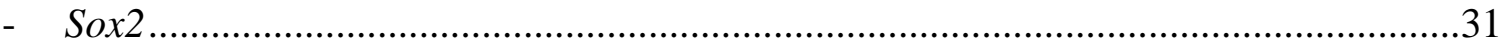

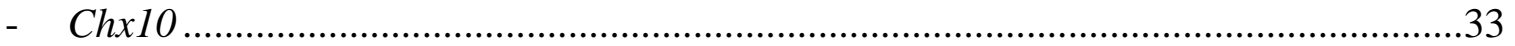

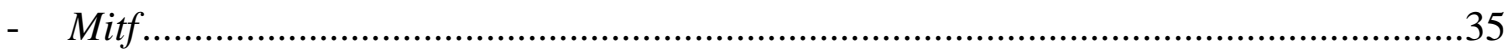

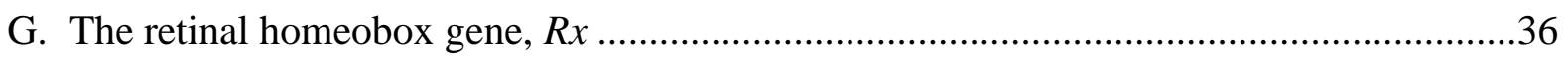

- History of $R x$ isolation ..........................................................................................

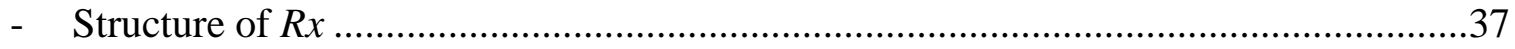

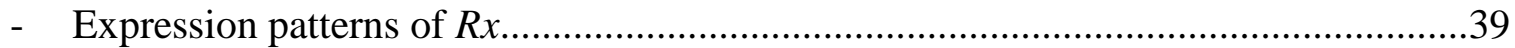

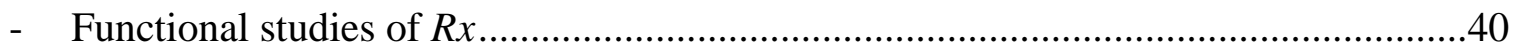

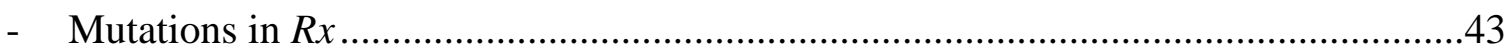

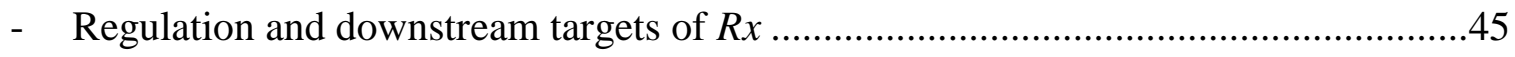


H. Site specific DNA recombination systems: Cre/loxP and FLP/Frt .................................46

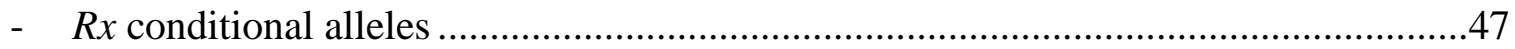

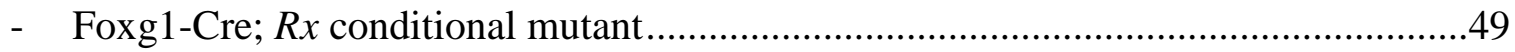

CHAPTER II: $R x$ is required for specification of the neural retina, independent of optic cup morphogenesis and lens induction ....................................................................................

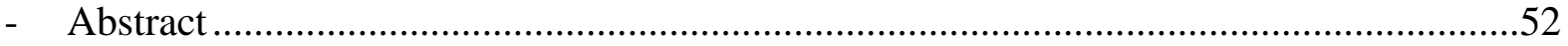

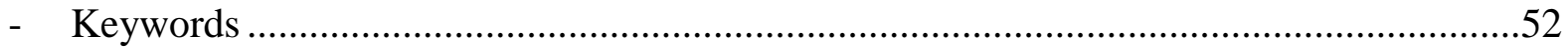

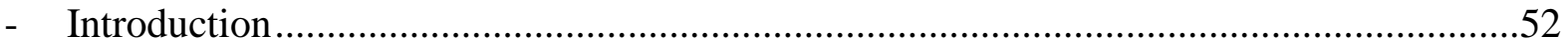

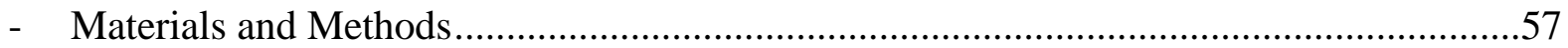

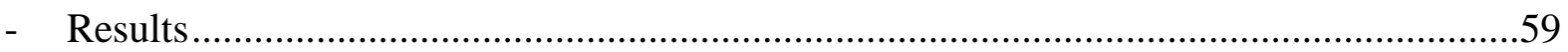

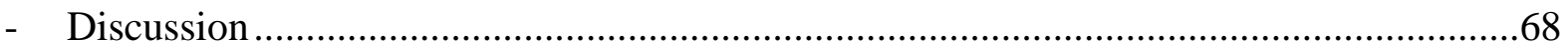

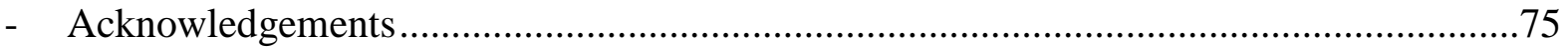

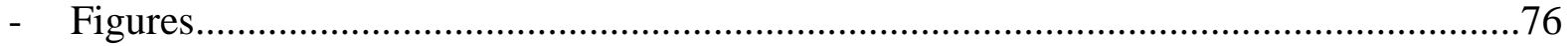

CHAPTER III: Temporal requirement of $R x$ for FGF-mediated neural retinal specification ........89

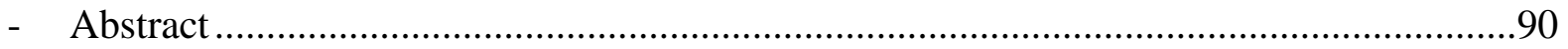

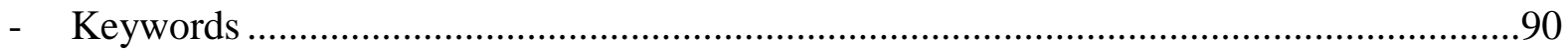

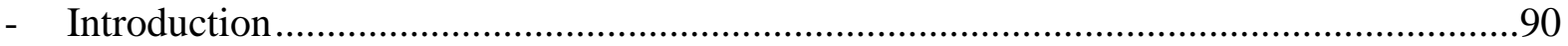

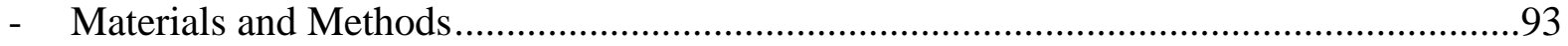

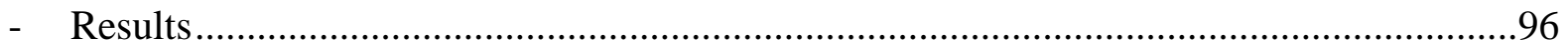

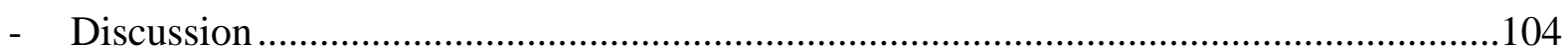

- Acknowledgements ...........................................................................................110

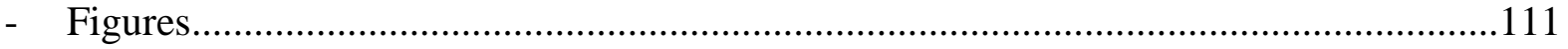

CHAPTER IV: Summary, Implications, and Future Directions...................................................123

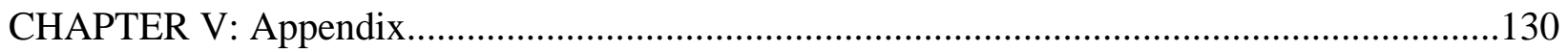

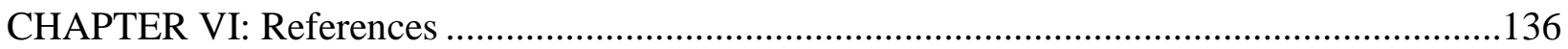




\section{List of Figures}

CHAPTER I: Literature Review

Figure 1. Early eye development and retinal composition ...............................................5

Figure 2. FGFR Structure and the FGF signaling pathway ..........................................11

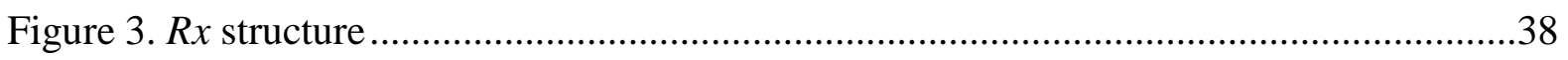

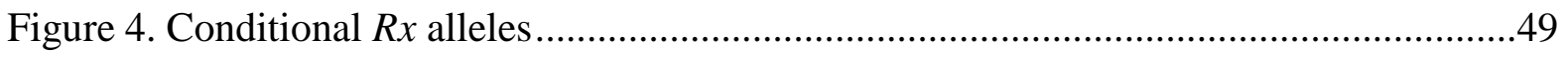

CHAPTER II: $R x$ is required for specification of the neural retina, independent of optic cup morphogenesis and lens induction

Figure 1. Six3-Cre-mediated conditional inactivation of $R x$ during the optic cup stage of development causes postnatal anophthalmia with a pigmented cystic remnant in the orbit and concomitant loss of optic nerves and optic chiasm

Figure 2. Six3-Cre-mediated inactivation of $R x$ in the optic cup creates a range of early eye developmental phenotypes spanning severely to mildly affected eyes

Figure 3. Fate change for Six3-Cre; $R x$ conditional mutant cells ....

Figure 4. Decreased proliferation in the developing eye tissue of Six3-Cre; $R x$ conditional mutants versus control littermates

Figure 5. Decreased mitosis and total cell number in Six3-Cre; $R x$ conditional mutant optic vesicle remnants

Figure 6. Six3-Cre-mediated deletion of $R x$ prevents the specification of neural retinal tissue

Figure 7. The moderate and mild phenotypes of the Six3-Cre; $R x$ conditional mutant allow for the initial specification of lens placode and lens vesicle, respectively

Figure 8. Six3-Cre-mediated inactivation of $R x$ results in a persistent optic vesicle/cup that adopts an RPE cell fate

Figure 9. Model for the generation of variable developing eye phenotypes in Six3-

Cre; $R x$ conditional mutant embryonic eyes

Supplemental Figure S1. Inconsistent Cre-mediated recombination/EGFP reporter activation in E9.5 optic vesicles by Six3-Cre transgenic model.

Supplemental Figure S2. No cell death in the developing eyes of E10.5 and E11.5 Six3-Cre; $R x$ conditional mutants 
CHAPTER III: Temporal requirement of $R x$ for FGF-mediated neural retinal specification

Figure 1. Ectopic FGF signaling fails to rescue ocular morphology of the Foxg1-

Cre; $R x$ conditional mutant model

Figure 2. Cells of the $R x$-deleted lineage remain present in the optic remnants of Foxg1-Cre; $R x^{\text {flox }}$; FGF9 compound mutants.

Figure 3. Ectopic FGF signaling (MEK/ERK pathway) via the OVE1070 transgene is maintained in the Foxg1-Cre; $R x^{\text {flox }}$; FGF9 compound mutant optic vesicle remnant

Figure 4. Ectopic FGF signaling fails to rescue neural retinal specification in Foxg1-Cre; $R x$ conditional mutants, and the compound mutant optic vesicle remnant maintains an RPE fate.

Figure 5. Addition of ectopic FGF signaling to Six3-Cre; $R x$ conditional mutant partially rescues developing eye morphology....

Figure 6. RPE identity of the Six3-Cre; $R x$ conditional single mutant eye is lost with the introduction of ectopic FGF signaling

Figure 7. Neural retinal cell fate is respecified with the addition of ectopic FGF9 signaling to the Six3-Cre; $R x$ conditional mutant model

Figure 8. FGF-mediated neural retinal respecification can occur in the absence of $R x$ activity

Figure 9. Neural retinal respecification in the distal-ventral, $R x$-depleted region surrounding the developing lens correlates with lens vesicle-dependent FGF9 signaling and ERK activation.

Figure 10. Model for phenotypic outcomes of Foxg1-Cre; $R x^{\text {flox }}$ FGF9 and Six3Cre; $R x^{\text {flox }}$; FGF9 compound mutants

Chapter IV: Appendix

Figure 1. The Trp2-FLAG-hRX transgenic animal model ..... 


\section{List of Abbreviations}

aa

bp

bHLH

bHLHZip

BMP

CKO

CNS1

DIG

E

EGFP

ERK

ES

eyl

FGF

FGFR

FLP

Frt

GCL

GFP

$\mathrm{Hh}$

HMG

HPE

HSPG

$\mathrm{Ig}$

INL

iPS

LP

LV

MAPK

MEK

$m i$

Mitf

MS

neo

NR

ONL

$o r^{J}$

OS

OV

$\mathrm{P}$

PBS

PCNA

PCE-1 amino acid

base pair(s)

basic helix-loop-helix

basic helix-loop-helix zipper

bone morphogenetic protein

conditional knockout

conserved non-coding sequence 1

digoxigenin

embryonic age

enhanced green fluorescent protein

extracellular-regulated kinase

embryonic stem

mouse eyeless allele

fibroblast growth factor

fibroblast growth factor receptor

flippase recombinase

FLP recognition target

ganglion cell layer

green fluorescent protein

hedgehog

high mobility group

holoprosencephaly

heparan sulfate proteoglycan

immunoglobulin

inner nuclear layer

induced pluripotent stem

lens placode

lens vesicle

mitogen-activated protein kinase

MAP/ERK kinsae

microphthalmia allele

Microphthalmia-associated transcription factor

mesenchyme

neomycin resistance cassette

neural retina

outer nuclear layer

ocular retardation allele

optic stalk

optic vesicle

postnatal age

phosphate buffered saline

proliferating cell nuclear antigen

photoreceptor conserved element-1 


$\begin{array}{ll}\text { PCR } & \text { polymerase chain reaction } \\ \text { pERK } & \text { phosphorylated extracellular-regulated kinase } \\ \text { PG } & \text { pigment granules } \\ \text { pHH3 } & \text { phosphorylated histone H3 } \\ \text { PST } & \text { proline, serine, threonine } \\ \text { PI } & \text { propidium iodide } \\ \text { RPC } & \text { retinal progenitor cell } \\ \text { RPE } & \text { retinal pigment epithelium } \\ \text { SE } & \text { surface ectoderm } \\ \text { Sey } & \text { small eye allele } \\ \text { Shh } & \text { sonic hedgehog } \\ \text { TCF/LEF } & \text { T-cell factor/lymphoid enhancer factor } \\ \text { TGF- } \beta & \text { transforming growth factor-beta }\end{array}$




\section{Chapter 1: Literature Review}




\section{A. Early vertebrate eye development}

The studies of developmental biology and embryology have at their core the desire to understand the mechanisms responsible for the coordinated development of tissues and organs. Ever since the early experiments of Spemann, first conducted over a hundred years ago in the early 1900s, eye development has long been studied as a model for understanding the inductive interactions that must be coordinated for the proper formation of our visual organ.

A functional eye develops from the interaction and fusion of two distinct early embryonic components: the neural ectoderm and the surface ectoderm, which simplistically, give rise to the retina and the lens, respectively. Development of the eye first starts with the specification of cells within the anterior neural plate, which make up the eye field. The eye field is then split along the midline through sonic hedgehog signaling emanating from the prechordal plate, to form two retinal domains, from which the retina will rise (Li et al., 1997).

The first morphologic sign of eye formation in vertebrates is the bilateral evagination of the two retinal domains of the anterior neural plate/ventral forebrain to form the optic pits, which occurs around mouse embryonic day (E)8.5. The optic pits grow laterally to become optic vesicles, which eventually make contact with the non-neural surface ectoderm on E9.5 (Fig. 1A; reviewed in Chow and Lang, 2001). As the optic vesicle makes contact with the surface ectoderm, it becomes partitioned into three territories: a distal territory that will become neural retina; a proximal territory that will become optic stalk, and a dorsal territory that will become the retinal pigment epithelium (RPE). The interaction of the optic vesicle and the overlying surface ectoderm initiates a cascade of events critical for the specification of the eye's most important components - the neural retina and lens — and is a developmental timepoint of interest for much of this work. 
Inductive signals from the neural ectoderm-derived optic vesicle stimulate thickening of surface ectoderm to form lens placode, which consequently invaginates and pinches off from the surrounding surface ectoderm to form the lens vesicle. At the same time, interactive signals from the surface ectoderm-derived lens placode induce thickening and invagination of the distal optic vesicle abutting the lens placode, to form a bilayered optic cup, whose inner layer (next to the lens) forms neural retina and outer layer forms the RPE (reviewed by Chow and Lang, 2001). The optic cup is first evident in the mouse embryo at around E10.

The dorsal portion of the optic vesicle receives signals from the surrounding extraocular mesenchyme that direct its differentiation into RPE (Fuhrmann et al., 2000). The most proximal portion of the optic vesicle, connecting the forebrain to the developing retina, narrows and elongates to become the optic stalk, the conduit through which the optic nerve (retinal ganglion cell axons) will travel to make connections with the brain. Invagination of the optic vesicle creates a groove that runs along the ventral axis of the neural retina and optic stalk. The growing edges of the optic cup eventually fuse, and this groove creates the optic or choroidal fissure, through which blood vessels and retinal ganglion axons can exit the optic cup.

Once the lens vesicle and the double-layered optic cup are established, this general morphology is maintained throughout development to the adult eye. However, important changes still take place. Briefly, cells of the lens vesicle proliferate and differentiate into fiber cells that lose their organelles, creating a translucent, mature lens. Soon after optic cup morphogenesis, the RPE begins melanin pigment biosynthesis and deposition, giving the developing mouse eye its dark color. The RPE maintains a low level of proliferation and expansion to keep up with the growth of the neural retina, but preserves its organization as a 
cuboidal monolayer of epithelial cells, sandwiched between the photoreceptors of the neural retina and the vascular meshwork of the choroid layer.

During vertebrate optic cup morphogenesis, the developing neural retina is composed of neural retinal progenitors that proliferate and expand the overall size of the developing neural retina. These progenitors eventually exit the cell cycle, terminally differentiate into one of six neural retinal cell types or a glial cell type, and migrate to a pre-determined position dependent on their cell type. These post-mitotic retinal neurons are organized into three distinct cellular layers, giving the retina its laminar structure. In addition, neural retinal cell types exit the cell cycle and differentiate in a relatively fixed chronological sequence (Fig. 1B; reviewed in Marquardt and Gruss, 2002). This cellular differentiation first begins in the inner layer of the central optic cup and progresses radially towards the peripheral edges of the retina in a wave-like fashion. Ganglion cells are the first retinal cell type to differentiate and are considered "earlyborn" retinal cell types along with cones, and horizontal and amacrine cells. The remaining retinal cell types_rods, bipolar cells, and Müller glia—are considered "late-born" and begin terminal differentiation several days after the early-born retinal cell types (reviewed by Pearson and Doe, 2004). These seven retinal types can be categorized into glial cells (Müller glial cells) and neuronal cells, which can be further subdivided into three different classes: light-sensitive photoreceptors (rods and cones), whose nuclei make up the outer nuclear layer, and which reside just inside the RPE layer; interneurons (bipolar, horizontal, and amacrine cells), which make up the inner nuclear layer; and retinal ganglion cells, which, along with displaced amacrine cells, make up the inner-most ganglion cell layer next to the vitreous (Fig. 1C). It is the coordinated activities of these seven retinal cell types, which are an extension of the central nervous system, that give vertebrates the sense of visual perception. 
A
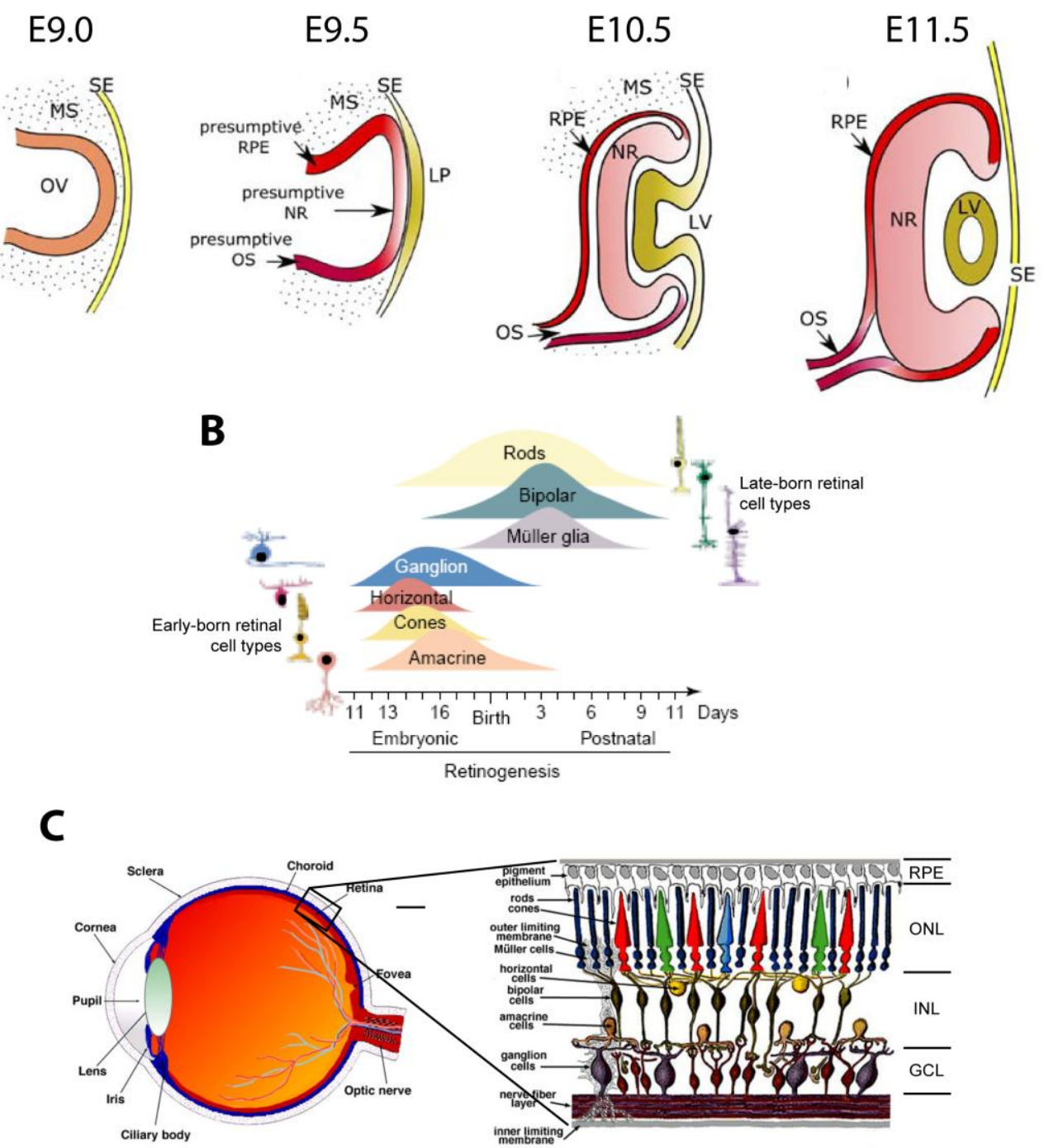

Figure 1. Early eye development and retinal composition.

(A) Early eye formation in the mouse eye. At mouse E9.0, the unpatterned optic vesicle (OV) is growing from the anterior neuroectoderm towards the surface ectoderm (SE). At E9.5, the OV makes contact with the SE, which thickens to form the lens placode (LP). Concurrently, the OV is patterned into its presumptive parts through signals from adjacent tissues. At E10.5, the optic cup is taking shape through the invagination of the distal OV and LP. At E11.5, the invaginating LP has pinched off to form the lens vesicle (LV) and the inner and outer layer of the optic cup form the neural retina (NR) and retinal pigment epithelium (RPE), respectively. MS, mesenchyme; OS, optic stalk (adapted from Adler \& Canto-Soler, 2007).

(B) Timeline and distribution of retinal cell type birth during retinogenesis (adapted from Marquardt \& Gruss, 2002).

(C) General structure of the fully developed eye and retina. RPE, retinal pigment epithelium; ONL, outer nuclear layer; INL, inner nuclear layer; GCL, ganglion cell layer (adapted from http://webvision.med.utah.edu/anatomy.html). 


\section{B. Lens placode and optic vesicle communication}

Early induction studies established the importance of contact between the distal optic vesicle and the presumptive lens ectoderm for the proper development of the neural retina and lens. First, tissue explantation experiments revealed that if the surface ectoderm overlying the optic vesicle was removed, the neural retina would not develop (Holtfreter, 1939). These initial observations have been repeated more recently and demonstrate the inductive importance of the surface ectoderm for normal neural retinal development (Hyer et al., 1998; Nguyen and Arnheiter, 2000). Moreover, in 1937, Ikeda reported that the transplantation of presumptive lens ectoderm could induce retinal differentiation from the RPE layer in eyes where the neural retina was removed (Ikeda, 1937). Similarly, in 1939, Mikami reported that the $180^{\circ}$ reversal of the optic vesicle, such that the presumptive RPE was facing the surface ectoderm, would transform the presumptive RPE into a secondary neural retina (Mikami, 1939).

More recent studies have demonstrated fibroblast growth factor (FGF) signaling is a key player mediating these inductive interactions between the lens placode and optic vesicle. It has been established that the pre-lens ectoderm is a source of FGFs-FGF1 and FGF2 (de Iongh and McAvoy, 1993), and that exogenous FGF is sufficient to specify neural retina in the absence of the pre-lens ectoderm (Hyer et al., 2003; Hyer et al., 1998; Nguyen and Arnheiter, 2000). Furthermore, addition of FGF2 neutralizing antibodies to optic vesicle explant cultures blocks the formation of neural retina (Pittack et al., 1997). Taken together, these lines of evidence implicate FGFs emanating from the lens placode as a signaling cue for neural retinal specification in the abutting distal optic vesicle.

The duration of the interaction between the optic vesicle and surface ectoderm, however, demonstrates inductive signals from the surface ectoderm can mediate both optic cup 
morphogenesis as well as neural retinal specification. Removal of the pre-lens ectoderm (when optic vesicle and surface ectoderm are apposed, but before lens placode forms) blocks optic cup morphogenesis, but still initiates specification of neural retina, while removal of the lens placode allows both optic cup morphogenesis and neural retinal specification (Hyer et al., 2003). Interestingly, while the addition of exogenous FGF to surface ectoderm-ablated optic vesicles can rescue neural retinal specification, those optic vesicles never develop an optic cup (Hyer et al., 2003; Hyer et al., 1998). Thus, while FGF from the surface ectoderm can induce neural retinal specification, the inductive signal from the surface ectoderm that is responsible for optic cup morphogenesis is probably not FGF.

The classic embryonic ablation studies of Spemann, in which he removed the presumptive retinal region of neural plate-stage Rana temporaria embryos and observed a loss of lens formation (Spemann, 1901), were the first experiments to demonstrate the idea of embryonic induction and also first established the potential significance of optic vesicle-derived inductive signals required for lens formation from the head surface ectoderm. While many of the specific signaling factors mediating lens induction have yet to be identified, current evidence points to FGF (Faber et al., 2001) and bone morphogenetic protein (BMP) signaling pathways (Dudley et al., 1995; Furuta and Hogan, 1998; Wawersik et al., 1999) playing a role in lens induction and participating in the inductive communication from the optic vesicle to the overlying surface ectoderm (reviewed in Lang, 2004; and Lovicu and McAvoy, 2005). It is clear that proper development of the neural retina and lens requires the reciprocal inductive signals between the neuroepithelium-derived optic vesicle/cup and the head surface ectoderm-derived lens placode/vesicle, but the factors mediating these effects are not completely understood and remain an area of active research, to which the work contained herein contributes. 


\section{FGF signaling}

Fibroblast growth factors (FGFs) are one of several classes of cell-extrinsic, polypeptide growth factors that play an important role in embryonic organ development. The first FGF was discovered as a mitogen for cultured fibroblasts, and since then, at least 22 distinct FGFs have been identified in a variety of organisms (Ornitz and Itoh, 2001). In the mouse, the FGF protein family comprises 22 members, FGF1-FGF23, excluding FGF19 (a human ortholog of mouse FGF15). All FGF family members share a high affinity for heparin as well as a conserved central core sequence of 120 amino acids (Ornitz and Itoh, 2001), which interacts with the FGFR (Plotnikov et al., 2000). FGFs range in size from 17 to $34 \mathrm{kDa}$, and most FGFs (FGFs 3-10, 1621, and 23) are secreted from cells (Itoh and Ornitz, 2004). In general, FGFs canonically mediate their cellular responses by binding to and activating a family of four transmembrane receptor tyrosine kinase proteins, designated the high-affinity FGF receptors FGFR1-FGFR4 (Reuss and von Bohlen und Halbach, 2003). FGFs also bind heparin or heparan sulfate proteoglycans (HSPGs), which are required for high affinity binding of FGF to FGFR, and FGF signal activation. (Rapraeger et al., 1991; Yayon et al., 1991).

The four FGFRs are single spanning transmembrane proteins, each composed of about 800 amino acids (Itoh and Ornitz, 2004). Like all receptor tyrosine kinases, FGFRs have 3 main domains: an extracellular ligand-binding domain; a hydrophobic transmembrane domain; and a cytoplasmic domain with a split catalytic tyrosine kinase domain, as well as additional regulatory sequences (Fig. 2; Johnson et al., 1990; Lee et al., 1989). The extracellular ligand-binding domain consists of two or three immunoglobulin (Ig)-like domains (IgI, IgII, and IgIII), which are required for FGF binding and confer binding affinity and ligand specificity. A stretch of 
acidic amino acids (acidic box domain) is located between Ig domains I and II, and a heparinbinding domain resides in IgII (Bottcher and Niehrs, 2005).

Various FGFR isoforms have been shown to arise from alternative splicing events occurring in both the intracellular and extracellular regions of FGFRs (Mohammadi et al., 2005). Importantly, alternative splicing in the third Ig-like domain (IgIII) of FGFRs 1-3, but not 4, alters ligand-binding specificity (Miki et al., 1992; Yayon et al., 1992). As an example, exon 7 of FGFR2 encodes for the N-terminal half of IgIII, while exons 8 and 9 alternatively encode for the C-terminal half of IgIII. The exon 7-8 splice variant of FGFR2 (FGFR2b) confers binding to FGF7 and FGF10, but not FGF2, and the exon 7-9 splice variant (FGFR2c) binds FGF2 and FGF18, but not FGF7 or FGF10, demonstrating how it is possible that just four FGFR genes can mediate diverse signals from the 22 different FGF family members (Miki et al., 1992; Yayon et al., 1992). Furthermore, it has been shown that the FGFR2b isoform is exclusively expressed in epithelial cells, and that the FGFR2c isoform is only expressed in mesenchymal cells, suggesting a tissue specific mechanism by which FGFR signaling can regulate different cellular effects (Orr-Urtreger et al., 1993).

The consensus mechanism by which FGFRs mediate a biochemical response begins with the dimerization of FGFRs in the plasma membrane, which is induced by FGF ligand binding and requires heparin or heparan sulfate proteoglycans (Fig. 2). Heparin, through its binding of FGF, is thought to act as a linker that is required for the dimerization of two independent FGF/FGFR complexes (Stauber et al., 2000). Once this complex is formed, interaction of the two cytoplasmic domains of the receptor dimer is thought to stimulate trans-autophosphorylation of tyrosine residues. Autophosphorylation sites in the catalytic domain are crucial for activation of the tyrosine kinase domains, while autophosphorylation sites in other locations are involved in 
the recruitment and assembly of signaling complexes (Klint and Claesson-Welsh, 1999;

Schlessinger, 2000). For example, phosphorylated tyrosines in the cytoplasmic domain of FGFRs can function as binding sites for Src homology 2 (SH2) and phosphotyrosine binding (PTB) domains of signaling proteins, which can then be activated by receptor tyrosine kinase-mediated phosphorylation (Forman-Kay and Pawson, 1999; Pawson et al., 1993). Alternatively, receptorbound proteins can serve as adaptors for other FGF signal transduction proteins independent of FGFR-mediated tyrosine phosphorylation (Forman-Kay and Pawson, 1999; Pawson et al., 1993). Both of these mechanisms are used by FGFRs to initiate FGF signal transduction cascades and carry out various cellular processes.

Three main signaling pathways mediate FGF signal transduction: (a) the RAS/MAPK pathway, (b) the PLC $\gamma / \mathrm{Ca}^{2+}$ pathway, and (c) the PI3 kinase/Akt pathway (reviewed in Bottcher and Niehrs, 2005). The most commonly activated and well studied signal transduction pathway mediated by FGFRs is the RAS/MAPK pathway, which involves the phosphotyrosine-mediated binding of the docking protein FRS2 (Kouhara et al., 1997), which recruits the GRB2/SOS complex to the plasma membrane (Fig. 2). The guanine nucleotide exchange factor, SOS, then activates the small G protein, RAS, which triggers the sequential phosphorylation of the protein kinases RAF, MEK, and ERK (MAPK). Activated ERK (extracellular-regulated kinase) is rapidly translocated to the nucleus to phosphorylate and activate transcription factors, which can exert various cellular effects (Yang, 2004). 


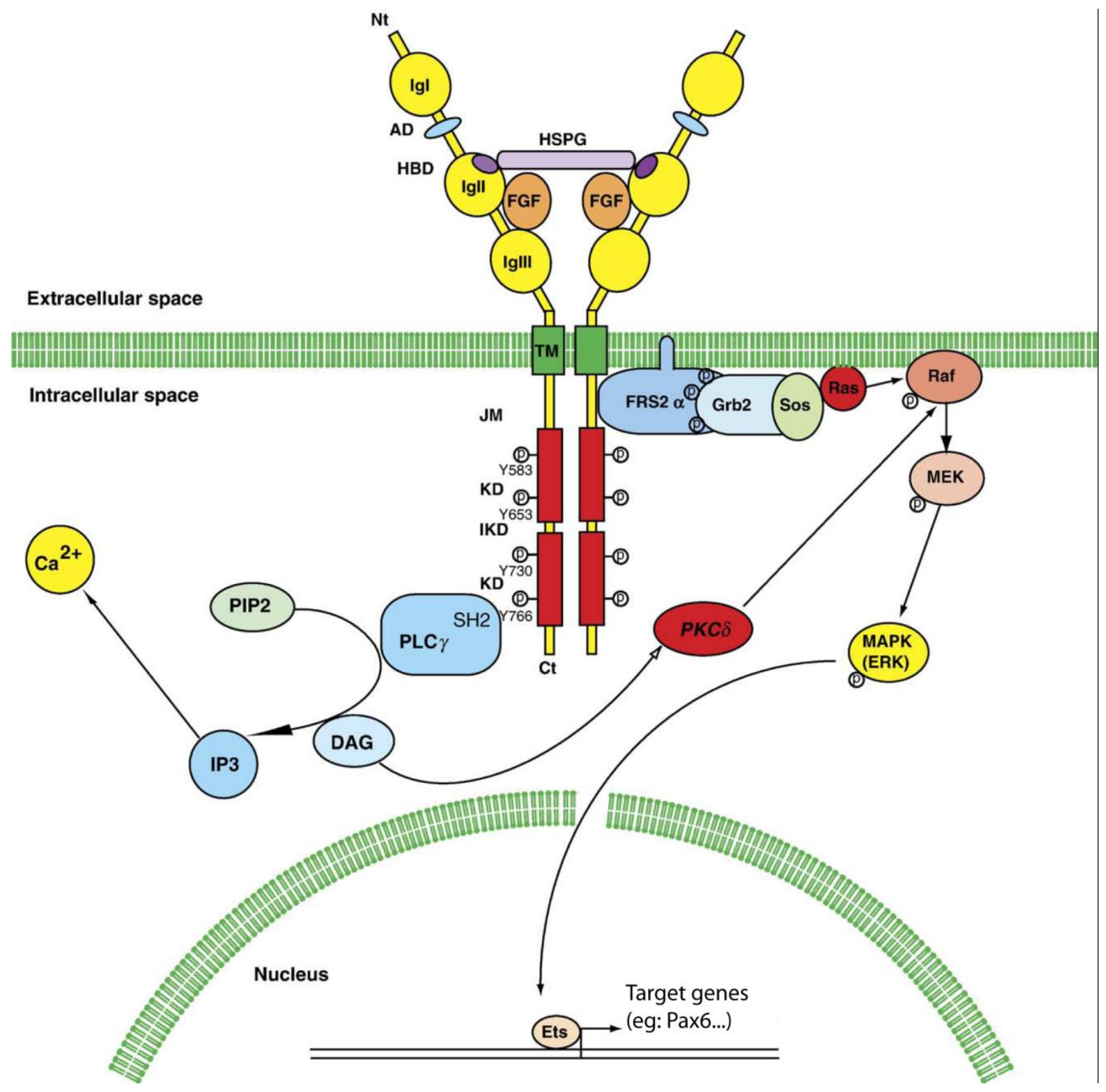

Figure 2. FGFR structure and the FGF signaling pathway. Fibroblast growth factors (FGFs), with the help of heparan sulfate proteoglycans (HSPG), bind the extracellular lg-like domains of FGF receptors, triggering receptor dimerization. Upon dimerization, the intracellular tyrosine kinase domains autophosphorylate each other and initiate a signal transduction cascade that ultimately leads to changes in gene expression within the nucleus (adapted from Thisse \& Thisse, 2005). 


\section{FGF signaling in the developing eye}

Embryonically, FGF signaling plays critical roles in morphogenesis by regulating cell proliferation, differentiation, and migration (Bottcher and Niehrs, 2005; Thisse and Thisse, 2005). In the embryonic vertebrate eye, FGF signaling functions in multiple roles. In addition to optic vesicle patterning, neural retinal specification and lens induction, as described above, FGF signaling is involved in retinal progenitor maintenance, retinal cell fate specification and differentiation, lens fiber differentiation, lens vesicle polarity, neuronal axon growth, retinal ganglion cell guidance and targeting, and retinal vascularization (reviewed in Lovicu and McAvoy, 2005; Robinson, 2006; Rousseau et al., 2003; Russell, 2003; and Yang, 2004). Of the 22 FGF family members, 13 (FGF1-3, 5, and 7-15), have been shown to be expressed in the vertebrate eye, either during development and/or at maturity (Robinson, 2006). For this review, I will focus the discussion to FGF family members involved in the embryonic development of vertebrate lens and retina.

FGF1 and FGF2 are expressed in the surface ectoderm and the developing lens, suggesting a role for FGFs in lens development. Exogenous application of FGF1 and FGF2 induces lens cells to migrate, proliferate and differentiate in a dose-dependent manner (McAvoy and Chamberlain, 1989). Similarly, transgenic mice ectopically expressing FGF4, 7, 8 or 9 in the lens undergo early fiber cell differentiation (Lovicu and Overbeek, 1998), and exogenous application of FGF8 to the developing eye induces lens fiber cell differentiation, and can even induce lens formation if applied early enough (Vogel-Hopker et al., 2000). In addition, expression of dominant-negative or dimerized soluble forms of FGFRs in developing fiber cells inhibits lens fiber differentiation (Chow et al., 1995; Govindarajan and Overbeek, 2001; Robinson et al., 1995). Correspondingly, disruption of FGF signaling in the presumptive lens 
ectoderm by small molecule FGFR kinase inhibitors or a dominant-negative FGFR results in abnormal lens induction and reduced expression of lens induction markers (Faber et al., 2001).

In vivo and in vitro exposure of RPE to either FGF1 or FGF2 trandifferentiates the RPE to neural retina (Guillemot and Cepko, 1992; Hyer et al., 1998; Nguyen and Arnheiter, 2000; Park and Hollenberg, 1989; Park and Hollenberg, 1991; Pittack et al., 1991; Sakaguchi et al., 1997; Spence et al., 2007; Zhao et al., 1997; Zhao et al., 1995) and provided the first lines of evidence that FGFs could function in neural retinal differentiation. More recently, FGF3, 8, 9 and 15 have been identified as FGF family members expressed early in the inner layer of the optic cup (Colvin et al., 1999; Martinez-Morales et al., 2005; McWhirter et al., 1997; VogelHopker et al., 2000; Zhao et al., 2001), suggesting these family members could play a more direct role in neural retinal development. Similar to FGF1 and FGF2, exogenous application of FGF8 to developing chick optic cups or transgenic expression of FGF9 in the presumptive RPE of embryonic mice, converts the presumptive RPE into neural retina (Martinez-Morales et al., 2005; Vogel-Hopker et al., 2000; Zhao et al., 2001; Zhao and Overbeek, 1999). FGF3 and FGF8 expression in the zebrafish optic cup correlates with the initiation and propagation of the neural differentiation wave in the developing retina (Martinez-Morales et al., 2005). Interestingly, zebrafish embryos that carry mutant FGF3 or FGF8 show no delays or defects in retinal development (Herzog et al., 2004; Shanmugalingam et al., 2000), while double mutant zebrafish embryos fail to initiate neural retinal differentiation (Martinez-Morales et al., 2005). In mouse embryos lacking FGF9, the RPE extends into presumptive neural retina (the inner layer of the optic cup), suggesting FGF9 functions to define the boundary between the neural retina and RPE, and that FGF9 is sufficient, but not necessary, to specify the neural retinal cell fate (Zhao et al., 2001). While the complete story of how FGFs in the retina are coordinated and regulated to 
promote proper neural retinal formation, it is clear that FGFs play an important role in neural retinal differentiation.

Mutational studies of the docking protein FRS2 $\alpha$ highlight the importance of FGF signal transduction in both lens induction and neural retinal differentiation (Gotoh et al., 2004). FRS2 $\alpha$ is a FGFR tyrosine kinase target protein that serves as the link between activated FGFR and the Ras/ERK and the PI3 kinase/Akt signal transduction pathways by its association with the protein tyrosine phosphatase, Shp2, and the adaptor protein, Grb2, respectively. An FRS2 $\alpha$ mutant mouse model, in which the two tyrosine residues that mediate Shp2 binding and the subsequent activation of the Ras/ERK signaling pathway are mutated, shows severe developmental eye defects. In these FRS2 $\alpha$ mutant mice, development of both the neural retina and lens are disrupted, suggesting the Shp2-mediated, Ras/ERK branch of the FGF signaling transduction pathway is required to carry out the inductive FGF signals required for proper lens induction and neural retinal differentiation (Gotoh et al., 2004). In contrast, FRS2 $\alpha$ mutant mice harboring mutations in the four tyrosine residues that mediate Grb2 binding and the subsequent activation of the PI3 kinase/Akt signaling pathway, have no early eye development defects (Gotoh et al., 2004), indicating this arm of the FGF signaling cascade is not involved in the inductive FGF signals directing early eye development. This underscores the complexity that is involved in understanding how FGF signaling can affect early eye development. In addition to the already intricate temporal and spatial expression patterns of individual FGF family members and FGFR isoforms, the activation of various signal transduction pathways and branchpoints must also be considered for their role in coordinating proper ocular development. 


\section{E. Transdifferentiation of retinal pigment epithelium (RPE) to neural retina}

In normal ocular development, the optic vesicle neuroepithelium is composed of retinal progenitor cells that have the potential to take on a neural or non-neural (RPE) retinal cell fate. In some vertebrate species, for a short time after the presumptive neural retina and RPE domains are specified, those presumptive cells still have the capability to reverse their fate and transdifferentiate into the other cell type--the RPE maintains this transdifferentiation capability much longer than cells of the neural retina (reviewed in Lopashov and Stroeva, 1964). Understanding the molecules and pathways that can induce transdifferentiation of RPE to neural retina, as well as the mechanisms that regulate the RPE's potential to transdifferentiate, may provide insight that can be applied to the development of regenerative therapies for the replacement of damaged and diseased neural retinal cells. In terms of this work, RPE-to-neural retinal transdifferentiation provides a mechanism to test whether our gene of interest, the retinal homeobox gene $(R x)$, is absolutely required for neural retinal specification.

\section{Classic studies}

Historically, the transdifferentiation potential of the RPE was demonstrated by surgical manipulations and explant cultures of embryonic eye tissue in various amphibian species. However, many of the amphibian species used have inherent regenerative abilities, which may complicate their applicability towards human therapies. Perhaps more useful, classic studies done in rats, chick and fish, demonstrate embryonic RPE can transdifferentiate into neural retina by: (a) removal of the primary neural retina, (b) release of tension in the RPE by reducing intraocular pressure, (c) removal of the extraocular mesenchyme, or (d) presence of neural retina or some other neural tissues (Coulombre and Coulombre, 1965; Lopashov and Stroeva, 1964). In 
rats, chick, and fish, the RPE can transdifferentiate only during early embryonic stages. More specifically, the RPE's capacity to transdifferentiate into neural retina persists until about E14 in rats and about E4 in the chick (Coulombre and Coulombre, 1965; Lopashov and Stroeva, 1964; Zhao et al., 1997; Zhao et al., 1995). For both embryonic stages, the RPE has already begun differentiating and pigment deposition is evident (Zhao et al., 1997).

\section{$\underline{\text { FGFs and FGF signaling }}$}

As described in detail above, substantial evidence exists from various FGF family members and downstream signaling components that validates the ability of FGFs to induce formation/transdifferentiation of neural retinal cells from RPE both in vitro and in vivo, and across various model species (Guillemot and Cepko, 1992; Hyer et al., 1998; Nguyen and Arnheiter, 2000; Park and Hollenberg, 1989; Park and Hollenberg, 1991; Pittack et al., 1997;

Pittack et al., 1991; Sakaguchi et al., 1997; Spence et al., 2007; Vogel-Hopker et al., 2000; Zhao et al., 2001; Zhao and Overbeek, 1999; Zhao et al., 1997; Zhao et al., 1995). Of particular importance to this dissertation is a RPE-to-neural retina transdifferentiation model caused by transient, ectopic expression of Fgf9 in the dorsal RPE and the lens, the OVE1070 (FGF9) transgenic mouse (Zhao et al., 2001). In this animal model, Fgf9 expression is driven by the lensspecific $\alpha$ A-crystallin promoter, but probably due to genome-integration-site effects, the FGF9 transgene is also ectopically and transiently expressed in the presumptive dorsal RPE from E10.5-11.5, causing cells in this region to adopt a neural retinal fate. This FGF-induced transdifferentiation model, along with the other FGF-induced models, highlights the importance of FGFs in neural retinal differentiation. These studies are further corroborated by experiments in which various elements of the FGF signal transduction cascade are ectopically expressed in the 
RPE and also induce transdifferentiation, providing some of the mechanisms by which FGF directs neural retinal differentiation.

Ectopic expression of two proteins of the Ras/ERK signal transduction cascade within the presumptive RPE is able to drive its transdifferentiation into neural retina. First, in transgenic mouse embryos that ectopically express an activated human $R A S$ oncogene in the presumptive RPE, the RPE is converted into a second layer of neural retina (Zhao et al., 2001). Similarly, ectopic expression of a constitutively activated allele of $M E K-1$, the immediate upstream activator of ERK, in the presumptive RPE of developing chicks induces transdifferentiation of the RPE into neural retina (Galy et al., 2002). Taken together, these results mimic the transdifferentiation induced by FGFs, and indicate activation of the Ras/ERK signaling pathway, a known transduction pathway associated with FGFR activation, is most likely involved in the mechanism by which FGF induces neural retinal differentiation.

Transcription factors and other mechanisms of RPE-to-neural retinal transdifferentiation

One of the hallmarks of FGF-mediated neural retinal transdifferentiation is a rapid downregulation of the bHLHZip transcription factor, microphthalmia-associated transcription factor, (Mitf; Nguyen and Arnheiter, 2000), which has a key function in the development of the RPE (Bharti et al., 2006). A report describing an ERK-dependent mechanism that triggers Mitf degradation in melanocytes provides a potential model to describe how FGFs induce neural retinal differentiation. Briefly, Mitf can be phosphorylated directly by ERK at serine 73, and by p90 Rsk-1, a protein kinase activated by ERK, at serine 409. These phosphorylation events upregulate Mitf's transactivation potential but simultaneously target Mitf for ubiquitin-dependent proteolysis, resulting in rapid degradation and downregulation of Mitf. Thus, applying these 
observations from melanocytes with the results from FGF- and Ras/ERK pathway-mediated transdifferentiation experiments, it is possible that FGF-mediated neural retinal differentiation involves activation of the Ras/Raf/MEK/ERK pathway, which in turn, directly leads to Mitf downregulation. Downregulation of Mitf may be the key component that controls the RPE's ability to switch cell fates. Indeed, embryos homozygous for microphthalmia mutations (nonfunctional Mitf) exhibit transdifferentiation of the RPE to neural retina, suggesting Mitf loss-offunction is sufficient to induce neural retinal formation (Bumsted and Barnstable, 2000; Mochii et al., 1998b; Nakayama et al., 1998; Packer, 1967; Scholtz and Chan, 1987).

The homeobox transcription factor, Chx10 (now known as Vsx2), has been shown to have antagonistic function against Mitf-ectopic expression of Chx10 in the RPE causes downregulation of Mitf and a loss of pigment in the RPE-similar to FGF-induced transdifferentiation models (Horsford et al., 2005; Rowan et al., 2004). Interestingly, Horsford et al. (2004) demonstrate FGF-mediated downregulation of Mitf requires Chx10 activity, indicating FGF and ChxlO are part of a neural retinal differentiation pathway that works by repressing Mitf. Moreover, ectopic expression of Mitf in the presumptive neural retina is able to induce neural retina-to-RPE transdifferentiation in a Chx10-null background, but not in a wild-type background that maintains normal Chxl0 function, confirming Chxl0 represses neuroretinal Mitf transcription to specify and/or maintain neural retinal identity. This is further supported by the expression patterns of Mitf and ChxlO in normal eye development. Mitf is expressed throughout the entire optic vesicle, but following optic vesicle contact with the surface ectoderm, its expression is downregulated in the presumptive neural retina, at the time that $C h x 10$ expression is initiated (Liu et al., 1994; Nguyen and Arnheiter, 2000). 
Supporting a role for Mitf downregulation in RPE-to-neural retinal transdifferentiation, a report published by Baumer et al. (2003) shows Pax6; Pax2 compound mutant mice undergo a dose-dependent reduction of Mitf expression and transdifferentiation of RPE into neural retina. Individually, Pax6 and Pax2 mutants are each able to pattern the optic vesicle into RPE, neural retina, and optic stalk domains, despite the early arrest of Pax6-null optic vesicle development (Baumer et al., 2003; Grindley et al., 1995; Torres et al., 1996), suggesting Pax2 and Pax6 may have redundant function in early optic vesicle patterning. Importantly, both Pax6 and Pax2 single mutants maintain Mitf expression. In vitro studies in this same report show Pax2 and Pax6 can both bind and activate a Mitf RPE promoter, suggesting Pax2 and Pax6 are genetically upstream of Mitf, and that loss of both Pax2 and Pax6 causes RPE-to-neural retina transdifferentiation through the downregulation of Mitf and loss of RPE identity (Baumer et al., 2003).

Intriguingly, a recent report shows that removal of the embryonic chick neural retina at E4 causes spontaneous downregulation of Mitf, but does not induce RPE-to-neural retinal transdifferentiation (Spence et al., 2007). However, exposure of these retinectomized eyes to FGF2 causes activation of the Ras/ERK signal transduction pathway, an increase in Pax6 expression, and transdifferentiation of the RPE to neural retina. Interestingly, the report shows Mitf overexpression, within these retinectomized/FGF2 exposed eyes, inhibits Pax6 expression and prevents transdifferentiation, while having no affect on the Ras/ERK signaling pathway, suggesting Mitf blocks transdifferentiation by directly inhibiting Pax6 rather than inhibiting the Ras/ERK signaling pathway. In support of this mechanism, in vitro and in vivo studies demonstrate Pax6 lies downstream of FGF-ERK signaling (Azuma et al., 2005; Mikkola et al., 1999), and also, Pax6 and Mitf have been shown to inhibit each other's transcriptional activity 
by direct protein-protein interactions in vitro (Mochii et al., 1998a; Planque et al., 2001). While the finding that Mitf can be spontaneously downregulated following retinectomy and fail to undergo transdifferentiation is inconsistent with data from Mitf mutant studies (Bumsted and Barnstable, 2000; Mochii et al., 1998b; Nakayama et al., 1998; Packer, 1967; Scholtz and Chan, 1987), the idea that Pax6 is an important mediator of transdifferentiation is supported by embryonic chick experiments that show in ovo electroporation of human Pax6 alone is sufficient to convert RPE to neural retina, even at later stages (up to chick stage 40, E14)(Azuma et al., 2005). These chick embryo studies conflict with the Baumer et al. (2003) report described above, in terms of how Pax6 and Mitf interact to effect transdifferentiation. However, it is possible that species-dependent differences exist in the mechanisms of transdifferentiation between mouse and chick.

Besides FGF treatment, and some of the signaling cascade components and transcription factors that lie downstream of FGF signaling, a few other genes and signaling pathways have been shown to induce neural retinal transdifferentiation from the presumptive RPE. Similar to Mitf mutants, mice deficient in the Otx genes, Otxl and Otx2, show an inability to assume RPE identity and thus transdifferentiate the presumptive RPE into neural retina. Interestingly, in vitro studies in RPE cells show Otx2 has the ability to bind Mitf and cooperatively activate pigmentation genes, suggesting Otx2 and Mitf may function at the same hierarchical level and cooperate in the activation of genes required for RPE identity (Martinez-Morales et al., 2003).

Growth arrest specific gene 1 (Gas I) encodes a GPI-anchored membrane protein associated with hedgehog (HH) signaling (Martinelli and Fan, 2007). It functions as a cell cycle inhibitor of the $\mathrm{G}(0)$ to $S$ phase transition and is expressed throughout the developing eye. Mice homozygous for a Gas l-null mutation show transdifferentiation of the ventral RPE into neural 
retina (Lee et al., 2001). While it is not entirely clear why Gas 1 mutants only transdifferentiate the ventral RPE into neural retina, this transdifferentiation phenotype suggests that Gasl is essential (in a region-dependent manner) for inhibiting the cell cycle and regulating growth, which is important for the maintenance of an RPE fate.

Sox2 is a high mobility group (HMG) transcription factor that plays important roles in maintaining neural progenitor/stem cell properties and in converting fibroblasts into induced pluripotent stem (iPS) cells. In vivo and in vitro studies done in cells of the developing chick eye show that ectopic Sox2 expression elicits transdifferentiation of RPE into neural retina (Ma et al., 2009). Interestingly, Sox2 and FGF2 expression patterns show significant overlap in normal early eye development as well as atrophic and injured retina models. Furthermore, Sox2 overexpression increased FGF2 expression in the retina and in RPE culture, and reciprocally, Sox2 was induced in RPE cell cultures treated with FGF2. Taken together, Sox2 and FGF2 may be involved in a mutual feedback loop that promotes neural retinal differentiation and is capable of reprogramming RPE into the neural fate.

Another transcription factor that can induce RPE-to-neural retinal transdifferentiation is the neurogenic bHLH factor Mashl (Lanning et al., 2005). Transgenic mice that ectopically express Mash 1 in the presumptive RPE show downregulation of RPE markers and induction of markers characteristic of early neural retinal differentiation, indicating Mashl's ability to convert RPE-fated cells to the neural retinal lineage. Likewise, ectopic expression of the myc oncogene, another transcription factor of the bHLH class, is able to induce transdifferentiation of RPE to the neural retinal fate, but may do so by reprogramming RPE cells to become bipotential retinal progenitors (Beche-Belsot et al., 2001). 
Two recent reports provide evidence that the $\mathrm{Wnt} / \beta$-catenin signaling pathway is important in RPE differentiation via regulation of Mitf and Otx2 (Fujimura et al., 2009; Westenskow et al., 2009). Conditional inactivation of $\beta$-catenin in the presumptive RPE transdifferentiates RPE into neural retina, indicating Wnt/ $\beta$-catenin signaling promotes the RPE fate and inhibits the neural retinal fate. Furthermore, chromatin immunoprecipitation and luciferase assays indicate $\beta$-catenin binds near to and activates potential TCF/LEF sites in the Mitf and Otx2 enhancers, providing a direct mechanism for Wnt/ $\beta$-catenin signaling to promote RPE identity. Interestingly, RPE-restricted expression of a stable form of $\beta$-catenin protein that accumulates in cells and constitutively activates Wnt signaling, disrupts RPE development without causing transdifferentiation, indicating proper RPE development requires an appropriate balance of Wnt signaling (Fujimura et al., 2009).

Bone morphogenetic proteins (BMPs) belong to the TGF- $\beta$ (Transforming Growth Factor- $\beta$ ) superfamily and a recent study shows that inhibition of BMP signaling prevents RPE formation and induces transdifferentiation into neural retina (Muller et al., 2007). Injection of noggin-expressing $\mathrm{CHO}$ cells (noggin binds BMP dimers and prevents their interaction with cell surface receptors) or overexpression of a dominant-negative BMP receptor are able to block BMP signaling and cause the loss of RPE marker expression and induction of neural retinal markers. Conversely, implantation of beads coated with recombinant BMP4 or BMP5 promotes the ectopic formation of RPE in the presumptive neural retina and optic stalk, indicating BMP signaling is sufficient to induce the RPE fate.

Like BMPs, activin is another TGF- $\beta$ family member that plays a role in RPE development. A recent study shows activin signaling regulates the RPE's ability to undergo FGF-mediated transdifferentiation (Sakami et al., 2008). In chick embryos, the ability of RPE to 
generate neural retina in response to FGF2 is lost after stage 25 (Pittack et al., 1991), but a small molecule inhibitor for activin-like kinases, SB431542, is able to extend the RPE's competence to undergo FGF2-mediated transdifferentiation to stage 29. Similarly, activin signaling inhibition by SB431542 in E12 mouse RPE explants enhanced their ability undergo FGF2-mediated transdifferentiation in a dose-dependent manner, suggesting activin, or a related TGF- $\beta$ family member, controls the RPE's capacity to transdifferentiate into neural retina.

Sonic hedgehog (Shh) plays a critical role in establishing the bilateral eye fields and in determining the proximal-distal axis of the eye primordium. But later in eye development, similar to BMP signaling effects, hedgehog (Hh) signaling plays a role in RPE maintenance. Inhibition of Hh signaling, via injection of anti-Shh antibody producing 5E1 hybridoma cells, causes transdifferentiation of the ventral RPE into neural retina (Zhang and Yang, 2001). Similarly, in retinectomized chick eyes exposed to FGF2, inhibition of Hh signaling by KAAD (3-keto, N-amino-ethyl aminocaproyl dihydrocinnamoyl) enhances the transdifferentiation of RPE into neural retina (Spence et al., 2004). Conversely, overexpression of Shh inhibits FGF2mediated transdifferentiation, suggesting Hh signaling functions to maintain RPE identity and inhibit the neural retinal fate.

This review of retinal transdifferentiation provides insights into the complex mechanisms and factors involved in specifying the RPE versus the neural retinal cell fate. In the work included in this dissertation, one model of RPE-to-neural retinal transdifferentiation, the OVE1070 (FGF9) transgenic mouse model, is used to test the importance of $R x$ in this early fate decision in vertebrate eye development. 


\section{F. Markers in early eye development}

Early eye development can be schematically divided into three main morphological events: (a) specification of the eye/retinal field from the anterior neural plate; (b) bilateral evagination of the optic pit/vesicle from this eye field, and (c) formation of the optic cup and lens from the optic vesicle and surface ectoderm, respectively. Each of these steps is regulated by the coordinated activities of growth and transcription factors-some of which still remain unknown. However, the roles of the different known factors can be gleaned from their spatial and temporal expression patterns, from their loss-of-function phenotypes, and from phenotypes caused by overexpression, ectopic expression, and/or gain-of-function mutations. The following genes are known to be critical in these early eye development events:

$\underline{O t x 2}$

Otx2 encodes a homeodomain-containing transcription factor of the bicoid family that is essential for RPE specification. Of the transcription factors important in early eye development, Otx2 is expressed the earliest. It is initially expressed in the epiblast of early mouse embryos at E5.5 (Simeone et al., 1993). Its expression persists in the entire embryonic ectoderm for some time after the onset of gastrulation, but is then progressively restricted to the anterior embryonic ectoderm corresponding to presumptive fore- and midbrain. (Simeone et al., 1992; Simeone et al., 1993). As the optic vesicle evaginates, Otx2 is expressed throughout the optic vesicle, but like Mitf, becomes restricted to the presumptive RPE during optic cup formation, where it is maintained throughout adulthood (Bovolenta et al., 1997; Simeone et al., 2002). At E12.5, as photoreceptor cells begin to differentiate, a second wave of Otx2 expression is found in the neural retina in photoreceptor precursor cells and continues in the outer neuroblastic layer that 
corresponds to the photoreceptor layer of the mature retina (Nishida et al., 2003). In the laminated, postnatal retina, Otx2 is expressed in the inner nuclear layer (Nishida et al., 2003).

Early in development, Otx2 functions to specify forebrain and midbrain, as $O t x 2^{-/-}$mice show a complete lack of all forebrain and midbrain structures, and thus lack the tissue to generate an eye (Matsuo et al., 1995; Pannese et al., 1995). Interestingly, a recent report shows Otx2, along with Sox2, can bind the conserved noncoding sequence (CNS1) 2kb upstream of the $R x$ promoter to activate $R x$ expression, suggesting a mechanism by which $O t x 2$ is coordinated with Sox2 and $R x$ to affect eye formation (Danno et al., 2008).

Studies in Xenopus demonstrate that the domain of Rx expression in the anterior neural plate lies within that of Otx2 (Andreazzoli et al., 1999). Rx is able to repress Otx2 expression in the anterior neural plate to define the retina anlage, and simultaneously position Otx 2 expression for its role in defining the RPE. The eventual restriction of $O t x 2$ expression to the presumptive RPE further suggests a role in specifying the RPE fate. Consistent with this, overexpression of Otx2 in avian neural retinal cells results in pigmentation, one of the hallmarks of RPE identity (Bharti et al., 2006). Furthermore, evidence exists for cooperation with Mitf, another transcription factor important in RPE development, in the binding and activation of pigmentation genes in the developing RPE (Martinez-Morales et al., 2003). Conversely, Otx2-null heterozygotes and $O t x 1^{-/-} ;$Otx $2^{+/-}$compound mutants display decreased expression of RPEspecific genes, such as Mitf and tyrosinase, and transdifferentiate the RPE into neural retina.

Conditional ablation and overexpression studies, targeting the second wave of Otx2 expression, show Otx2 also functions to control photoreceptor cell fate determination of retinal progenitors through the transactivation of the cone-rod homeobox gene, $\operatorname{Crx}$ (Nishida et al., 
2003). Conditional inactivation of Otx2 in differentiating photoreceptor cells caused a fate change to amacrine-like neurons.

\section{$\underline{\operatorname{Six} 3}$}

Six3 encodes a homeodomain-containing transcription factor of the Six/Sine oculis family that shares over $90 \%$ amino acid identity with the Drosophila Six-homeodomain gene, Optix (Toy et al., 1998). The SIX domain, which gives the gene its family grouping amongst other homeodomain proteins, lies upstream of the homeodomain and recruits factors to accomplish transcriptional activation or repression (Granadino et al., 1999; Kawakami et al., 1996) It is first expressed in the developing anterior neural plate around E6.5 in the mouse, and continues its expression in the eye field and throughout the optic vesicle (Bovolenta et al., 1998; Granadino et al., 1999; Loosli et al., 1998; Oliver et al., 1995). As the optic cup begins to form, its expression is restricted to the optic stalk and neural retina. As the neural retina matures (E14.5-E17.5), Six3 is expressed in the inner neuroblastic layer (Zhu et al., 2002). At P0, Six3 expression is found in the inner nuclear and retinal ganglion cell layers. In the adult retina, expression of Six 3 is restricted to amacrine and horizontal cells in the inner nuclear layer and to retinal ganglion cells. Six 3 expression is also found in the developing lens, but there seems to be species differences in its timing (Chow and Lang, 2001). In mouse lens development, Six3 expression first appears during formation of the lens placode, around E9.5 and continues in the lens epithelium. Outside of the eye, Six 3 is expressed in multiple tissues including the ventral diencephalon, olfactory placodes, Rathke's pouch, and the optic stalk (Oliver et al., 1995).

Eighteen different human SIX3 mutations have been identified in multiple cases of holoprosencephly (HPE), a disease defined by an incomplete separation of the cerebral 
hemispheres. While these mutations can cause a wide spectrum of HPE phenotypes, they are often associated with severe brain defects and microphthalmia (Solomon et al., 2009). Likewise, Six3 mutant mouse embryos fail to form the prosencephalon stemming from an inability to repress Wnt signaling and specify the forebrain (Lagutin et al., 2003). These mutations highlight a role for Six3 in the developing anterior neural plate.

Although the timing and pattern of Six 3 expression suggests it has a role in retinal development, the functions of Six 3 in the developing retina remain largely unknown. Ectopic expression in the hindbrain/midbrain regions of developing mouse embryos promotes the formation of optic vesicle-like structures (Lagutin et al., 2001; Lagutin et al., 2003). Inactivation of the medaka fish Six3 with morpholino oligonucleotides results in the absence of forebrain and eyes (Carl et al., 2002). Interestingly, mildly affected embryos exhibit mild cyclopia, small eyes and a reduction of proximal regions of RPE, which mimics the phenotype of human SIX3 mutations and suggests a role in eye patterning along the proximo-distal axis. Overexpression of Six3 results in retinal hyperplasia, suggesting a role in the control of retinal progenitor proliferation. Further, Six3 has also been shown to promote retinal progenitor cell proliferation via interaction with geminin, a negative regulator of cell cycle progression (Del Bene et al., 2004), and promote anterior neural plate proliferation through the activation of cell cycle regulators, cyclinD1 and p27, and anti-neurogenic genes Zic2 and Xhairy2 (Gestri et al., 2005).

Ectopic expression of murine Six 3 in medaka fish is able to induce ectopic lens formation in the otic vesicle in a cell non-autonomous manner, suggesting ectopic expression of Six3 may induce a soluble factor that promotes ectopic lens formation (Oliver et al., 1996). Interestingly, Six3 expression is lost in Pax6 mutant embryos (Sey/Sey), suggesting Six3 is downstream of Pax6 in the genetic pathway for lens formation (Lagutin et al., 2001). 
A mechanism that Six3 uses to exert its various biological functions in eye development involves its interaction with transcriptional co-repressors, Grgs and TLEs, related to the Drosophila Groucho family, to potently repress transcription of its target genes (Kobayashi et al., 2001; Lopez-Rios et al., 2003; Zhu et al., 2002). Interestingly, interaction of Six 3 with the Grgfamily of co-repressors is required for Six 3 transcriptional auto-repression. Further, interactions of Six 3 and Groucho-related co-repressors have developmental implications in the eye. Point mutations in the Grg-binding motifs in Six3 reduce its eye and forebrain enlarging activities, and inhibit its ability to disrupt lens formation in a Six3 overexpression model (Kobayashi et al., 2001; Zhu et al., 2002). Also, interaction of Six3 and TLE1 cause a synergistic expansion of the eye field (Lopez-Rios et al., 2003). Taken together, these examples suggest Groucho-related corepressors are required for Six 3 to perform its functions in eye development.

\section{$\underline{\operatorname{Pax6}}$}

Pax6 is paired box homeobox gene that has maintained an extremely high level of conservation throughout evolution. It encodes a member of the Pax family of transcription factors and contains two DNA-binding motifs: the paired domain and paired-type homeodomain (Walther and Gruss, 1991). Pax6 has been reported to be a key regulator of eye development as it is both essential for eye formation in different organisms as well as sufficient to induce ectopic eyes in flies and frogs upon misexpression (Chow et al., 1999; Halder et al., 1995).

Pax6 is initially expressed in the presumptive forebrain and hindbrain of E8.0 mice (Grindley et al., 1995; Walther and Gruss, 1991). As the optic vesicle grows out of the anterior neural plate, Pax6 is expressed throughout the optic vesicle. In the optic cup, Pax6 is expressed in both the presumptive RPE and neural retinal layers, but later is expressed only in ganglion and 
amacrine cells. In the developing lens, Pax6 expression starts in the surface ectoderm overlying the optic vesicles and is eventually restricted to the proliferating lens epithelial cells (Grindley et al., 1995), and finally in the undifferentiated epithelium in the mature lens (Kammandel et al., 1999). Outside of the eye, Pax6 is essential for normal development in other organs, including the nasal epithelium, brain, pancreas, and lacrimal gland.

The small eye (Sey) mouse mutation is a semidominant Pax6-null allele that causes the small eye phenotype in heterozygous mice (Hill et al., 1991; Hogan et al., 1986) and rats (Matsuo et al., 1993), and is characterized by cataractogenesis, iris hypoplasia, and microphthalmia. Corresponding mutations in human PAX6 are associated with Peters' anomaly (Hanson et al., 1994) and the ocular syndrome, aniridia (Glaser et al., 1992; Hanson et al., 1993; Jordan et al., 1992; Ton et al., 1991). Interestingly, transgenic studies in mice showed that the heterozygous Sey phenotype could be rescued by the introduction of one normal copy of $\operatorname{Pax} 6$, however mice with more than two normal copies of Pax6 also displayed developmental eye defects (Schedl et al., 1996). These studies demonstrate the importance of Pax6 gene dosage.

Similar to its expression pattern in both early optic vesicle and presumptive lens ectoderm, the functions of Pax6 are widespread, underlining its importance in overall eye organogenesis. During early eye development, Sey/Sey mice have abnormal optic vesicles that fail to specify neural retina, and also fail to form a thickened lens placode to initiate lens formation, highlighting the requirement of Pax6 for both retina and lens development (Grindley et al., 1995). This is corroborated by evidence from Pax6 chimeric mice studies (Collinson et al., 2000; Quinn et al., 1996). Pax $^{-{ }^{--}}$cells are excluded from the surface ectoderm and lens placode of chimeric embryos, indicating Pax6 is required in a cell-autonomous manner for lens formation. In chimeric optic vesicles and neural retinas, wild-type and mutant cells segregate and 
mutant cell patches fail to differentiate, demonstrating the cell-autonomous requirement for Pax6 in neural retinal cell differentiation.

To dissect the separate functions of Pax6 in lens and retinal development, studies have been performed that selectively inactivate Pax6 in either the developing lens (Ashery-Padan et al., 2000) or the developing neural retina (Marquardt et al., 2001). First, a Pax6 conditional mutant model was created, "Le-mutant," that inactivated Pax6 specifically in the pre-lens placode surface ectoderm (Ashery-Padan et al., 2000). The specific loss of Pax6 in the pre-lens ectoderm blocks lens placode formation, but in the Le-mutant, optic cup formation is delayed and results in the differentiation of multiple neural retinal folds within the cup. Therefore, Pax6 in the lens seems to be necessary for both lens placode formation and the control of neural retinal development in the optic cup. Conditional inactivation of $\operatorname{Pax} 6$ in the distal neural retina, before onset of cell differentiation, restricts the potential of retinal progenitor cells, limiting Pax6 mutant cell fate to just a subset of non-glycinergic amacrine cells (Marquardt et al., 2001). This indicates Pax6 plays an essential role in maintaining the multipotency and proliferative capacity of retinal progenitor cells. Interestingly, this work also showed that $\operatorname{Pax} 6$ in retinal progenitors is required for the expression of some of the proneural bHLH transcription factors, providing the mechanism that Pax6 uses to promote the formation of other neural retinal cell fates.

Also in early retinal development, $P a x 6$, with its normal expression pattern in the entire optic cup, has been shown to antagonize Pax2 activity in the optic stalk to define the optic stalkoptic cup, proximal-distal boundary (Schwarz et al., 2000) and also direct the determination of RPE (Baumer et al., 2003). Of particular interest to this dissertation, $R x$, through its paired-type homeodomain, can interact with and activate Pax6, providing a mechanism by which Rx can regulate Pax6 and properly coordinate eye development (Mikkola et al., 2001). 
$\underline{\text { Sox2 }}$

Sox2 encodes a transcription factor that belongs to the SoxB1 subfamily of transcription factors characterized by a high mobility group (HMG) domain that is closely related to that of the mammalian sex-determining gene $S R Y$ (Pevny and Placzek, 2005). Sox2 acts as a transcriptional activator with its activation domain located in the C-terminal half. Like many other SOX proteins, Sox 2 has been shown to regulate transcriptional activation through specific interactions with co-factors.

Sox2 is initially expressed throughout the entire ectoderm along the proximal-distal axis of the egg cylinder and then becomes confined to cells that are committed to a neural fate. Thus, Sox2 serves as a universal marker for neural stem and progenitor cells throughout the central nervous system, including the retina. Recently, it has been shown that Sox 2 and neural progenitor identity is regulated by Wnt signaling (Van Raay et al., 2005). As such, Sox2 functions as a key regulator of neural progenitor identity, promoting self-renewal by sustaining the undifferentiated state of progenitor cells and maintaining their ability to either proliferate or differentiate. This is corroborated by its application as one of four genes used to reprogram differentiated cells into induced pluripotent stem cells (Park et al., 2008; Takahashi et al., 2007; Takahashi and Yamanaka, 2006; Yu et al., 2007). Also, as described above, ectopic Sox 2 expression in the RPE is sufficient to reprogram those cells into the neural retinal fate (Ma et al., 2009). Sox2-null mutant mice are embryonic lethal at the preimplantation stage, further demonstrating the importance of Sox2 in very early stages of embryonic development (Avilion et al., 2003).

In the context of early eye development, Sox 2 is expressed in the anterior neural plate as the optic vesicles evaginate from the forebrain, and is subsequently restricted to the neural retina as the optic cup forms (Uchikawa et al., 1999; Uwanogho et al., 1995). In the neural retina, 
similar to other parts of the CNS, Sox 2 expression is maintained in retinal progenitor cells throughout retinal development, and is generally lost as cells differentiate into the post-mitotic neural retinal cell types (Taranova et al., 2006). However, in the mature retina, Sox2 expression remains in Müller glia and a subset of amacrine cells (Le et al., 2002; Lin et al., 2009).

In the developing lens, Sox 2 expression is initiated in the surface ectoderm, dependent on the presence of the optic vesicle. Expression is maintained in the lens placode and lens pit, and then is eventually down-regulated in the lens vesicle and mature lens (Kamachi et al., 1998). Interestingly, Sox2 expression in the surface ectoderm is down-regulated in Sey/Sey embryos, while Sox 2 misexpression in the surface ectoderm is able to up-regulate Pax6, raising the possibility that reciprocal gene regulation by Sox2 and Pax6 might help to restrict and maintain the expression of these genes within the lens placode (Kamachi et al., 2001). Further, Sox2 has been shown to bind cooperatively with Pax6 to the lens $\delta$-crystallin minimal enhancer DC5, and transactivation studies demonstrate Sox2 and Pax6 can act synergistically. Taken together, the transient activity of Sox2 in early lens development is required to initiate the lens program in concert with Pax6.

Mutations in Sox2 are associated with a range of retinal and ocular malformations, such as anophthalmia and microphthalmia (Fantes et al., 2003; Hagstrom et al., 2005; Ragge et al., 2005). Most mutations identified thus far produce hypomorphic conditions, where residual Sox 2 expression and function are still preserved, leading to highly variable severity of the clinical phenotype. A gene-dosage allelic series of Sox 2 mutations in the mouse demonstrate that severity of eye phenotypes correlates to the levels of Sox2 expression found in the neural retinal progenitors (Taranova et al., 2006). Also, retinal progenitors with conditionally ablated Sox2 lose competence to both proliferate and terminally differentiate, demonstrating the importance of 
Sox2 in retinal development and neural retinal cell differentiation. Further, Sox 2 has been shown to directly activate transcription of Notchl, a negative regulator of neural differentiation, thus providing a mechanism for Sox 2 to promote the maintenance of neural retinal progenitor cell identity. Of particular interest to this dissertation, Sox 2 has been shown to physically interact with $O t x 2$ and synergistically activate $R x$ expression through the conserved noncoding sequence (CNS1) located $\sim 2 \mathrm{~kb}$ upstream of the $R x$ promoter (Danno et al., 2008), providing a means by which these factors help coordinate the developmental program of the early eye.

\section{$\underline{\operatorname{Ch} x 10}$}

Chx10 (now called Vsx2) encodes a homeodomain transcription factor of the paired class that also contains a CVC domain. It is initially expressed in the mouse at E9.5 in the distal wall of the optic vesicle that makes contact with the overlying head surface ectoderm (Liu et al., 1994). It is the earliest known transcription factor specifically expressed in the presumptive neural retina following close contact with the surface ectoderm and inductive signaling from the presumptive lens ectoderm (Liu et al., 1994; Nguyen and Arnheiter, 2000). Its expression continues in neural retinal progenitor cells as the optic cup forms, and is terminated as these progenitors become postmitotic and differentiate. As neural retinal differentiation proceeds, Chx10 expression is restricted to the inner nuclear layer, and finally, in the mature retina, ChxlO is exclusively expressed in bipolar cells.

Null mutations in Chx10 cause congenital microphthalmia in humans and in mice (Burmeister et al., 1996; Ferda Percin et al., 2000). The ocular retardation $\left({ }^{J}{ }^{J}\right)$ mouse mutation is a Chx10-null allele caused by a premature stop codon in the homeobox (Burmeister et al., 1996). $o r^{J} / o r^{J}$ mice are blind, with obvious microphthalmia, a cataractous lens, a thin retina, and 
no optic nerve (Truslove, 1962). Characterization of early eye development in $\mathrm{or}^{J} / \mathrm{or}^{J}$ mice indicates $C h x 10$ is required for unimpaired retinal progenitor cell proliferation and bipolar cell differentiation, consistent with its pattern of expression (Burmeister et al., 1996; Liu et al., 1994). In addition, $o r^{J} / r^{J}$ eye development shows the progressive transdifferentiation of neural retinal cells into pigmented cells and a concomitant upregulation of Mitf in these cells, demonstrating Chx10 functions to repress Mitf, specify the neural retinal cell fate, and define the neural retinal domain (Horsford et al., 2005; Rowan et al., 2004). As mentioned above, experiments have shown that FGF-mediated transdifferentiation of RPE to neural retina, and Mitf repression, are dependent on Chx10. Thus, Chx10 is a central player in defining the neural retinal domain from the uncommitted optic vesicle: When the optic vesicle makes contact with the surface ectoderm, FGF from the surface ectoderm directs Chxl0 expression in the distal optic vesicle, which specifies neural retina through the downregulation of Mitf.

The microphthalmia and thin retina seen in $o r^{J} / o r^{J}$ mice are a direct result of reduced proliferation in the absence of Chx10 (Burmeister et al., 1996). Chx10; $p 27^{\text {Kip } 1}$ double null mice are able to rescue the microphthalmic phenotype and proliferation defects in $o r^{J} / o r^{J}$ mice, indicating Chx10 promotes cellular proliferation by negatively regulating the cyclin-dependent kinase inhibitor, $p 27^{\text {Kipl }}$ (Kip1), likely through cyclin D1 activity (Green et al., 2003). Kip1 is expressed in most if not all retinal cell types as they exit the cell cycle during differentiation and homozygous Kip1-null mice have retinal defects as demonstrated by a high level of ectopic RPC proliferation and focal dysplasia (Cunningham et al., 2002; Dyer and Cepko, 2001; Levine et al., 2000; Nakayama et al., 1996). Thus, multiple lines of evidence supports a mechanism in which Chx 10 regulates retinal progenitor cell proliferation by activating cyclin D1 expression, which in turn prevents Kip1 protein accumulation and retinal progenitor cell differentiation. 
Mitf (microphthalmia-associated transcription factor) encodes a basic-helix-loop-helix leucine zipper transcription factor that regulates the differentiation and development of melanocytes and retinal pigment epithelium and is also responsible for pigment cell-specific transcription of genes encoding melanogenic enzymes (Hodgkinson et al., 1993; Shibahara et al., 2001). Heterozygous mutations in the Mitf gene cause auditory-pigmentary syndromes. Mitf consists of at least five isoforms, Mitf-A, -B, C, H, and M, which differ at their N-termini and in their patterns of expression. Mitf-M is restricted to neural crest-derived melanocytes, while MitfA, $-\mathrm{H}$, and $-\mathrm{D}$ are prevalent in the RPE. Mitf-A is the predominant form expressed in the RPE (Amae et al., 1998). Mitf binds to and transactivates target genes carrying the hexameric motif CATGTG (M-box) in their promoter region (Jackson et al., 1991). Those target genes include tyrosinase, the tyrosinase-related proteins, $\operatorname{Trp} 1$ and $\operatorname{Trp} 2$ (also known as Dct, dopachrome tautomerase), which are all enzymes in the melanin pigment synthesis pathway (Smith et al., 1998).

In the developing mouse eye, Mitf is initially expressed in the entire optic vesicle, but is then restricted to the presumptive RPE as the optic cup is formed (Bora et al., 1998; Nguyen and Arnheiter, 2000). The downregulation of Mitf in the presumptive neural retina is coincident with the onset of Chx10, suggesting an antagonistic relationship exists between Mitf and Chx10 in the determination of separate neural retinal and RPE domains. Indeed, experiments using Mitf and Chx10 transgenic and mutant mice indicate an antagonistic interaction between Mitf and Chx10 in determining RPE and neural retinal fates/domains (Horsford et al., 2005). Further, in mice homozygous for the microphthalmia mutation (mi), which is a Mitf-null allele, the dorsal RPE transdifferentiates into neural retina, demonstrating the importance of Mitf in specifying and/or 
maintaining RPE identity (Bumsted and Barnstable, 2000; Mochii et al., 1998b; Nakayama et al., 1998; Packer, 1967; Scholtz and Chan, 1987). Conversely, overexpression of Mitf in cultured avian neural retina or RPE cells induces a pigmented phenotype (Mochii et al., 1998b; Planque et al., 1999).

A complete understanding of how Mitf is regulated remains largely unknown, however a couple of studies offer some insight. In embryonic chick explants, removal of the extraocular mesenchyme covering the optic vesicle prevents RPE formation and blocks the induction and maintenance of Mitf expression (Fuhrmann et al., 2000). Interestingly, both can be rescued by the TGF $\beta$ family member activin, suggesting TGF $\beta$ signaling, emanating from the overlying extraocular mesenchyme, may direct the patterning of RPE through the activation of Mitf. In $\operatorname{Pax6}^{-{ }^{-}} ; \mathrm{Pax}^{-{ }^{--}}$double mutant mice, Mitf expression is absent and the resultant ocular tissue transdifferentiates to neural retina. Furthermore, both Pax2 and Pax6 are able to bind to and activate Mitf in vitro, suggesting Pax2 and/or Pax6 may be important in coordinating Mitf activation and determining the RPE fate.

\section{G. The Retinal Homeobox Gene, $R x$}

\section{History of $R x$ isolation}

The retinal homeobox gene, $R x$ (also called $R a x$ ), encodes a paired-like homeodomain transcription factor that has a critical role in the eye development of several vertebrate species. The $R x$ gene was first identified and reported in three independent studies. In the first study, by Casarosa et al. (1997), $R x$ was isolated from a stage 24/25 Xenopus cDNA library by screening with the murine Orthopedia probe. Simultaneously, Mathers et al. (1997) isolated the Xenopus $R x$ genes from a Xenopus stage-13 cDNA library by screening with homeobox sequences 
amplified from a cDNA library generated from $\mathrm{NH}_{4} \mathrm{Cl}$-induced Xenopus animal caps by PCR with degenerate paired-class primers. This study also identified $R x$ genes in mouse, zebrafish, and Drosophila (Mathers et al., 1997). Similarly, Furukawa et al. (1997) isolated mouse Rx by using degenerate oligonucleotide primers to amplify paired-type homeobox sequences from E18 and $\mathrm{P} 4$ rat retinas, which were then used to screen a P0-P4 mouse retinal cDNA library.

\section{$\underline{\text { Structure of } R x}$}

The coding region of the mouse $R x$ gene is composed of three exons with the homeobox split between exons 2 and 3 (Fig. 3A). Analysis of the mouse $R x$ sequence shows the proposed protein product to have 342 amino acids. However, two putative translation initiation codons are present in the same open reading frame as the homeodomain. The $R x$ gene has four main domains that share similarities with other homeodomain protein components (Burglin, 1994; Furukawa et al., 1997; Mathers et al., 1997): (a) an octapeptide domain spanning amino acid residues 33-40; (b) the homeodomain from amino acids 136-195; (c) a PST-rich domain; and (d) a C-terminal paired tail, or OAR domain from residues 317-331 (Fig. 3B). The octapeptide domain is related to the Drosophila Engrailed gene repressor sequence eh-1, and likely is used to promote transcriptional repression (Smith and Jaynes, 1996). The paired-like homeodomain is a 60 amino acid domain that confers DNA-binding capabilities to $R x$, and is composed of three $\alpha$ helices (Bopp et al., 1986). The two C-terminal helices form a helix-turn-helix structure, through which $R x$ binds DNA. The $9^{\text {th }}$ amino acid residue position in the third helix (homeodomain residue number 50) plays a key role in determining DNA-binding specificity and places the $R x$ gene in the $\mathrm{Q}_{50}$ sub-class of paired-like homeodomain genes, for its glutamine residue at position 50 (Galliot et al., 1999). The DNA sequences recognized by the homeodomain are seven to eight 
nucleotides in length and contain the core TAAT sequence flanked by three or four additional bases (Beebe, 1994). The PST-rich domain is found between the homeodomain and the Cterminal paired tail (OAR domain), and is named for its abundance of proline, serine, and threonine residues, which is similarly found in Pax6 and Octl (Sturm et al., 1988). The Cterminal paired tail, or OAR domain, is a span of 15 amino acids that shares some similarities with other paired-like homeodomain proteins, in which the domain functions as a transactivation domain (Burglin, 1994).

A

$m R x$ locus on

chromosome 18

Exon 1

Exon 2

Exon 3

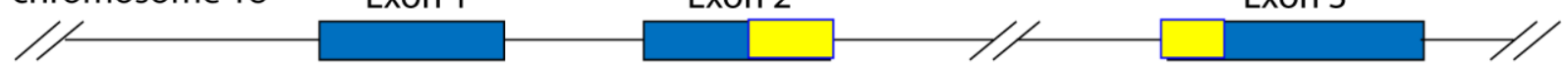

B

Octapeptide (8 aa)

Homeodomain (60 aa)
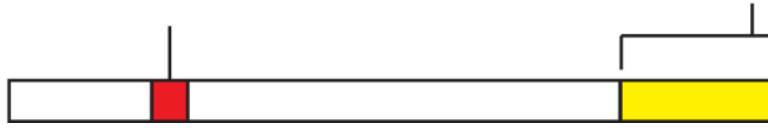

paired tail domain (15 aa)

(also called OAR domain)

PST-rich domain

Figure 3. $R x$ structure.

(A) Organization of mouse $R x$ locus on chromosome 18, homeobox is shown in yellow.

(B) Schematic representation of $\mathrm{Rx}$ protein with domains labeled. 


\section{Expression patterns of $R x$}

The genomes of the mouse, human and fruit fly only contain one $R x$ gene, while other species have multiple homologous $R x$ genes. In chicken, Xenopus, and medaka fish, there are two homologous $R x$ genes, and three in zebrafish. In the mouse, the $R x$ gene is located in the distal region of chromosome 18 (Furukawa et al., 1997). Expression of $R x$ initiates at E7.5 in the neural head fold of developing mouse embryos (Furukawa et al., 2000; Mathers et al., 1997). Expression continues in the anterior neural plate at E8.5, and at E9.0 is restricted to the optic pits and a narrow band of cells in the ventral forebrain. At E9.5, $R x$ expression is found throughout the optic vesicles, the optic stalk and the ventral diencephalon, and by E10.5, as the optic cup forms, its expression is restricted to the presumptive neural retina. Expression in the neural retina is maintained throughout retinal development, but is terminated as cells become postmitotic and terminally differentiate. At postnatal day (P)6, $R x$ expression is detected in the photoreceptor and inner nuclear layers, and in the adult retina, $R x$ is maintained in Müller glial cells (Furukawa et al., 2000). $R x$ expression has also been detected in the ciliary body of adult mouse eyes, an area that contains multipotent retinal progenitor-like cells (Lord-Grignon et al., 2006). Outside of the eye, $R x$ expression is detected in the hypothalamus, posterior pituitary, and the pineal gland.

The general pattern of $R x$ expression in the developing mouse eye is similar to the expression patterns of the other species' Rx gene(s), and together point to conserved roles in anterior neural plate patterning, eye formation, retinal progenitor cell proliferation, and ventral forebrain development across vertebrate species (Bailey et al., 2004). However, some speciesdependent differences in expression pattern exist. In species with multiple copies of $R x$, some have evolved separate functions of $R x$ amongst its multiple copies. Also, there are speciesdependent differences in the cell types that maintain $R x$ expression in the mature retina. Rx 
expression is maintained in Müller glial cells of the adult mouse retina (Furukawa et al., 2000), and in the retinal ciliary margin of mature Xenopus retinas (Mathers et al., 1997). In adult zebrafish retina, expression of $R x 1$ and $R x 2$ is maintained in cones, but not rods, and $R x 3$ is found in the inner nuclear layer (Chuang et al., 1999). Adult medaka express $R x 3$ in the inner nuclear layer (Deschet et al., 1999), and mature chicken retinas express $c$ Rax in the inner nuclear layer (likely Müller glia), and $c R a x L$ in both the inner nuclear layer and in developing photoreceptors.

\section{Functional studies of $R x$}

The first functional studies of the $R x$ gene involved the targeted deletion of $R x$ in the mouse (Mathers et al., 1997). A $R x$-null allele was generated by homologous recombination of a 2.7-kb portion of the mouse $R x$ locus, which included exons 1 and 2, with a $1.8-\mathrm{kb}$ neomycin selection gene. Mice homozygous for the $R x$-null allele have no visible eye structures, while mice heterozygous for the $R x$-null allele are phenotypically normal. Developmentally, $R x^{-/-}$ embryos fail to form optic pits, the neuroectodermal outpouchings from the anterior neural plate that give rise to the optic cups, demonstrating $R x$ is required for eye formation from its initial stages. Interestingly, expression of Six3, Pax6 and Otx2, which are all normally expressed in the anterior neural plate and upregulated in retinal progenitor cells, is maintained in the anterior neural plate of $R x^{-/-}$embryos, but not upregulated, suggesting $R x$ may play a role in the maintenance of retinal progenitor cell identity (Zhang et al., 2000). The failure to form optic vesicles, or any subsequent eye structures, limits the $R x^{-/-}$model's usefulness in assessing $\mathrm{Rx}$ functions in the optic vesicle and later stages of eye development. 
To circumvent this limitation, mouse embryonic chimeras were generated consisting of wild-type and $R x^{-/-}$mutant cells (Medina-Martinez et al., 2009). Analysis of these chimeras show $R x$-deficient cells are excluded from the neural retina, the RPE, and the distal optic stalk, indicating $R x$ is required in a cell-autonomous manner to participate in the formation of these early eye structures and overall, specify the retinal fate. Notably, chimeras made up of a majority of $R x^{-1-}$ cell show a pigmented optic vesicle remnant phenotype, which is very similar to $R x$ conditional inactivation models generated and described in this dissertation. The importance of $R x$ in specifying the retinal fate is further supported by studies that show ectopic expression of $R x$ is able to direct the formation of retinal cells from mouse embryonic stem (ES) cells (Tabata et al., 2004). Interestingly, when cocultured with retinal explants, $R x$-induced ES cells were able to migrate into the host retina and express ganglion and horizontal cell markers, while uninduced ES cells were unable to do either. These results suggest more specialized retinal progenitor cells, as opposed to pluripotent embryonic stem cells, may be able to integrate into the host retina more efficiently, and thus, may be more conducive for use as replacement or regenerative therapies.

Experiments done in Xenopus demonstrate the importance of $R x$ activity for retinal progenitor cell proliferation and maintenance of multipotency (Andreazzoli et al., 1999; Casarosa et al., 2003; Mathers et al., 1997). Overexpression of $R x$ via injection of Xrxl synthetic RNA into 2-8 cell stage Xenopus embryos causes the development of ectopic RPE and hyperproliferation of retinal tissue and neural tube, proliferation of retinal progenitors, and a delay in neuronal differentiation (Andreazzoli et al., 1999; Casarosa et al., 2003; Mathers et al., 1997). A downstream target of $R x$ that may mediate these proliferative effects is the high mobility group B3 gene (Xhmgb3) - its overexpression alone causes increased eye and brain sizes in Xenopus (Terada et al., 2006). Conversely, injection of a Xrxl dominant repressor 
construct consisting of $X r x l$ fused to the repressor domain of Drosophila engrailed (Xrxl-EnR), causes the formation of underdeveloped eyes or, in extreme cases, complete anophthalmia (Andreazzoli et al., 1999). Further, $X r x l$ overexpression in single retinal progenitors via lipofection increases clonal proliferation and no changes in the proportions of the different neural retinal cell types generated, whereas overexpression of cyclin-dependent kinase, $c d k 2$, a strong mitotic promoter, also increases clonal proliferation, but shows a decrease in early-born retinal cell types (ganglion cells and cones) and an increase in late-born types (bipolar cells) (Casarosa et al., 2003; Zaghloul and Moody, 2007). Thus, in addition to promoting proliferation of retinal progenitor cells, $R x$ also functions to maintain retinal progenitor cell multipotency, allowing retinal progenitors to generate postmitotic cells of each of the retinal cell types.

Overexpression of $R x$ in $\mathrm{P} 0$ rat retina, via murine retroviral vector, promotes the formation of Müller glia, through the activation of anti-neurogenic genes, notch1 and Hes1 (Furukawa et al., 2000). Notchl and Hes1, like Rx, are expressed in retinal progenitor cells and are downregulated in differentiating and mature neurons (Bao and Cepko, 1997; Tomita et al., 1996). Similarly, forced expression of either notchl or Hes 1 also promotes the formation of Müller glia, suggesting Rx, notchl and Hes1 are part of a transcriptional cascade that promotes the selection of the glial cell fate. This is interesting in light of the fact that Müller glial cells have been recently characterized as late-stage neuronal progenitors in the retina that function as retinal stem cells (Bernardos et al., 2007). Further, controlled misexpression of $R x$ in immature or in more mature rod photoreceptors by the $\mathrm{Nrl}$ promoter or rhodopsin promoter, respectively, showed $R x$ is able to respecify immature rods to the Müller glial fate, but is unable to change the fate of more mature rods (Matsuda and Cepko, 2007). 
Very elegant studies using in vivo, time-lapse confocal microscopy were performed to track individual retinal progenitor cells undergoing optic vesicle evagination in wild-type and eyeless mutant medaka (Rembold et al., 2006). The medaka eyeless mutation results in defective optic vesicle evagination, and is caused by an intronic insertion in medaka $R x 3$ that leads to transcriptional repression of the locus (Loosli et al., 2001; Winkler et al., 2000). Time-lapse analysis of wild-type, eyeless, and $R x 3$ chimeras show that $R x 3$ is cell-autonomously required for retinal progenitor cells to converge and then migrate to drive optic vesicle evagination from the anterior neural plate (Rembold et al., 2006). Thus, in addition to contributing to the specification of the retinal field during the anterior neural plate stage, $R x$ activity is required for the actual migration of retinal progenitors and the initial steps of eye organogenesis.

\section{$\underline{\text { Mutations in } R x}$}

In addition to the medaka eyeless mutation, other mutant eye models exist that provide insight into the functions of $R x$. Mutations in human $R X$ have been associated with microphthalmia, anophthalmia, coloboma and sclerocornea (Lequeux et al., 2008; London et al., 2009; Voronina et al., 2004). In the first report of human $R X$ mutations associated with disease, a patient with anophthalmia and sclerocornea was identified, who was a compound heterozygote for mutations in $R X$. Characterization of the mutant alleles identified a truncated allele caused by a nonsense mutation at amino acid residue 147, and a missense mutation (R192Q), both within the DNA-binding homeodomain of the RX protein (Voronina et al., 2004). Another report describes a patient with bilateral anophthalmia, who is a compound heterozygote for two different $R X$ mutations, both in exon 3: a frameshifting deletion at residue 222 that introduces a premature stop codon, and a nonsense mutation at amino acid residue 303 (Lequeux et al., 2008). 
Finally, a patient with coloboma has been recently identified as having 2 polymorphisms (E44/D44 and Q294Q) previously described by Voronina et al. (2004), as well as a novel point mutation in exon 1 that causes a missense mutation of arginine to threonine at amino acid position 66.

The eyeless mutation in mouse (eyl) has long been used as model for human anophthalmia, as eyeless mice exhibit severe eye and hypothalamic abnormalities (Chase and Chase, 1941; Tucker et al., 2001). In early eye development of eyeless mice, the optic vesicles emerge normally from the anterior neural tube, but are reduced in size and make poor contact with the surface ectoderm. Molecular analysis of these mice have identified the abnormalities to be due to a missense mutation in $R x$ that removes an alternative start codon (M10L), and reduces $R x$ protein levels (Tucker et al., 2001).

Similar to the medaka eyeless mutants, zebrafish chokh mutants lack eyes and fail to undergo optic vesicle evagination (Kennedy et al., 2004; Loosli et al., 2003). The molecular genetic defects have been identified as nonsense mutations in the homeodomain of zebrafish $R x 3$, which result in a truncated, $R x 3$ - null allele. Interestingly, the Loosli et al. (2003) report shows Pax6 and Six3 expression are unaffected by loss $R x 3$, similar to what is described in $R x^{-/-}$ mouse embryos by Zhang et al. (2000), however the authors interpret these results as meaning forebrain patterning and retinal specification occur normally in the absence of $R x 3$. Also of note, zebrafish chokh mutants show that $R x 3$ is required for normal expression of $R x 1$ and $R x 2$. Remarkably, another report describes two new mutant zebrafish $R x 3$ alleles, $c h k^{s}$ and $c h k^{w}$, which reduce, or altogether prevent, RPE formation, while overall eye morphogenesis and neural retinal development and differentiation are able to proceed (Rojas-Munoz et al., 2005). The $c h k^{s}$ mutation is a nonsense mutation that behaves as a $R x 3$-null allele, and $c h k^{w}$ is a missense 
mutation that behaves as a hypomorph. These mutants indicate $R x 3$ functions to specify RPE identity independent of its roles in eye morphogenesis and neural retinal specification and differentiation.

$\underline{\text { Regulation and downstream targets of } R x}$

A full understanding of how $R x$ is regulated to exert its various functions is still lacking. However, studies in Xenopus embryos indicate $R x$ expression can be activated by chordin, noggin, Hedgehog and Wnt pathways (Casarosa et al., 2003; Rasmussen et al., 2001; Zuber et al., 1999), while neurogenin and retinoic acid represses $R x$ transcription (Andreazzoli et al., 2003). Recently, a 4 kb DNA fragment upstream of the medaka $R x 3$ gene has been used to drive Cre expression in transgenic mice in a spatial and temporal pattern identical to that of endogenous mouse $R x$, suggesting the regulatory elements are conserved between medaka and mouse $R x$, and lie within the $4 \mathrm{~kb}$ of sequence before medaka $R x 3$. Also, regulatory elements in the 5' upstream region of the Xenopus tropicalis and laevis $R x$ genes have been mapped and used to direct gene expression into the developing eyes and ventral hypothalamus (Hirsch et al., 2002; Zhang et al., 2003). Furthermore, in a recent study that compared the 5' upstream regions of $X$. tropicalis and laevis $R x$, a conserved non-coding sequence (CNS1) was identified $\sim 2 \mathrm{~kb}$ upstream of the $R x$ promoter (Danno et al., 2008). CNS1 contains binding sites for both Otx2 and Sox2, which can bind each other and synergistically activate $R x$ expression. Thus, Otx2 and Sox2, via CNS1, can function as direct upstream regulators of $R x$ and aid in the coordination of early eye formation.

While gain- and loss-of-function studies provide correlative evidence of downstream targets that are affected by $R x$ activity, direct downstream targets of $R x$ activity have been 
difficult to identify, mostly due to technical difficulties in expressing $R x$ protein for biochemical analyses and inadequate antibodies against $R x$ for use in biochemical analyses (Pete Mathers, personal communication). However, co-transfection reporter assays have provided evidence of $R x$-mediated transcriptional activation of Hesl and Notchl (Furukawa et al., 2000), and Bmp4 (Voronina et al., unpublished manuscript). Another study has shown that $R x$ binds the CAATTAG sequence of photoreceptor conserved element 1 (PCE-1, also called Ret1) and activates promoter constructs of the arrestin and IRBP (interphotoreceptor retinoid-binding protein) genes (Kimura et al., 2000). The PCE-1/Ret1 sequence element is found in the regulatory regions of many known photoreceptor cell-specific genes and may be used by $R x$ to regulate other photoreceptor genes.

\section{H. Site-specific DNA recombination systems: Cre/loxP and FLP/Frt}

Germline deletion of a particular gene or locus can often lead to embryonic lethality or pleiotropic effects that prevent the analysis of the genetic deletion, i.e. failure to form the cells, tissues, organs, or systems the gene is expressed in. A powerful method to circumvent this problem is through conditional inactivation techniques that use the bacterial (Cre/loxP) and yeast-derived (FLP/Frt) site specific DNA recombination systems (Dymecki, 1996; Gu et al., 1993; Shibata et al., 1997; Vooijs et al., 1998). These systems allow spatial and temporal control

of gene inactivation in vivo. These systems are based on the ability of Cre and FLP recombinases to catalyze the excision of DNA flanked by loxP or Frt recognition sequences (O'Gorman et al., 1991; Sauer and Henderson, 1988).

Cre-mediated loxP recombination is well characterized (Abremski et al., 1983; Hoess and Abremski, 1985; Sternberg and Hamilton, 1981). Briefly, a single 38-kDa protein, Cre 
recombinase (encoded by the coliphage P1) is both necessary and sufficient to catalyze recombination between two loxP sites, each of which is $34 \mathrm{bp}$ in length. Cre-mediated recombination is dependent on the orientation of the $\operatorname{lox} P$ sites. LoxP sites oriented in the same direction on the molecule catalyzes the excision of the intervening DNA segment in the presence of Cre recombinase, while inverted loxP sites triggers the inversion of the intervening DNA segment. Similarly, FLP-mediated recombination is catalyzed by Flippase (FLP) from Saccharomyces cerevisiae (O'Gorman et al., 1991). Its recognition sequences are Frt (FLP recognition target) sites, which are also $34 \mathrm{bp}$ in length, and excision of the intervening DNA sequence requires the Frt sites to be oriented in the same direction.

Thus to generate a conditional mutant mouse, first, loxP/Frt sites must be inserted around one or more exons of the target gene via homologous recombination in embryonic stem cells, creating a "floxed" or "flrted" mouse. Then, a conditional mutant mouse is generated by crossing the floxed/flrted mouse to a transgenic mouse expressing Cre or FLP recombinase under the control of a tissue- or cell-specific promoter, which will recombine the LoxP/Frt sites and excise the DNA segment between them.

\section{$\underline{R x \text { conditional alleles }}$}

The inability to form optic vesicles in the $R x^{-/-}$mouse model prevents its use for studying $R x$ function in later stages of eye development. Thus, both Cre/loxP and FLP/Frt DNA recombination systems have been utilized in our lab to generate a series of conditional alleles that allow for the activation and inactivation of $R x$ (Voronina et al., 2005). In particular, the $R x^{\text {flox }}$ allele, and its use in combination with two Cre-expressing animals to generate $R x$ conditional inactivation models, plays a central role in the work included in this dissertation. 
The allelic series at the $R x$ locus was first generated by creating the $R x^{\text {neo }}$ allele through homologous recombination and ES cell technology (Voronina et al., 2005). The $R x^{\text {neo }}$ allele contains a Neomycin-resistance (Neo) cassette in intron 1 that is flanked by Frt sites, and has loxP sites flanking the 5'-Frt site and the 3'-end of exon 2 (Fig. 4A). Insertion of the Neo cassette renders this allele inactive, as $R x^{\text {neo/neo }}$ mice phenocopy $R x^{-/-}$mice in the inability to form optic vesicles during embryogenesis and perinatal lethality. The $R x^{\text {flox }}$ allele was generated by crossing $R x^{\text {Neo }}$ heterozygotes to $\beta$-actin-FLPe, which excises the Neo cassette. $R x^{\text {flox }}$ is so named because exon 2 is still "floxed," and is a functionally active $R x$ allele (Fig. 4B). Thus, $R x^{\text {flox }}$ can be used in conjunction with Cre-expressing strains for $R x$ conditional inactivation strategies, as described in the work for this dissertation. Finally, the $R x^{\Delta 2}$ allele is generated by crossing either a $R x^{\text {neo }}$, or $R x^{\text {flox }}$ heterozygote to a $\beta$-actin-Cre animal, creating an inactive $R x$ allele (Fig. 4C). The $R x^{\Delta 2}$ allele encodes a protein that lacks the homeodomain due to excision of exon 2, and exon 3 is out of frame with exon 1, creating a non-functional, truncated protein product. Indeed, $R x^{\Delta 2 / \Delta 2}$ embryos also phenocopy $R x^{-/-}$embryos, in that they are unable to form optic vesicles and die soon after birth. 
A

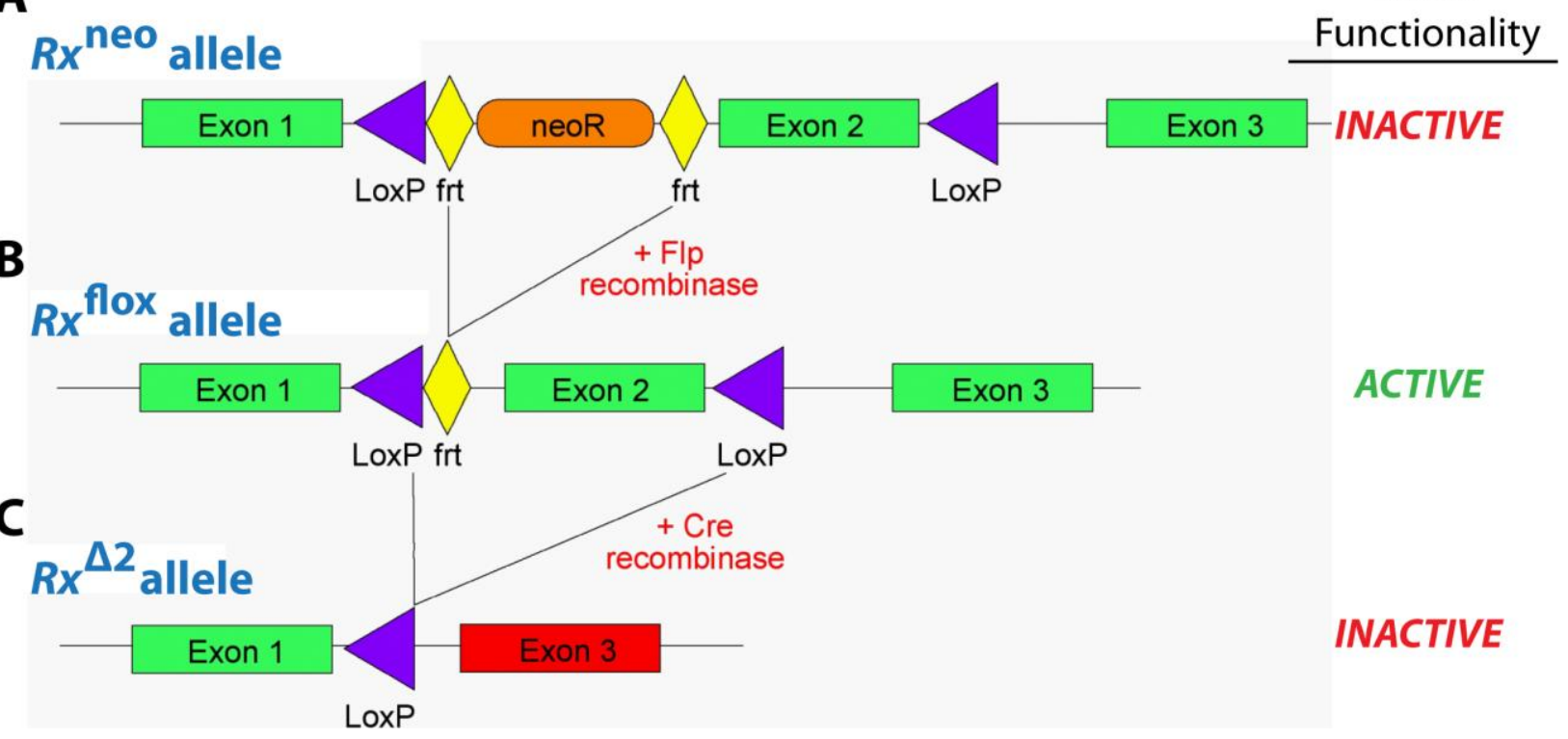

Figure 4. Conditional $R x$ alleles.

(A) $R x^{\text {neo }}$ is an inactive allele due to the insertion of a neomycin resistance (neoR) cassette in intron 1.

(B) $R x^{\text {flox }}$ is an active allele created by FLP-mediated excision of the neoR cassette from the $R x^{\text {neo }}$ allele. Exon 2 is flanked by loxP sites.

(C) $R x^{\wedge 2}$ is an inactive allele created by Cre-mediated excision of exon 2, which causes exon 3 to be out of frame with exon 1 and creates a non-functional, truncated protein product.

\section{$\underline{\text { Foxg1-Cre; } R x \text { conditional mutant }}$}

In the first use of the $R x^{\text {flox }}$ allele, the Foxg1-Cre mouse strain was used to conditionally inactivate $R x$ during the optic vesicle stage of early eye development, generating Foxg1-Cre; $R x$ conditional mutants (Voronina et al., unpublished manuscript). Controlled by the Forkhead box G1 gene locus, Foxg1 (formerly known as brain factor 1, BF-1), Cre is expressed in the telencephalon and in discrete head structures, including the anterior half of the optic vesicle, in Foxg1-Cre animals (Hebert and McConnell, 2000). Thus, Foxg1-Cre-mediated $R x$ inactivation results in the disruption of optic vesicle patterning and proliferation (Voronina et al., unpublished manuscript). Foxg1-Cre; $R x$ conditional mutants arrest eye growth and development during the 
optic vesicle stage and the optic vesicle remnant adopts an RPE fate. Interestingly, expression of some of the secreted factors involved in lens induction-Bmp4, Fgf8, and Fgf15—are downregulated in these conditional mutants, and lens induction does not occur. These results indicate $R x$ is required during optic vesicle development for neural retinal specification and lens induction. In this dissertation, this $R x$ conditional mutant model is crossed to a FGF9-induced, RPE-to-neural retina transdifferentiation model to assess whether $R x$ is absolutely required for neural retinal specification. We specifically test whether FGF signaling can rescue neural retina formation in the Foxg1-Cre; $R x$ conditional mutant. 


\section{Chapter II:}

$R x$ is required for specification of the neural retina, independent of optic cup morphogenesis and lens induction 


\section{ABSTRACT}

Patterning of bipotential optic vesicle cells into distinct regions of RPE and neural retina requires the coordinated activities of both extrinsic signaling factors and intrinsic transcription factors. $R x$ is a paired-like homeobox gene that encodes a transcription factor that is expressed in retinal progenitor cells of the developing optic vesicle/cup. $R x$ expression is lost as retinal progenitors exit the cell cycle and terminally differentiate into a neural retinal cell type, but is maintained through adulthood in Müller glia. During embryogenesis, mice homozygous for a targeted Rxnull allele fail to form optic vesicles, demonstrating $R x$ is intrinsically required for the earliest stage of eye formation - evagination of the optic vesicles. Using a Six3-Cre/loxP model of $R x$ inactivation, we have conditionally inactivated $R x$ in the presumptive neural retinal cells of the developing optic vesicle/cup to address the functions of $R x$ in the early stages of eye development following optic vesicle evagination. Here we show cells that undergo Cre-mediated $R x$ inactivation in the developing optic vesicle/cup exhibit reduced proliferation, fail to express markers of neural retina, and assume an RPE fate, indicating $R x$ is required for retinal progenitor cell proliferation and neural retinal specification. Interestingly, our data supports a model where the proliferative effects of $R x$ have downstream effects on optic cup morphogenesis and lens induction. However, despite these effects on developing eye morphology, our results show that $R x$ is intrinsically required for neural retinal specification amongst the bipotential cells of the optic vesicle, independent of optic cup formation and lens induction.

\section{KEYWORDS}

$R x$, retina, specification, patterning, Six3-Cre, conditional mutant, mouse

\section{INTRODUCTION}

The neural retina, retinal pigment epithelium (RPE), and optic stalk originate from the same sheet of anterior neuroectoderm that evaginates to form the optic vesicle. It is during this stage that critical fate determination decisions are made, impacting early vertebrate eye development - bipotential retinal progenitor cells of the optic vesicle become specified for either neural retinal or RPE fate. Vertebrate eye morphogenesis begins with the bilateral evagination of optic pits from the anterior neuroectoderm, which in the mouse occurs at embryonic day (E)8.5. As each optic pit grows laterally toward the surface ectoderm, the optic vesicle forms. The distal tip of the optic vesicle contacts the lateral head surface ectoderm at E9.5, and once contact is established, inductive signals from the optic vesicle cause thickening of the overlying surface 
ectoderm. Subsequently, the surface ectoderm forms the lens placode, which later invaginates to form the lens vesicle. Concurrently, following close contact with the surface ectoderm, the distal optic vesicle thickens and invaginates to form the optic cup, with the inner and outer layers of the cup ultimately forming the neural retina and RPE, respectively.

It has been generally established that extrinsic signals emanating from surrounding tissues patterns the optic vesicle into three specific domains — dorsal, distal, and ventral—that will give rise to the RPE, neural retina, and optic stalk, respectively. These inductive signaling cues confer tissue identity through the coordinated activation of specific transcription factors (reviewed in Adler and Canto-Soler, 2007). Sonic hedgehog (Shh), secreted from the ventral midline, directs the activation of transcription factors that establish optic stalk identity (Zhang and Yang, 2001), while FGFs from the surface ectoderm (Hyer et al., 1998; Nguyen and Arnheiter, 2000; Pittack et al., 1997) and TGF- $\beta$ signals from the extraocular mesenchyme (Fuhrmann et al., 2000) direct the patterning of the neural retinal and RPE domains, respectively, within the optic vesicle (reviewed by Chow and Lang, 2001).

Substantial evidence points to fibroblast growth factors (FGFs) being an important extrinsic cue from the surface ectoderm that can direct neural retinal specification within the distal optic vesicle. First, FGF1 and FGF2 are abundantly expressed in the presumptive lens ectoderm, and FGF receptors are correspondingly expressed in the optic vesicle (de Iongh and McAvoy, 1993; Miller et al., 2000; Nguyen and Arnheiter, 2000; Pittack et al., 1997; Tcheng et al., 1994; Wanaka et al., 1991). Explant studies in mice and chick show that removal of the surface ectoderm prevents neural retinal specification in the distal optic vesicle, which can be rescued by exogenous application or expression of FGFs (Hyer et al., 1998; Nguyen and Arnheiter, 2000). Optic vesicle culture studies in chick show that blocking FGF2 with a 
neutralizing antibody blocks neural retinal development without affecting RPE differentiation (Pittack et al., 1997), and studies in rodents, frog and chick have shown that exogenous application or ectopic expression of FGFs can induce transdifferentiation of the presumptive RPE into neural retina, establishing the importance of FGFs in specifying the neural retinal fate (Guillemot and Cepko, 1992; Opas and Dziak, 1994; Park and Hollenberg, 1989; Pittack et al., 1991; Reh et al., 1991; Spence et al., 2007; Vogel-Hopker et al., 2000; Zhao et al., 2001; Zhao and Overbeek, 1999; Zhao et al., 1995). These lines of evidence indicate FGFs emanating from the surface ectoderm may be required for establishing neural retinal identity in the distal optic vesicle. Similarly, inductive signals emanating from the extraocular mesenchyme are required for RPE patterning in the apposed dorsal optic vesicle domain (Fuhrmann et al., 2000). Removal of the extraocular mesenchyme from chick optic vesicle explants prevents RPE formation in the dorsal optic vesicle, and promotes neuroretinal identity. While the exact molecule(s) exerting this effect remain unidentified, treatment with Activin A is sufficient to rescue RPE formation in optic vesicle cultures with the extraocular mesenchyme removed, suggesting an activin, or some other TGF- $\beta$ family member, provides the inductive signal that directs RPE fate specification.

In addition to the extrinsic factors required for optic vesicle patterning, the intrinsic activities of specific transcription factors are also necessary for patterning the optic vesicle into its neural retinal and RPE domains. Following close contact of the distal optic vesicle with the surface ectoderm and induction by FGFs, Chx10 (or Vsx2) expression is turned on in the distal optic vesicle and antagonizes Mitf to specify the neural retinal domain (Nguyen and Arnheiter, 2000). Similarly, mesenchyme-derived signals lead to the upregulation of Mitf and Otx2 in the dorsal optic vesicle. Mitf antagonizes Chxl0 to pattern the RPE domain and also cooperates with Otx2 to activate melanin pigment biosynthesis genes and initiate the RPE specification program 
(Bovolenta et al., 1997; Hodgkinson et al., 1993; Horsford et al., 2005; Martinez-Morales et al., 2003; Rowan et al., 2004).

In addition to roles in tissue fate specification, many transcription factors also promote the proliferation of retinal progenitor cells for proper eye development. Studies in Xenopus have identified a battery of eye field transcription factors (EFTFs), including ET, Rx1, Pax6, Six3, Lhx2, tll and Optx2 (also known as Six6), that are involved in the specification of the eye field via their coordinated expression in the anterior neural plate (Zuber et al., 2003). Several of these genes have continued expression in retinal progenitor cells and are involved in the further specification of specific retinal cell types (Casarosa et al., 2003; Grindley et al., 1995; Oliver et al., 1995; Walther and Gruss, 1991). Sox2 is a marker for neural progenitors that interacts with Otx2 to synergistically activate $R x$ expression (Danno et al., 2008), positioning them as potential regulators of neural retinal specification in addition to promoting proliferation. Chx10, Pax6, and Six3 are all markers for neural retinal progenitors that drive proliferation and play important roles in the determination of retinal fates (Bovolenta et al., 1998; Burmeister et al., 1996; Liu et al., 1994; Loosli et al., 1999; Marquardt et al., 2001). While advancements have identified transcription factors and interactions involved in retinal development, gaps still exist in our complete understanding of how these genes coordinate the specification and morphogenesis of the neural retina.

$R x$ encodes a paired-like homeodomain transcription factor that is conserved amongst vertebrates and invertebrates. In early vertebrate eye development, $R x$ is initially expressed in the anterior neural plate and the entire developing optic vesicle, and is then restricted to the neural retina during the optic cup stage of development. Within the developing optic cup, $R x$ is expressed in neural retinal progenitors until they terminally differentiate, leading to the 
hypothesis that $R x$ plays a role in the proliferation of retinal progenitor cells (Casarosa et al., 2003; Furukawa et al., 1997; Furukawa et al., 2000; Mathers et al., 1997). In the adult retina, $R x$ expression remains present in Müller glial cells (Furukawa et al., 2000), which can function as retinal stem cells (Bernardos et al., 2007). Mutations in $R x$ across several model species cause developmental eye defects, demonstrating the importance of $R x$ in early vertebrate eye formation (Kennedy et al., 2004; Loosli et al., 2003; Loosli et al., 2001; Tucker et al., 2001). Mutations in human $R X(R A X)$ are associated with microphthalmia, anophthalmia, coloboma, and sclerocornea (Lequeux et al., 2008; London et al., 2009; Voronina et al., 2004), and targeted homozygous deletion of murine $R x\left(R x^{--}\right)$results in complete anophthalmia by preventing the earliest stage of eye formation - evagination of the optic pits from the neural ectoderm (Mathers et al., 1997). The homozygous $R x$-null mouse model demonstrates the requirement of $R x$ for initiating eye morphogenesis, but because subsequent stages of eye development never ensue, this deletion model cannot be used to explore $R x$ functions beyond optic pit evagination.

In this work, we make use of a Cre/loxP conditional inactivation strategy (Voronina et al., 2005) to ascertain $R x$ 's function in early eye development beyond the initiation of optic pit formation, in particular, its role in retinal progenitor cell proliferation and neural retinal specification. We report that conditional inactivation of $R x$ in the developing optic vesicle/cup, using the Six3-Cre transgenic mouse model (Furuta et al., 2000), allows for optic vesicle formation, and even optic cup formation in some cases, but causes a marked reduction in proliferation, a failure to express neural retinal markers, and causes the mislocalized expression of RPE markers in the distal optic vesicle/prospective neural retinal domain. In addition, despite the initiation of optic cup morphogenesis and lens induction, we show that $R x$ is required for specification of neural retinal identity in bipotential retinal progenitor cells of the optic vesicle. 


\section{Materials and Methods:}

Genetic cross for $\boldsymbol{R} \boldsymbol{x}$ inactivation in the developing optic cup. Six3-Cre transgenic mice (strain \#69, kindly provided by Dr. Guillermo Oliver, St Jude’s Research Hospital, Memphis, TN) were crossed with mice from our $R x$-null strain to create Six3-Cre; $R x^{+/-}$males. The Six3-Cre and $R x$-null strains are as described previously (Furuta et al., 2000; Mathers et al., 1997). Conditional deletion of $R x$ in the developing optic cup beginning at E9.0-9.5 was achieved by crossing Six3-Cre; $R x^{+/-}$males to $R x^{\text {flox/flox }}$, ROSA26-EGFP ${ }^{\text {flox/flox }}$ females. The $R x^{\text {flox }}$ allele has exon 2 flanked by LoxP sites (Voronina et al., 2005), while the ROSA26-EGFP flox/flox allele has a stop cassette, located between the ROSA26 promoter and an EGFP reporter gene, flanked by LoxP sites (Mao et al., 2001). This cross produces the conditional mutant genotype, Six3-Cre; $R x^{\text {flox/- }}$, Rosa26-EGFP ${ }^{\text {flox/+ }}$ (Six3-Cre; $R x$ conditional mutant), in $25 \%$ of the progeny. The control littermates analyzed were Six3-Cre; $R x^{\text {flox/++}}$; Rosa26-EGFP flox/+ mice, which are phenotypically normal and allow for EGFP reporter analysis of the Six3-Cre transgene.

PCR genotyping. Tail biopsies were collected and digested in a PCR-competent lysis solution (50 mM KCL, 10 mM Tris $\mathrm{HCl} \mathrm{pH}$ 8.3, 2 mM $\mathrm{MgCl}_{2}$, 0.45\% IGEPAL CA-630, 0.45\% Tween-20) plus proteinase $\mathrm{K}(0.6 \mathrm{mg} / \mathrm{mL})$ overnight at $55^{\circ} \mathrm{C}$, to extract purified genomic DNA from embryos and pups. PCR reactions were done using the Cre and $R x$ oligonucleotide primers and conditions previously described (Holzenberger et al., 2000; Voronina et al., 2005).

Morphologic and immunohistologic analyses. Embryonic heads were fixed in a 1x PBS solution containing $4 \%$ paraformaldehyde overnight at $4^{\circ} \mathrm{C}$, cryoprotected in $30 \%$ sucrose in PBS, and cryosectioned coronally at $12 \mu \mathrm{m}$. Developing ocular morphology was analyzed by Nuclear Fast Red (Vector Laboratories) staining or DIC microscopy. Immunohistochemistry (IHC) was performed on cryosections. First, epitope unmasking was performed by incubating 
cyrosections in $0.1 \mathrm{M}$ Tris- $\mathrm{HCl}, \mathrm{pH} 9.5$ at $95^{\circ} \mathrm{C}$, for $20 \mathrm{~min}$. The sections were treated with $2 \%$ hydrogen peroxide, blocked for 90 min in normal serum, incubated overnight with primary antibodies at $4{ }^{\circ} \mathrm{C}$, washed with PBS, incubated for $2 \mathrm{~h}$ at room temperature with the secondary antibodies, and washed in PBS. Immunostaining was visualized using the ABC Elite kit (Vector Labs) and diaminobenzidine staining. Primary antibodies were GFP (1:1000, Molecular Probes), activated-Caspase-3 (1:600, Promega), Chx10 (1:50-200, ExAlpha), Six3 (1:500, gift from Oliver lab), Mitf (1:1000, gift from Arnheiter lab), Otx2 (1:1000, Chemicon), Sox2 (1:500, Chemicon), Pax6 (1:50, Developmental Studies Hybridoma Bank, Univ. of Iowa), proliferating cell nuclear antigen (PCNA, 1:1000, Dako), and phosphorylated histone H3 (pHH3, 1:500, Upstate).

Retinal proliferation marker assays. E10.5-E12.5 Six3-Cre; $R x$ conditional mutant eyes were subdivided into two groups representing the most extreme examples on the phenotypic spectrum — severe and mild phenotypes — for comparison against a littermate control group. We categorized our conditional mutant groups based on (a) the degree of optic cup formation and (b) progression of lens development. Mild phenotypes form an optic cup and a lens vesicle, while severe phenotypes show no morphologic signs of optic cup invagination or lens placode formation. Because of the limited prevalence of each extreme phenotype, we also included some moderately affected eyes that were morphologically similar to each of our two groups. At least 3 independent animals were sampled for each group and were cryosectioned coronally through the orbit at $12 \square \mathrm{m}$. To broadly compare proliferation between the two groups, we processed every fifth section through the embryonic eye, per animal, with an anti-PCNA primary antibody (Dako) and an Alexa 647-conjugated donkey anti-mouse IgG secondary antibody (Molecular Probes) for immunofluorescent analysis. 
For mitotic cell counts, cryosections were co-labeled with a mitotic phase marker, phosphorylated histone $\mathrm{H} 3$ (pHH3), and a nuclear stain, propidium iodide (PI, Molecular Probes). Secondary antibody was Alexa 488-conjugated donkey anti-rabbit IgG (Molecular Probes). For each animal ( $\mathrm{n} \geq 3$ ), at least 3 , non-adjacent, central retinal sections were selected for analysis. Within the presumptive eye tissue of each section, the ratio of pHH3-positive cells to PI-stained cells, from the two different Six3-Cre; $R x$ conditional mutant groups, was calculated and compared against the ratios within the RPE and neural retinas of littermate controls. Total retinal (PI-stained) cell counts were compared between each conditional mutant group versus control (neural retina + RPE). Statistical significance of each conditional mutant group's mitotic ratio and total retinal cell count versus control was calculated by one-tailed, unpaired Student's t-test. Fluorescent images were taken with a Zeiss LSM 510 Meta confocal microscope.

\section{RESULTS}

\section{Conditional inactivation of $\boldsymbol{R} x$ during the optic cup stage of eye development causes postnatal anophthalmia.}

To address the functions of $R x$ in the early stages of eye development following optic vesicle evagination, we used the $R x^{\text {flox }}$ allele (Voronina et al., 2005), in conjunction with the Six3-Cre transgenic mouse strain (Furuta et al., 2000), to conditionally inactivate $R x$ in the developing optic cup. The Six3-Cre line expresses Cre recombinase in the developing optic cup and ventral optic stalk, with its onset spanning embryonic day (E)9.0-9.5 (Furuta et al., 2000), during which the optic cup begins to form from the optic vesicle. However, in our hands, the Six3-Cre line gave a slightly broader onset window of Cre recombinase activity, with inconsistent Rosa26-EGFP reporter activity evident even at E9.5 (Supplemental Fig. S1). The 


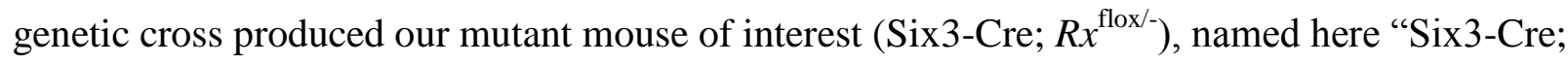
$R x$ conditional mutant" or "conditional mutant," at the expected Mendelian ratio. The control littermates analyzed have the Six $3-\mathrm{Cre} ; R x^{\text {flox/+ }}$ genotype and are phenotypically wild-type. Unlike the $R x$ germline deletion model $\left(R x^{-1}\right)$, Six3-Cre; $R x$ conditional mutants are viable, but share the anophthalmic phenotype (Fig. 1B; Mathers et al., 1997). Dissection of postnatal day $(\mathrm{P}) 21$ conditional mutants revealed only a pigmented cystic remnant present within the orbits (Fig. 1C -D). Careful dissection of P22 brains showed a lack of optic nerves and optic chiasms in the forebrain floor of Six3-Cre; $R x$ conditional mutants (Fig 1F).

\section{Six3-Cre-mediated inactivation of $\boldsymbol{R} x$ during the optic cup stage creates a spectrum of embryonic eye phenotypes.}

To understand how $R x$ inactivation during the optic cup stage of eye development can lead to the postnatal anophthalmic phenotype, we collected embryos soon after Six3-Cremediated $R x$ recombination/inactivation and looked for aberrations in early eye development as compared to control littermates. Grossly, whole-mount E11.5 Six3-Cre; $R x$ conditional mutants show retarded eye growth in comparison to their control littermates (Fig. 2A-D). Upon further histological analysis of these conditional mutants, we were able to distinguish three Six3-Cre; $R x$ conditional mutant eye phenotypes: severely, moderately, and mildly affected eyes. Phenotypic classifications were based on (a) the degree of cupping of the leading/distal-most edge of the optic vesicle, and (b) the extent of presumptive lens development. The severely affected conditional mutant eye phenotype is characterized by no optic cup and no lens placode development (Fig. 2E). The moderately affected eye phenotype has some cupping of the distal optic vesicle (arrow in Fig. 2F) and thickening of the surface ectoderm adjacent to this cupping 
(LP in Fig. 2F), suggestive of lens placode formation. The mildly affected Six3-Cre; $R x$ conditional mutant eye displays greater cupping of the optic vesicle (arrow in Fig. 2G) and formation of a presumptive lens vesicle within this optic cup (LV in Fig. 2G). Notably, we observed litters that contained conditional mutant embryos with different eye phenotypes, and occasionally found individual embryos that had differentially affected eyes (data not shown). Also, both Nuclear Fast Red staining (PG in Fig. 2E-G) and differential interference contrast (DIC) microscopy (PG in Fig. 4J-K), reveal pigment deposition throughout the lumen of the persistent optic vesicles/cups of all Six3-Cre $R x$ conditional mutant phenotypes, suggestive of RPE formation at the distal-most edge of the optic vesicle/cup, where neural retina would normally form.

These phenotypic differences in presumptive eye development are apparent during the earlier stages of embryonic eye development, starting around E10.5. By E14.5, the three different presumptive eye phenotypes are no longer as distinguishable, with the resulting conditional mutant optic tissue resembling nothing more than a persistent optic vesicle/cup and lens tissue no longer detectable (Figs. 3M-N). Interestingly, in the Six3-Cre; $R x$ conditional mutants, we observe a progressive loss of contact between the optic vesicle/cup remnant and the surface ectoderm, and the infiltration of head mesenchymal cells between the two structures, where the lens vesicle would normally develop (asterisks in Fig. 3G-N).

Using optic cup and lens development as phenotypic classification criteria, the distribution of phenotypes amongst E10.5-12.5 Six3-Cre; $R x$ conditional mutant eyes was calculated. The majority of conditional mutant eyes fall into the moderately and severely affected phenotype categories: 51\% (90/176) moderately affected; 39\% (68/176) severely 
affected. The mildly affected Six3-Cre conditional mutant eye phenotype was observed less frequently than the other two at a rate of $\sim 10 \%(18 / 176)$.

\section{$R \boldsymbol{x}$-depleted cells survive, but undergo fate change.}

To follow the fate of $R x$-depleted cells, we used EGFP expression from the ROSA26EGFP $^{\text {flox/flox }}$ reporter allele (Mao et al., 2001) within the Six3-Cre; $R x$ conditional deletion strain to mark cells that had undergone Cre-mediated recombination. Immunolabeling of E10.5-E14.5 Six3-Cre; $R x$ conditional mutants with an anti-GFP antibody showed consistent staining in the resultant optic tissue, regardless of phenotype severity (Fig. 3). Wild-type embryos from E10.5 to E14.5 all show GFP immunolabeling in the optic cup and optic stalk (Fig. 3C, F, I, L, O). E10.5 and E11.5 Six3-Cre; $R x$ conditional mutants show GFP immunolabeling in the presumptive optic cup and ventral optic stalk (arrows in Fig. 3A-E). From E12.5 and beyond, GFP-positive cells populate the distal portion of the persistent optic vesicle/cup that results in the Six3-Cre; $R x$ conditional mutants (arrows in Fig. 3G-N). Despite clear changes in early eye development structure and morphology, the EGFP reporter marks cells in the persistent optic vesicle/cup, regardless of phenotype severity, indicating cells of the $R x$-inactivation lineage remain present in the resultant optic vesicle/cup remnants of Six3-Cre; $R x$ conditional mutants. To test whether changes in ocular morphology could be due to cell death, immunofluorescent staining was performed with an anti-activated Caspase- 3 antibody on conditional mutant cryosections through the eye. No anti-activated Caspase-3 staining was observed throughout the persistent optic vesicles/cups of E10.5-11.5, Six3-Cre; $R x$ conditional mutants, regardless of phenotype severity and (Supplemental Fig. S2). These data demonstrate that $R x$-depleted cells 
persist in the optic tissue remnants without undergoing cell death, and instead undergo a fate change from that of neural retina.

\section{Proliferation is reduced in Six3-Cre; $R x$ conditional mutants.}

Given the proposed role for $R x$ in retinal progenitor cell formation and maintenance (Casarosa et al., 2003; Furukawa et al., 1997; Mathers et al., 1997; Zhang et al., 2000), we examined whether there is a reduction in proliferation for the Six $3-\mathrm{Cre} ; R x$ conditional mutants as a possible explanation for the microphthalmic phenotype observed in the early stages of embryonic eye development. To simplify our analyses, we assayed proliferation in only the two extreme groups on the conditional mutant eye phenotype spectrum: severely affected and mildly affected eyes (see Methods). First, to assess proliferation from a qualitative standpoint, we immunofluorescently labeled E10.5-E12.5 coronal eye sections from Six3-Cre; $R x$ conditional mutants and littermate controls, with an anti-PCNA (proliferating cell nuclear antigen) antibody. PCNA antibody is a widely used marker that labels proliferating cells in all phases of the cell cycle and whose expression is down-regulated in the retina following cell cycle exit and terminal differentiation (Barton and Levine, 2008).

Anti-PCNA immunolabeling in E10.5-E12.5 controls shows robust signal throughout the embryonic sections, with particularly strong labeling in the optic cup/neural retina and lens vesicle — highly proliferative tissues at these early stages (Fig. 4C, F, I). At E10.5 and E11.5 ( 1 and 2 days after Six3-Cre-mediated $R x^{\text {flox }}$ recombination, respectively), Six3-Cre; $R x$ conditional mutants maintain some PCNA-positive staining within the persistent optic vesicle/cup cells that make up the presumptive retina (Fig. 4A-E). By E12.5, conditional mutants are virtually devoid of PCNA-positive, proliferating cells within the presumptive retina (Fig. 4G, H). As a broad 
marker of cellular proliferation, these anti-PCNA immunostaining data show the general trend of reduced proliferation within the developing retina, over time, following the conditional inactivation of $R x$.

To quantify these changes in proliferation, we sought to compare phosphorylated histone H3 (pHH3) immunolabeling between Six3-Cre; $R x$ conditional mutant eyes and littermate control eyes following Six3-Cre-mediated $R x^{\text {flox }}$ recombination. Phosphorylated histone H3 is a marker of cells specifically in the mitotic phase of the cell cycle and is another established indicator of retinal cell proliferation (Barton and Levine, 2008; Prigent and Dimitrov, 2003). We used an anti-pHH3 antibody in conjunction with the nuclear counterstain, propidium iodide (PI), to label and count mitotic cells in relation to total cells within the developing retinal tissue of E10.5-E11.5 Six3-Cre; $R x$ conditional mutants for comparison against littermate control RPE and neural retinal tissue (Fig. 5E-J).

At E10.5, the percentage of cells that were $\mathrm{pHH} 3$-positive in control RPE and neural retina were $2.0 \%( \pm 0.4 \%$ s.e.m.) and $3.6 \%( \pm 0.7 \%$ s.e.m.), respectively. Littermate Six3-Cre; $R x$ conditional mutant eyes had pHH3-positive cell values somewhat comparable to those for control RPE: severely affected eyes, $1.4 \%( \pm 0.4 \%$ s.e.m.; $p$-value $=0.15)$; mildly affected eyes, 1.3\% ( $\pm 0.4 \%$ s.e.m.; $p=0.04$; Fig. $5 \mathrm{~A}$ ). At E11.5, control RPE and neural retina had pHH3positive cell values of $2.4 \%$ ( $\pm 0.4 \%$ s.e.m.) and $4.5 \%$ ( $\pm 0.5 \%$ s.e.m.), respectively, while values for Six 3-Cre; $R x$ conditional mutant eyes significantly dropped in comparison to even control RPE: $0.4 \%\left( \pm 0.1 \%\right.$ s.e.m.; $\left.p=2.6 \times 10^{-4}\right)$ for E11.5 severely affected eyes and $0.9 \%( \pm 0.3 \%$ s.e.m.; $p=2.2 \times 10^{-3}$ ) for E11.5 mildly affected eyes (Fig. 5B). Essentially, one day post-Six3Cre-mediated recombination, mitotic ratios in conditional mutants are comparable to control RPE rates, but are significantly reduced by E11.5. 
Counts of total cells, as judged by PI staining, within the optic tissue of E10.5 and E11.5 conditional mutants and controls reveal a significant reduction in the number of presumptive retinal cells, per central retinal section, in Six3-Cre; $R x$ conditional mutants versus littermate controls $\left(p<10^{-5}\right)$. E10.5 conditional mutants had $191 \pm 15$ and $260 \pm 36$ presumptive retinal cells, per section, for severely $(n=3)$ and mildly $(n=3)$ affected mutants, respectively, as opposed to $547 \pm 31$ retinal cells total $(134 \pm 6 \mathrm{RPE}+413 \pm 28$ neural retina) for the littermate controls $(\mathrm{n}=4$; Fig. 5C). At 11.5, 2 days post-Six3-Cre-mediated $R x^{\text {flox }}$ recombination, conditional mutant eyes were composed of $175 \pm 8$ and $190 \pm 12$ total retinal cells, per section, for the severe $(n=4)$ and mild $(\mathrm{n}=3)$ phenotype groups, respectively, while control eyes $(\mathrm{n}=3)$ had $723 \pm 50$ retinal cells total, per section $(150 \pm 7 \mathrm{RPE}+573 \pm 45$ neural retina; Fig. 5D). These total cell count data suggest that, following Six3-Cre-mediated $R x^{\text {flox }}$ recombination, proliferation of retinal progenitors is stunted in the absence of $R x$ activity. Altogether, these proliferation marker studies, along with the absence of apoptosis at these stages, support the notion that conditional inactivation of $R x$ during optic cup development leads to a decrease in proliferation soon after Cre-mediated recombination. This provides a potential mechanism that could lead to the microphthalmic developmental phenotype observed in Six3-Cre; $R x$ conditional mutant embryos and the eventual anophthalmic phenotype observed in postnatal conditional mutants.

\section{Six3-Cre-mediated inactivation of $\boldsymbol{R} x$ prevents neural retinal specification}

In order to understand the role that the $R x$ gene plays in tissue fate decisions during early embryonic eye development, we performed immunohistochemical marker studies to ascertain the identity of cells in the resultant optic tissue in Six3-Cre; $R x$ conditional mutants. The apparent cupping/thickening of the distal optic vesicle in moderately and mildly affected conditional 
mutant eyes at E10.5 and E11.5 (Fig. 2 and 3) raises the possibility that, despite a reduction in overall presumptive eye size, neural retinal cells may still be specified in the conditional mutants. To test this hypothesis, we performed immunohistochemical staining with anti-Chx10, anti-Six3, and anti-Sox 2 antibodies on each of the three different phenotype classes of Six3-Cre; $R x$ conditional mutants (Fig. 6 and 7). These three transcription factors are known to be expressed in early neural retinal progenitors (Bovolenta et al., 1998; Burmeister et al., 1996; Kamachi et al., 1998; Liu et al., 1994; Loosli et al., 1998; Oliver et al., 1995). Anti-Chx10,-Six3, and -Sox2 antibodies immunolabel neural retinal cells in control sections (arrowheads in Fig. 6D, H, and 7 D), whereas no staining is detected throughout the persistent optic vesicle/cup cells of Six3-Cre; $R x$ conditional mutants, regardless of phenotype (Fig. 6A-G, 7A-C). In particular, the moderately and mildly affected embryos show cupping of the distal optic vesicle (and even some thickening of the optic cup in the mild phenotype), a morphological sign suggestive of neural retinal formation. However, the expression of Chx10, Six 3 , or Sox 2 is never detected in the distal, cupped region of the persistent optic vesicle (Fig. 6B,C,F,G, and 7B,C). Regardless of phenotype severity or degree of cupping, Six3-Cre; $R x$ conditional mutants fail to specify neural retinal tissue within their resultant optic vesicle remnant.

Morphologic analyses of moderately and mildly affected Six3-Cre; $R x$ conditional mutants are suggestive of lens placode and lens vesicle development, respectively, so we checked for immunoreactivity against early lens development markers, Sox2, Pax6, and Six3 (Grindley et al., 1995; Kamachi et al., 1995; Kamachi et al., 1998; Kamachi et al., 2001; Oliver et al., 1995). These lens markers are also expressed in the developing neural retina (see Fig. 6E$\mathrm{H}$ and arrowheads in 7D), as well as RPE for Pax6 (arrowheads in Fig. 7H). In embryos across several stages, all three markers labeled the lens vesicle of control eyes (arrows in Fig. 6H, 7D, 
H). Littermate Six3-Cre; $R x$ conditional mutants of the moderately and mildly affected classes show immunopositive staining for Sox2, Pax6, and Six3 in the surface ectoderm adjacent to the distal optic vesicle remnant (arrows in Fig. 7B-C, F-G, and 6F-G, respectively). Immunostaining with these early lens development markers supports the morphological data that suggest the lens placode is induced in the moderate phenotype, and lens vesicle is formed in the mildly affected Six3-Cre; $R x$ conditional mutants. Developmental progression of the lens in the Six3-Cre; $R x$ conditional mutants indicates that the failure to specify neural retinal fate in the mutant optic vesicle is not due to defects in lens induction.

\section{Six3-Cre-mediated inactivation of $R x$ results in an all-RPE-fated persistent optic vesicle/cup.}

In the persistent optic vesicles/cups of Six3-Cre; $R x$ conditional mutants, regardless of phenotype severity, pigment deposition is observed throughout, including the distal-most region that is normally fated to become neural retina (see Fig. 2E-G, 4J-K). To test whether the resulting pigmented tissue in the conditional mutant is RPE, we performed immunohistochemical staining against two RPE markers, Mitf and Otx2 (Bora et al., 1998; Bovolenta et al., 1997; Hodgkinson et al., 1993; Nakayama et al., 1998; Nguyen and Arnheiter, 2000; Simeone et al., 1993). Both proteins are transcription factors expressed in the presumptive RPE, and have been shown to interact with each other to transactivate the promoters of genes involved in melanin pigment biosynthesis (Martinez-Morales et al., 2003). Staining with anti-Mitf and anti-Otx2 antibodies is specifically observed in the RPE of littermate controls (arrowheads in Fig 8D, H). Consistent with the observations of pigment deposition, both anti-Mitf and anti-Otx2 antibodies stain the entire persistent optic vesicles/cups of Six3-Cre; $R x$ conditional mutants, independent of 
phenotype severity (Fig. 8A-C, E-G). Thus, these markers identify the resultant pigmented tissue as RPE. Interestingly, despite the formation of an optic cup and the initiation of lens placode/vesicle development in the moderately and mildly affected phenotypes, all cells of the persistent optic vesicle take on a RPE cell fate following Six3-Cre-mediated inactivation of $R x$.

\section{DISCUSSION}

Our data from these Six3-Cre-mediated, $R x$ conditional inactivation studies demonstrate $R x$ is necessary for promoting proper retinal progenitor cell proliferation and highlight its requirement for neural retinal specification. The Six3-Cre; $R x$ conditional mutant model, which conditionally inactivates $R x$ during optic cup development, bypasses the $R x$-dependent optic pit stage observed in the germline $R x$ knockout (Mathers et al., 1997), and underscores the importance of $R x$ in the subsequent stages of early eye development.

Our Six3-Cre; $R x$ conditional mutant model shares striking developmental similarities with the classic eyeless inbred mouse strain, ZRDCT (Chase and Chase, 1941), which contains a mutation in an alternative translation initiation site and effectively reduces the abundance of $\mathrm{Rx}$ protein (Tucker et al., 2001). In 90\% of eyeless neonates, there are no eyes or optic tracts, and pups are anophthalmic, while the remaining $10 \%$ end up microphthalmic. During early eye development, the optic vesicle forms normally in eyeless embryos and makes contact with the surface ectoderm (Chase and Chase, 1941). Soon after contact is made between the optic vesicle and surface ectoderm, eyeless embryonic eyes begin to deviate from normal development. Silver and Hughes (1974) contend that a major factor in the eyeless mutant phenotype is the inability of intervening mesenchymal tissue, caught between the optic vesicle/cup and the surface ectoderm, to undergo cell death. These persisting mesenchymal cells then disrupt the inductive signaling 
between the optic vesicle and surface ectoderm and inhibit proper retinal and lens growth and development (Silver and Hughes, 1974). This model is supported by studies that show the capability of head mesenchyme to disrupt ocular morphogenesis and promote RPE fate in the absence of presumptive lens ectoderm (Fuhrmann et al., 2000; Furuta and Hogan, 1998; Nguyen and Arnheiter, 2000).

The Six3-Cre; $R x$ conditional mutant optic vesicle forms normally up to the time contact is made between the optic vesicle and surface ectoderm (Fig. 3). And like the ZRDCT eyeless strain, the conditional $R x$ mutant has intervening head mesenchyme cells between the optic vesicle/cup and surface ectoderm (asterisks in Fig. 3), possibly impeding the reciprocal inductive signals required for the proper development of both neural retina and lens. However, the invasion of mesenchymal cells in the conditional mutants appears to be a secondary effect, as the proliferation defects observed in the severely affected optic vesicle precede the invasion (Fig. 4 and 5). Furthermore, the mildly affected embryos continue to maintain contact between the lens vesicle and the optic vesicle until at least E12.5 (Fig. 4H). Therefore, the disruption in neural retinal specification seen in the Six3-Cre; $R x$ conditional mutant is likely the direct result of $R x$ inactivation and independent of defects in lens or head mesenchyme signaling.

The developmental outcome of Six3-Cre; $R x$ conditional mutant eyes is also similar to that of a recently reported chimeric $R x$ mutant model, where in the presence of mostly $R x$-mutant cells, the entire optic vesicle/cup develops into RPE, and the lens is not induced (MedinaMartinez et al., 2009). Indeed, the optic vesicles/cups of Six3-Cre; $R x$ conditional mutants adopt an RPE fate, and in the case of our most severely affected embryos, the lens tissue is not induced. Interestingly, $R x$ chimeras show $R x^{-/-}$mutant cells are excluded from the neural retina, corroborating our conclusion that $R x$ is required for the specification of neural retina. Both 
models highlight the requirement of $R x$ within the optic vesicle for adequate proliferation to support proper ocular morphogenesis and for the specification of neural retinal identity.

The variability in the onset of Six3-Cre expression, as monitored by Rosa26-EGFP activation (Supplemental Fig. S1; Furuta et al., 2000), is the most likely reason for the variability in developing eye size and the persistent optic vesicle/cup phenotypes observed in E10.5-14.5 Six3-Cre; $R x$ conditional mutants (Fig. 9). Given the importance of $R x$ in the proliferation of retinal progenitor cells (Fig. 4 and 5), we envision a mechanism where early Six3-Cre-mediated inactivation of $R x$ leads to a subsequent drop off in optic vesicle proliferation and growth, yielding the severely affected conditional mutant phenotype (Fig 2A, E, and Fig. 9). Conversely, the mild Six 3-Cre; $R x$ conditional mutant phenotype is likely a result of delayed Cre activation and $R x$ inactivation, where retinal proliferation and growth are affected only after the optic cup has already formed (Fig. 2C, G and Fig. 9). In a similar manner, the timing of $R x$ inactivation leads to progressive changes in the degree of lens induction (Fig. 2, 7, and 9). Although our ROSA-EGFP reporter assays suggest very efficient Six3-Cre-mediated recombination (Fig. 3), we cannot rule out the possibility of mosaic Six3-Cre expression causing the spectrum of early embryonic eye phenotypes in the conditional mutant. However, given that our conditional deletion model requires only a single recombination event to inactivate $R x$, the possibility of mosaicism due to inefficient recombination is unlikely. We also cannot rule out the possibility of variable levels of residual $\mathrm{Rx}$ protein following $R x^{\text {flox }}$ recombination as the cause of the variable phenotypes.

Overexpression studies in Xenopus embryos and mouse pups point to a role for $R x$ in the proliferation of retinal progenitor cells. Overexpression of $X r x l$ can induce hyperproliferation of neural retina and RPE by prolonging the expression of cell cycle markers, including cyclin D1, 
and by inhibiting Xngrl and p27Xic (Andreazzoli et al., 1999; Andreazzoli et al., 2003; Casarosa et al., 2003; Mathers et al., 1997). Further, Terada et al. (2006) have identified a nucleosome regulator, $X h m g b 3$, that is downstream of $R x$ and can affect eye size by promoting proliferation of retinal progenitor cells. In postnatal rats, $R x$ overexpression activates Hes 1 and notchl, two transcription factors that promote retinal progenitor proliferation (Bao and Cepko, 1997; Furukawa et al., 2000; Tomita et al., 1996). However, the studies presented here are the first to show proliferation defects when $R x$ is inactivated in the mammalian retina. Our studies reveal a progressive decrease in proliferating cells upon $R x^{\text {flox }}$ recombination in comparison to the surrounding tissues, as well as a decrease in overall developing eye size (Fig. 2, 4 and 5). The difference is most dramatic at the E11.5 stage, where mitotic ratios for conditional mutants show a greater than $60 \%$ decrease in comparison to mitotic ratios from control RPE (Fig. 5B).

Six3, Chx10, and Sox2 all promote retinal progenitor cell proliferation (Del Bene et al., 2004; Gestri et al., 2005; Green et al., 2003; Taranova et al., 2006). Our immunohistochemical data show that expression of all three of these retinal transcription factors is lost in the persistent optic vesicles of Six3-Cre; $R x$ conditional mutants (Fig. 6 and 7). Thus, it is possible that the decrease in proliferation observed in the conditional mutants is also impacted by the loss of Six3, Chx10, and Sox2, in addition to any direct effects that $R x$ has on proliferation. Interestingly, Six3, Chx10, and $R x$ all affect retinal progenitor proliferation via p27 regulation (Andreazzoli et al., 2003; Gestri et al., 2005; Green et al., 2003).

Hyer et al. (2003) found that optic cup morphogenesis from the optic vesicle neuroepithelium requires a temporally specific association with the presumptive lens ectoderm. Our data corroborate this model and demonstrate the temporal importance of $R x$ for optic cup morphogenesis. The optic cup fails to form in severely affected Six3-Cre; $R x$ conditional mutants 
(Fig. 2 and 3), which could result from proliferation defects that prevent the optic vesicle from making sufficient contact with the presumptive lens ectoderm to promote optic cup morphogenesis. On the other hand, moderately affected conditional mutant optic vesicles may proliferate long enough to allow for the cupping of the distal optic vesicle, yet neural retinal specification is prevented in the absence of $R x$ (Fig. 6). Thus, our data suggest that the effects of $R x$ inactivation on retinal progenitor cell proliferation (i.e. -optic cup morphogenesis and lens placode/vesicle development) are completely independent from its effects on neural retinal specification. Alternatively, studies in medaka have shown that $R \times 3$ is cell-autonomously required for retinal progenitor migration during optic vesicle evagination from the anterior neural plate (Rembold et al., 2006). Thus, it is possible that defects in optic cup morphogenesis reflect a progressive loss of $R x$-mediated cellular migration following Six3-Cre-mediated conditional inactivation of $R x$.

Despite the range of morphological differences in optic cup formation/thickening and lens development amongst Six3-Cre; $R x$ conditional mutants, specification of the optic vesicle remains uniform - the neural retinal cell fate is never specified, and the resulting optic vesicle/cup becomes RPE (Fig. 6, 7 and 8). In this study, our moderate and mild conditional mutant phenotypes demonstrate the intrinsic requirement of $R x$ within the optic vesicle to specify neural retinal identity independent of lens placode/vesicle induction. While early signaling between the optic vesicle/cup and the surface ectoderm allow for the initiation of lens development in the moderately and mildly affected conditional mutants (Fig. 7), the reciprocal signals necessary for proper neural retinal patterning are somehow inhibited. We speculate that neural retinal patterning of the distal optic vesicle could be lost because $R x$ is downstream of 
FGF signaling, and the absence of $R x$ within the optic vesicle/cup blocks the propagation of FGF signaling initiated by the surface ectoderm (Hyer et al., 1998; Zhao et al., 2001).

Our neural retinal marker studies suggest Rx lies upstream of and is required for the expression of Chx10, Six3, and Sox2 in the genetic pathway for neural retinal determination (Fig. 6 and 7). Interestingly, Pax6 expression remains unaffected in Six 3-Cre; $R x$ conditional mutants (Fig. 7), suggesting retinal expression of Pax6 is not controlled by Rx. However, it is more likely that this persistent Pax6 expression reflects specification of an RPE fate throughout the persistent optic vesicle/cup of conditional mutant embryos.

Our data shows both Mitf- and Otx2-positive staining throughout the persistent optic vesicles/cups of conditional mutants, confirming their RPE identity (Fig. 8). Baumer and colleagues (2003) report the requirement of the redundant activities of Pax6 and Pax2 in the specification of RPE — in particular, Mitf activation. Thus, Pax6 expression throughout the persistent optic vesicles/cups of conditional mutants may contribute to the observed pattern of Mitf expression and pigment deposition in developing conditional mutant optic vesicles/cups, and may actually be indicative of Pax6's role in RPE specification (Fig. 7E-G). In addition, reports show that Chx10 represses Mitf for the maintenance of neural retinal identity and defining the neural retinal domain (Horsford et al., 2005; Rowan et al., 2004). Thus, it is possible that the RPE phenotype of the Six3-Cre; $R x$ conditional mutant is due to a lack of Chx10 expression, which could allow Mitf expression to take over and promote the formation of RPE where it would normally be repressed by Chx10. Our Chx10 and Mitf immunolabeling data supports this model of RPE determination in the conditional mutant.

Based on the data presented here, we envision a model wherein the inactivation of $R x$ causes retinal progenitors to exit the cell cycle and prematurely differentiate. At this early stage 
of development, intrinsic factors within the optic vesicle and extrinsic factors in the surrounding tissue drive a differentiation program that favors an RPE fate. Overall, our data show that, during the optic vesicle/cup stage of development, $R x$ plays a critical role in the proliferation of retinal progenitors, which has effects in the overall morphology of the developing eye, and is a factor that is intrinsically required within the optic vesicle for specification and patterning of the neural retina. 


\section{ACKNOWLEDGEMENTS}

We would like to thank Dr. Guillermo Oliver for the Six3-Cre transgenic mouse strain and antiSix3 antibody; Drs. Visvanathan Ramamurthy and Heinz Arnheiter for the gifts of reagents; and Dennis Cole for expert technical assistance. The Pax6 monoclonal antibody developed by A.

Kawakami was obtained from the Developmental Studies Hybridoma Bank developed under the auspices of the NICHD and maintained by The University of Iowa, Department of Biology, Iowa City, IA. This research was supported by EY012152. 

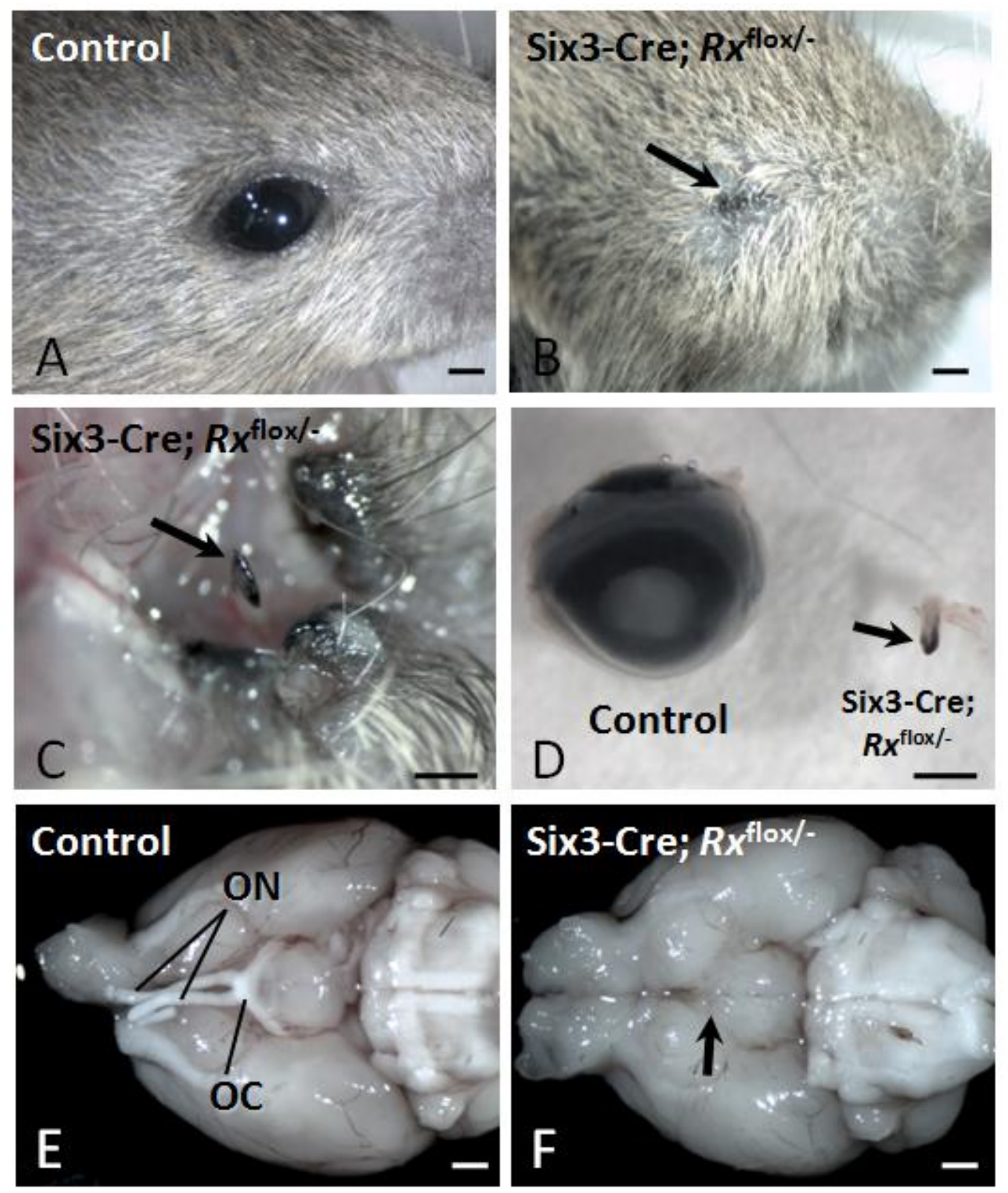

Figure 1. Six3-Cre-mediated conditional inactivation of $R x$ during the optic cup stage of development causes postnatal anophthalmia with a pigmented cystic remnant in the orbit and concomitant loss of optic nerves and optic chiasm. (A and B) Whole-mount P21 orbits from Six3-Cre; $R x$ conditional mutant (Six3-Cre; $R x^{\text {flox/- }}$ B) and control (A). (C) Dissection of Six3-Cre; $R x$ conditional mutant orbit from panel (B). (D) Enucleated ocular tissue from P21 Six3-Cre; $R x$ conditional mutant and control. Note pigment deposition on the distal tip of the cystic remnant (arrow in D). (E and F) Whole-mount $\mathrm{P} 22$ brains from control $(\mathrm{E})$ and Six3-Cre; $R x$ conditional mutant $(\mathrm{F})$ animals. Note the lack of optic nerves $(\mathrm{ON})$ and optic chiasm $(\mathrm{OC})$ in the conditional mutant brain (arrow in F). Scale bars: $1 \mathrm{~mm}$. 


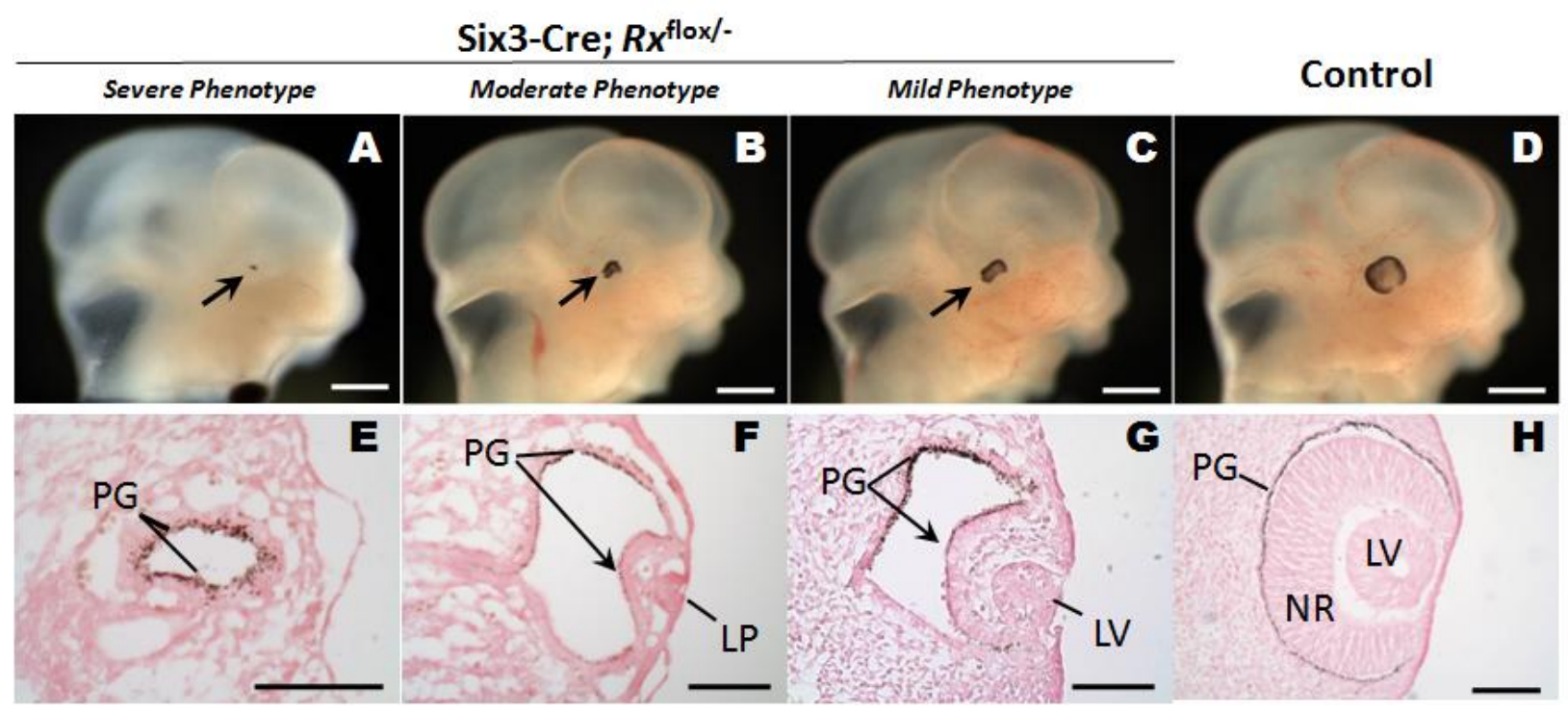

Figure 2. Six3-Cre-mediated inactivation of $R x$ in the optic cup creates a range of early eye developmental phenotypes spanning severely to mildly affected eyes. (A-D) Wholemount E11.5 heads of Six3-Cre; $R x$ conditional mutants (A-C) and control (D). All three iterations of the conditional mutant phenotype have an optic rudiment (arrows in A-C) that is much smaller than that of their control counterpart (D). (E-H) Nuclear Fast Redstained coronal sections through the orbit of E11.5 Six3-Cre; $R x$ conditional mutants (E$\mathrm{G}$ ) and control littermate $(\mathrm{H})$. Note the pigment granules (PG) deposited throughout the lumen of the persistent optic vesicles in the conditional mutants. The severe conditional mutant phenotype $(E)$ is composed of pigmented tissue with no lens placode or optic cup formation. The moderate conditional mutant phenotype shows some cupping of the optic vesicle (arrow in F) and thickening of the surface ectoderm, suggestive of lens placode formation (LP). The mild conditional mutant phenotype features greater cupping of the optic vesicle (arrow in $G$ ) and formation of a presumptive lens vesicle within the optic cup (LV). PG, pigment granules; LP, lens placode; LV, lens vesicle; NR, neural retina. Scale bars: A-D, $500 \mu \mathrm{m}$; E-H, $100 \mu \mathrm{m}$. 


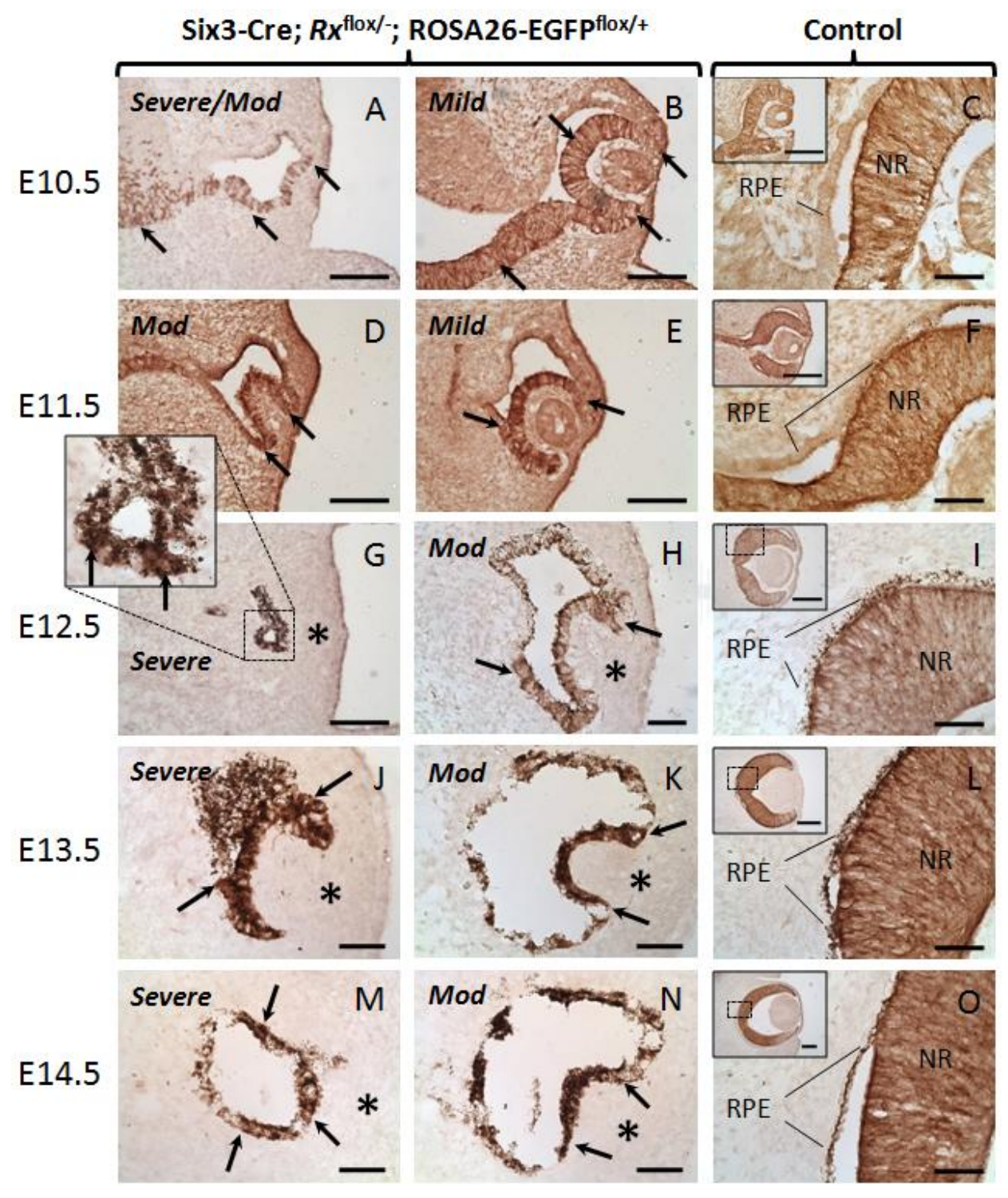

Figure 3. Fate change for Six3-Cre; $R x$ conditional mutant cells. Cells that have undergone Six3-Cre-mediated recombination continue to populate the optic remnants of $R x$ conditional mutants, indicating that upon inactivation of $R x$, EGFP-positive cells originally fated for the optic cup and the neural retina, as shown in the controls, remain within the mutant optic vesicles. (A-O) EGFP reporter activity assayed by immunohistochemistry with an anti-GFP antibody on coronal sections through the orbit at the corresponding stages in Six3-Cre; $R x$ conditional mutants and littermate controls. The EGFP reporter is activated in the control optic cup throughout early eye development (C, F, I, L, and O); insets show whole sections of the embryonic eye. Arrows in panels point to GFP-positive tissue. Asterisks indicate infiltration of head mesenchymal tissue in conditional mutants (G-O). RPE, retinal pigment epithelium. Scale bars: C, F, H-O, $50 \mu \mathrm{m}$; A, B, D, E and G, $100 \mu \mathrm{m}$; insets, $200 \mu \mathrm{m}$. 
Six3-Cre; $\boldsymbol{R} \boldsymbol{x}^{\text {flox } /-}$

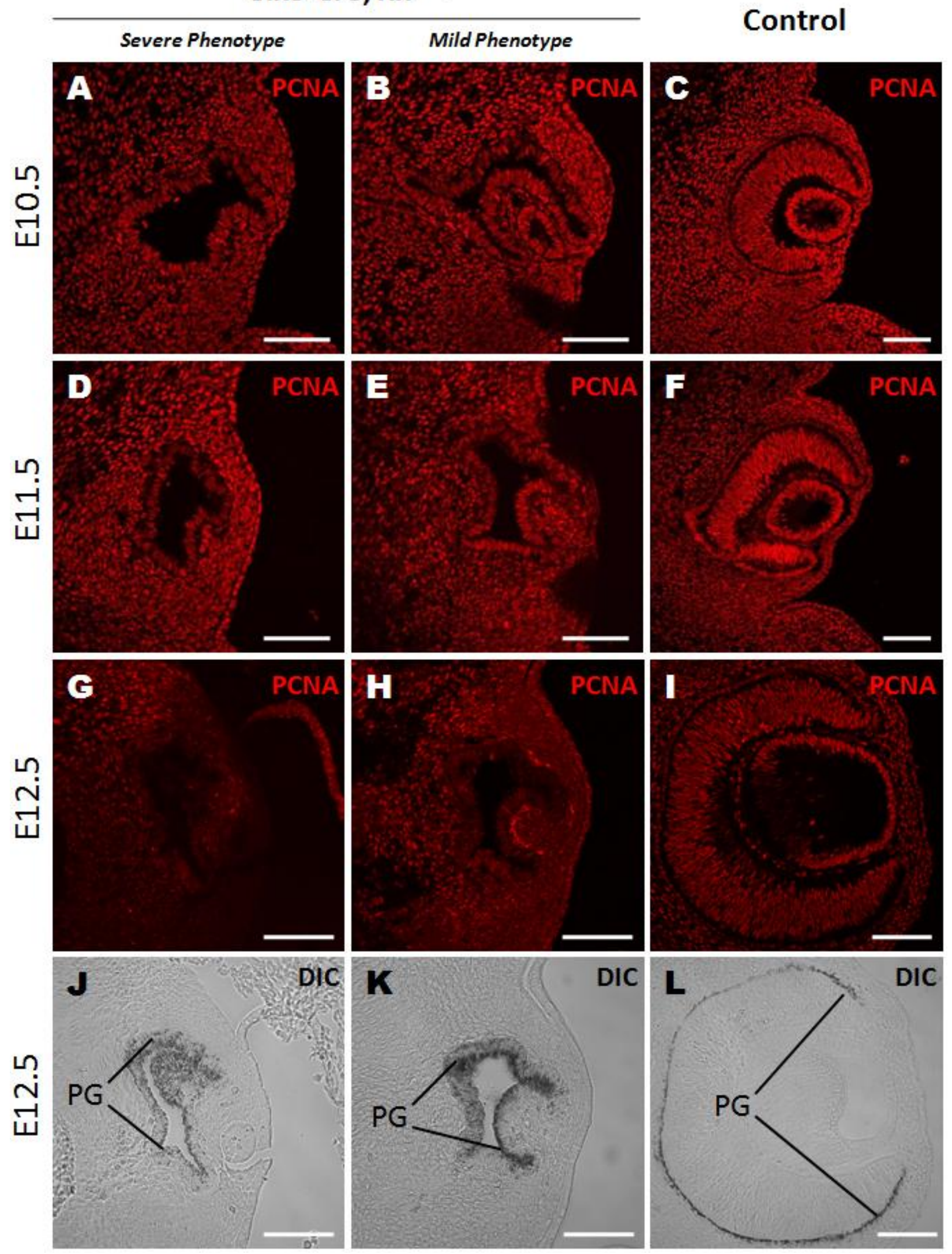


Figure 4. Decreased proliferation in the developing eye tissue of Six3-Cre; $R x$ conditional mutants versus control littermates. (A-I) Immunofluorescent labeling of proliferative cells with anti-proliferating cell nuclear antigen (PCNA) antibody in coronal cryosections through the orbit of Six3-Cre; $R x$ conditional mutants and littermate controls. Embryonic ages indicated at the left of each row. PCNA-positive cells are present throughout control presumptive eye tissue $(C, F, I)$. At E10.5 and E11.5, the persistent optic vesicle cells of Six3-Cre; $R x$ conditional mutants show reduced PCNApositive staining (A-B and D-E, respectively), while E12.5 persistent optic vesicle cells are virtually devoid of PCNA immunostaining $(\mathrm{G}-\mathrm{H})$. (J-L) DIC images of samples from G-I showing pigment deposition throughout the optic vesicle remnants of E12.5 Six3Cre; $R x$ conditional mutants (PG in J-K) and pigment deposition restricted to the RPE in the control (PG in L). PCNA, proliferating cell nuclear antigen; $P G$, pigment granules. Scale bars: $100 \mu \mathrm{m}$. 
$\square$ Cond. Mutant - Severe Phenotype $\square$ Control RPE

Cond. Mutant - Mild Phenotype $\square$ Control Neural Retina

A

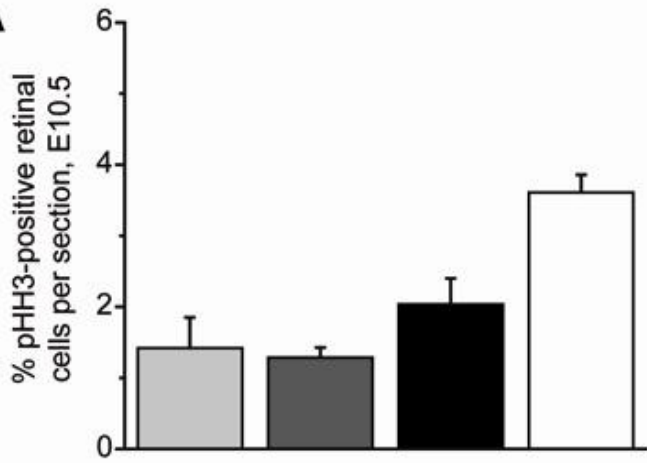

C

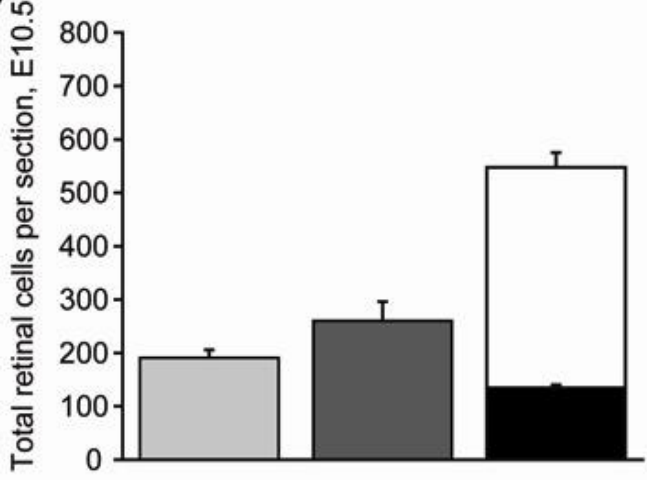

B

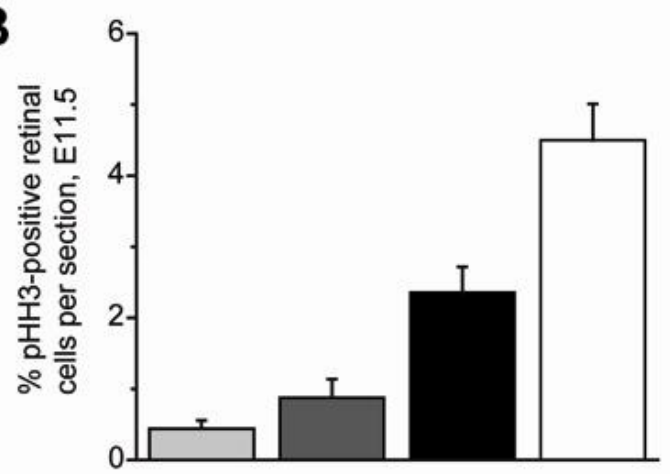

D

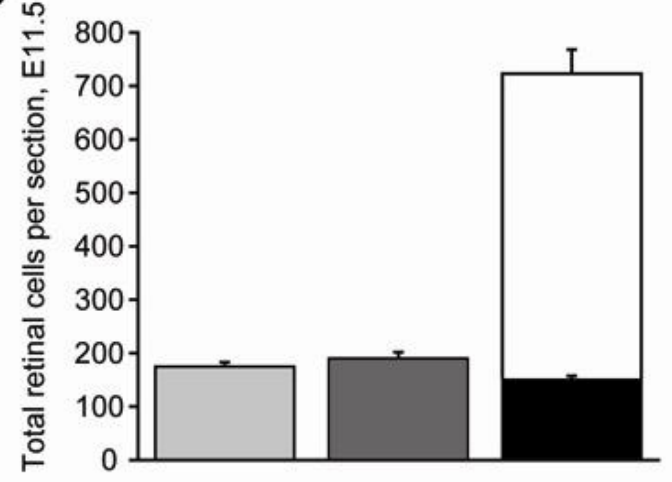

Six3-Cre; $R x^{\text {flox/- }}$

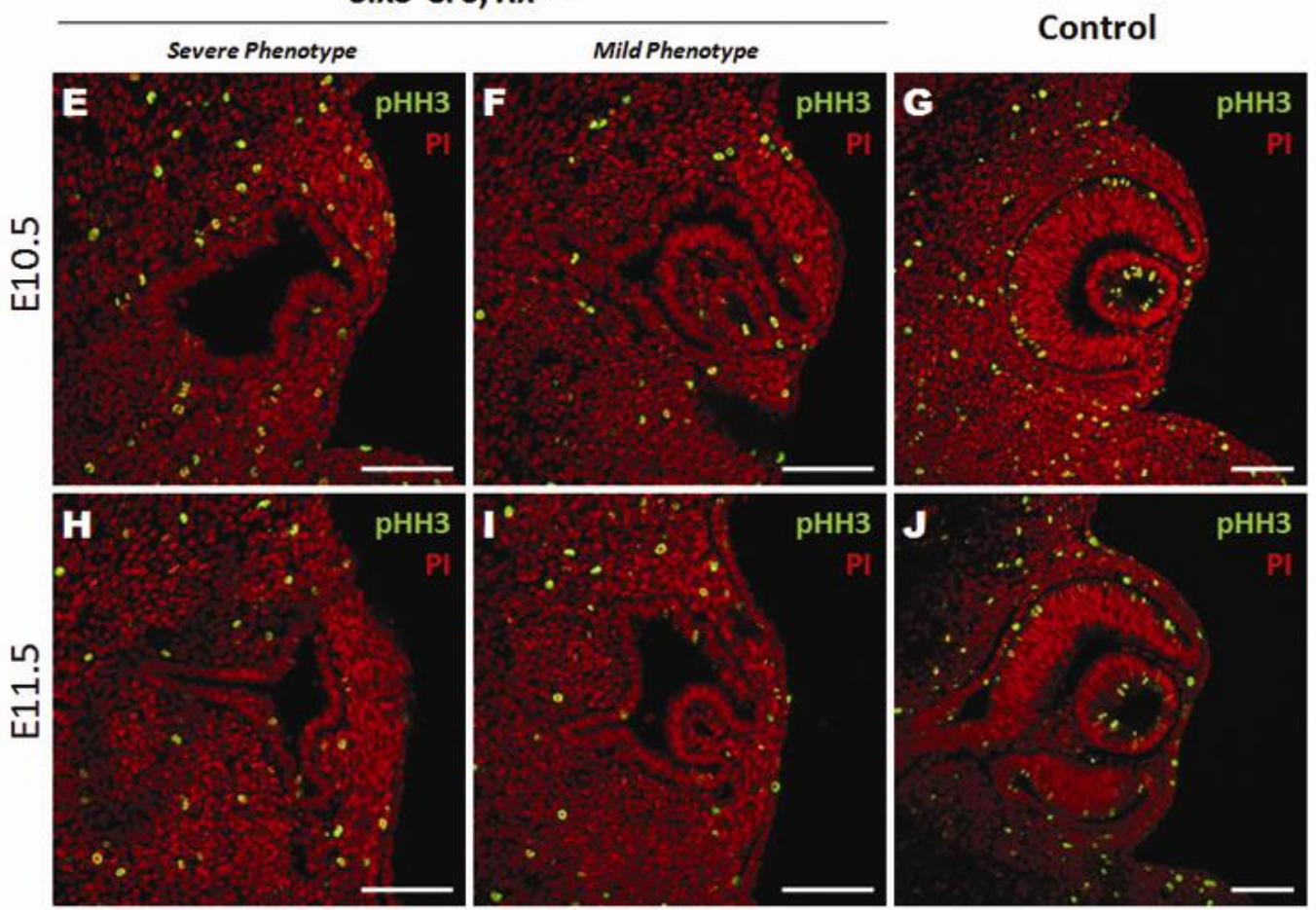


Figure 5. Decreased mitosis and total cell number in Six3-Cre; $R x$ conditional mutant optic vesicle remnants. (A-B) Histograms showing percentages of pHH3-positive cells within the persistent optic vesicles of E10.5 (A) and E11.5 (B) Six3-Cre; $R x$ conditional mutants and littermate control eye samples, per coronal section. (C-D) Histograms showing total retinal cell counts within the persistent optic vesicles of E10.5 (C) and E11.5 (D) Six3-Cre; $R x$ conditional mutants and littermate control eye samples, per section. Data represent mean \pm s.e.m. from at least 3 independent animals per group ( $n$ $\geq 3$ ); and at least 3 non-adjacent, central retinal $12 \mu \mathrm{m}$ sections were counted per animal. (E-J) Representative images of immunofluorescent staining with anti-pHH3 antibody and propidium iodide used to count cells. pHH3, phosphorylated histone $\mathrm{H} 3$; PI, propidium iodide. Scale bars, $100 \mu \mathrm{m}$. 


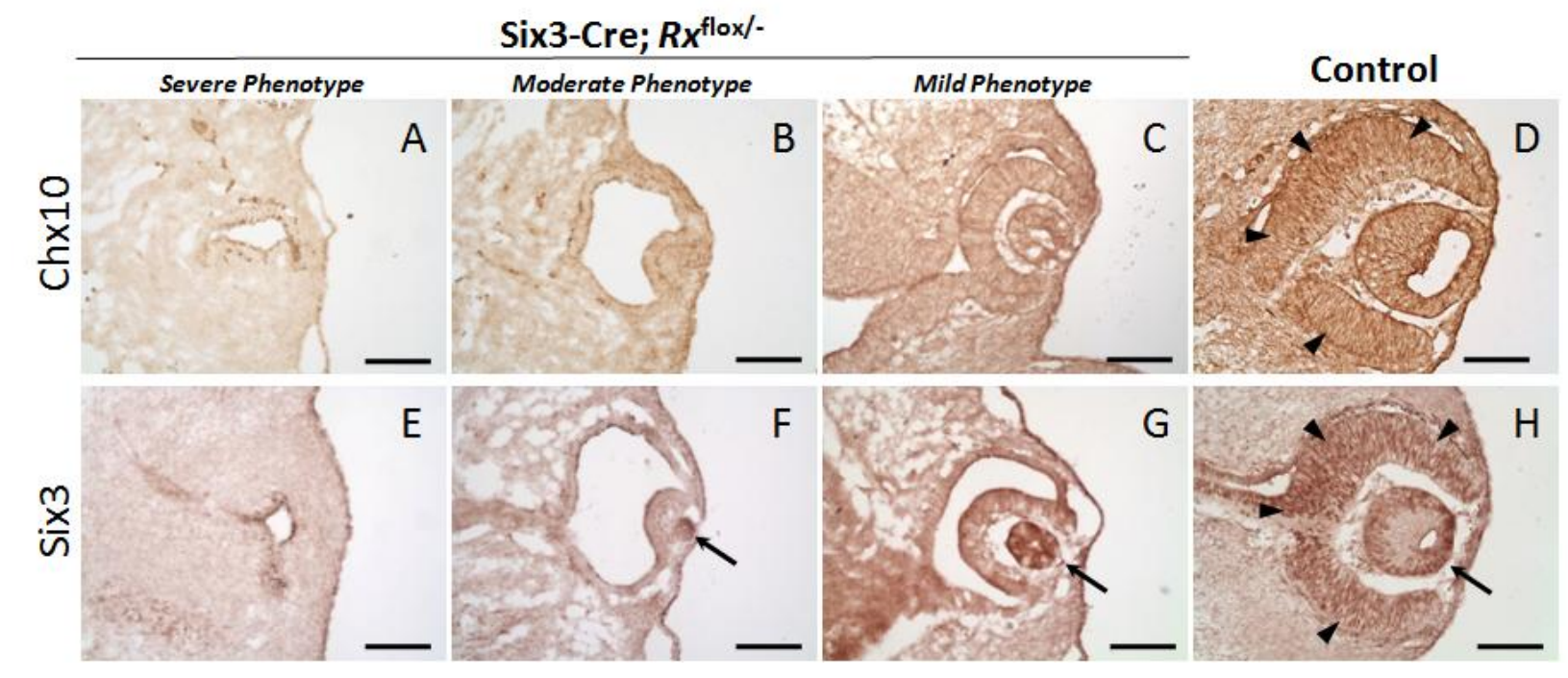

Figure 6. Six3-Cre-mediated deletion of $R x$ prevents the specification of neural retinal tissue. (A-H) Immunostaining for neural retinal cell markers, Chx10 and Six3, on E11.5 coronal sections through the orbit of Six3-Cre; $R x$ conditional mutants (A-C, and E-G) and control littermates $(\mathrm{D}$ and $\mathrm{H})$. Chx10 and Six3 immunostaining is absent from the persistent optic vesicles of conditional mutants, regardless of severity of phenotype (AC, E-G). Six3-positive staining is observed in the moderate and mild conditional mutants, within the surface ectoderm/presumptive lens vesicle adjacent to the presumptive optic cup (arrows in F and G). Arrowheads in D and $\mathrm{H}$ indicate Chx10- and Six3-positive staining, respectively, in the neural retina. Scale bars: $100 \mu \mathrm{m}$. 


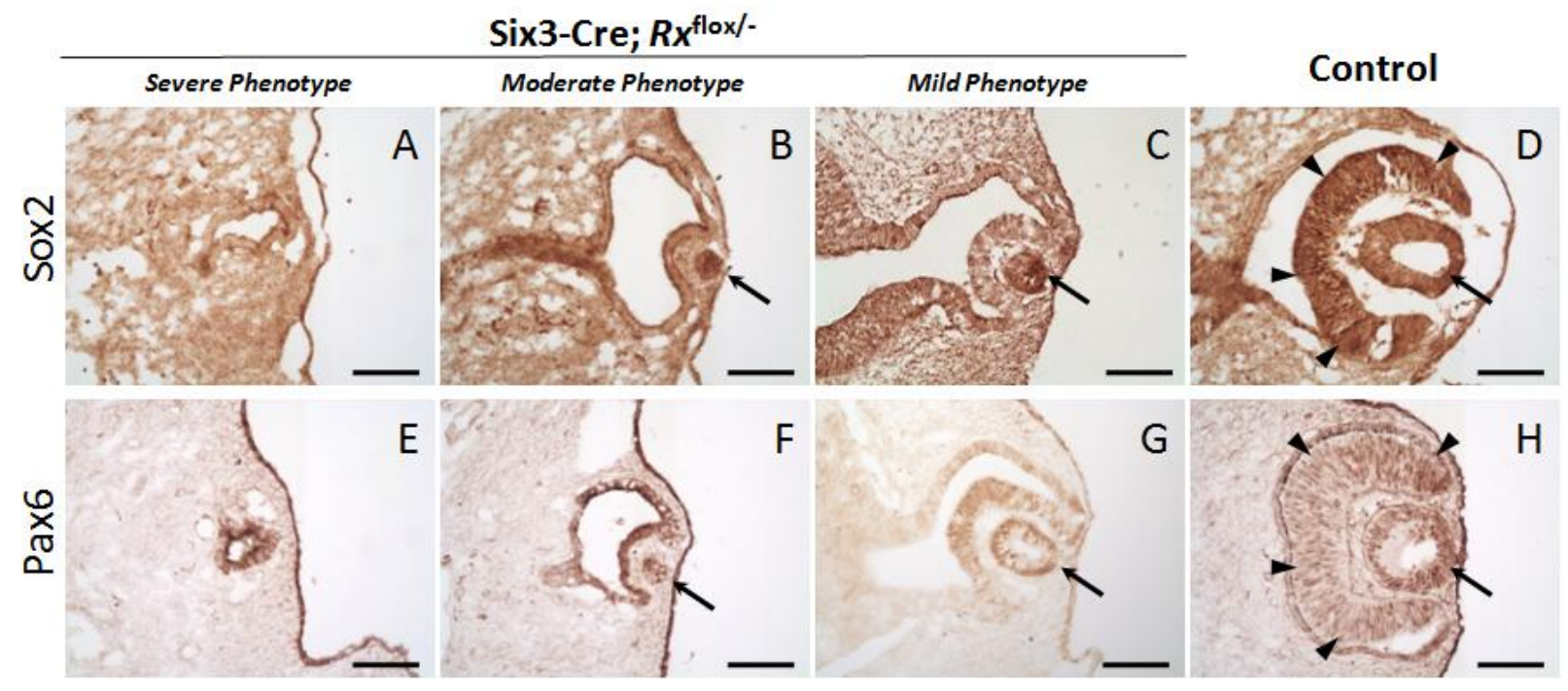

Figure 7. The moderate and mild phenotypes of the Six3-Cre; $R x$ conditional mutant allow for the initial specification of lens placode and lens vesicle, respectively. (A-H) Immunostaining of E11.5 coronal sections through the orbit of Six3-Cre; $R x$ conditional mutants (A-C, and $E-G)$ and control littermates $(D$ and $H$ ) using lens markers, Sox2 ( $A$ D) and Pax6 $(E-H)$. Whereas Sox2 immunolabeling is present in the neural retina (arrowhead) and lens vesicle (arrow) of the control embryonic eye (D), it is only present in the surface ectoderm of moderate and mild Six3-Cre; $R x$ conditional mutants, adjacent to the cupped distal optic vesicle (arrows in B and C). No Sox2 immunoreactivity is observed in the persistent optic vesicles of severely affected Six3Cre; $R x$ conditional mutants (A). At E11.5, Pax6 immunostaining is present in the RPE, NR (arrowheads) and lens (arrow) of control developing eyes $(H)$. The surface ectoderm adjacent to the optic cup, presumptive lens tissue, stains positively for Pax6 in the moderately and mildly affected Six3-Cre; $R x$ conditional mutants (arrows in F and G, respectively). Scale bars: $100 \mu \mathrm{m}$. 


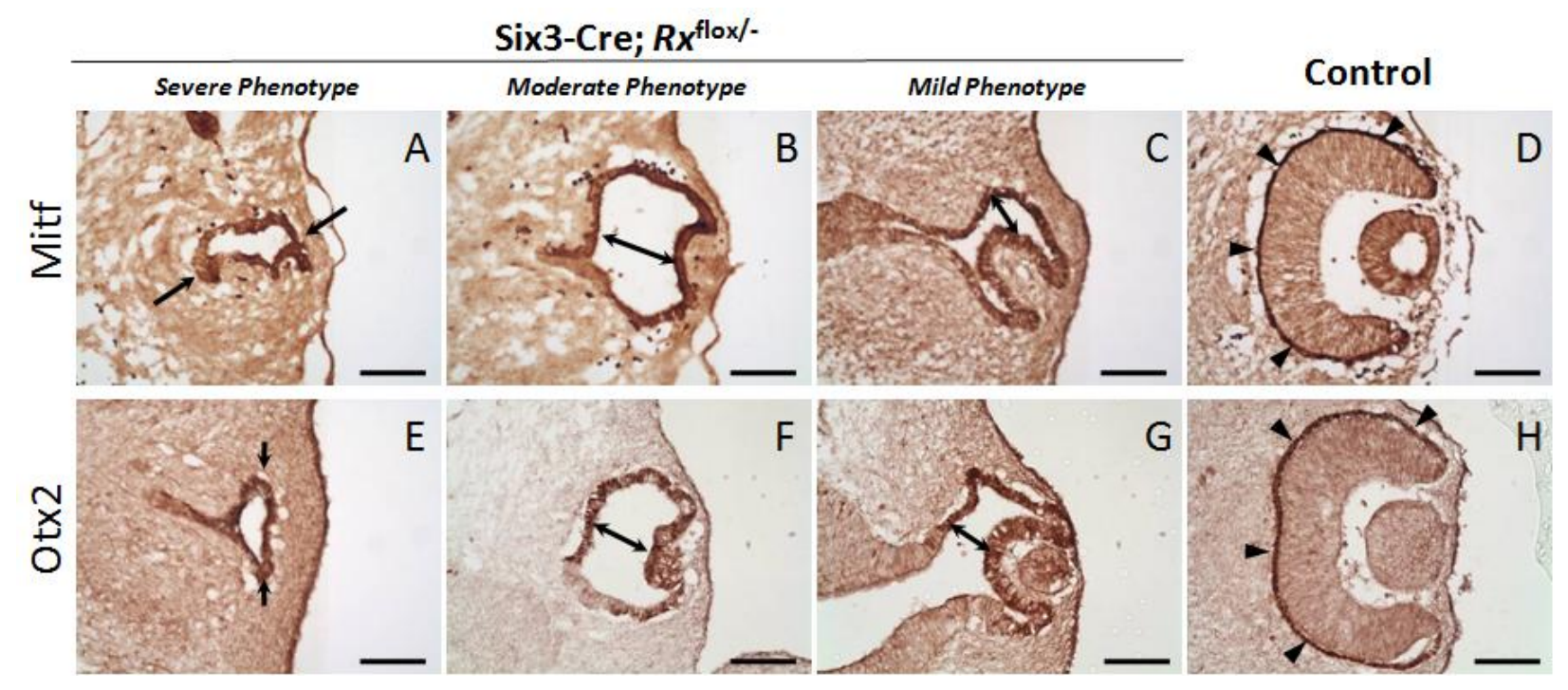

Figure 8. Six3-Cre-mediated inactivation of $R x$ results in a persistent optic vesicle/cup that adopts an RPE cell fate. (A-D) Immunostaining of E11.5 coronal sections through the orbit of Six3-Cre; $R x$ conditional mutants (A-C and E-G) and control littermates (D and $H)$ using RPE markers, Mitf $(A-D)$ and Otx2 $(E-H)$. Immunostaining with anti-Mitf and anti-Otx2 antibodies occurs in the RPE of E11.5 control eye sections (arrowheads in $\mathrm{H}$ and $\mathrm{L}$ ). Both Mitf- and Otx2-positive immunostaining are present throughout the persistent optic vesicles/cups of conditional mutants, including the distal-most/cupped region, regardless of phenotype severity (arrows in $\mathrm{A}-\mathrm{C}$ and $\mathrm{E}-\mathrm{H}$, respectively). Scale bars: $100 \mu \mathrm{m}$. 


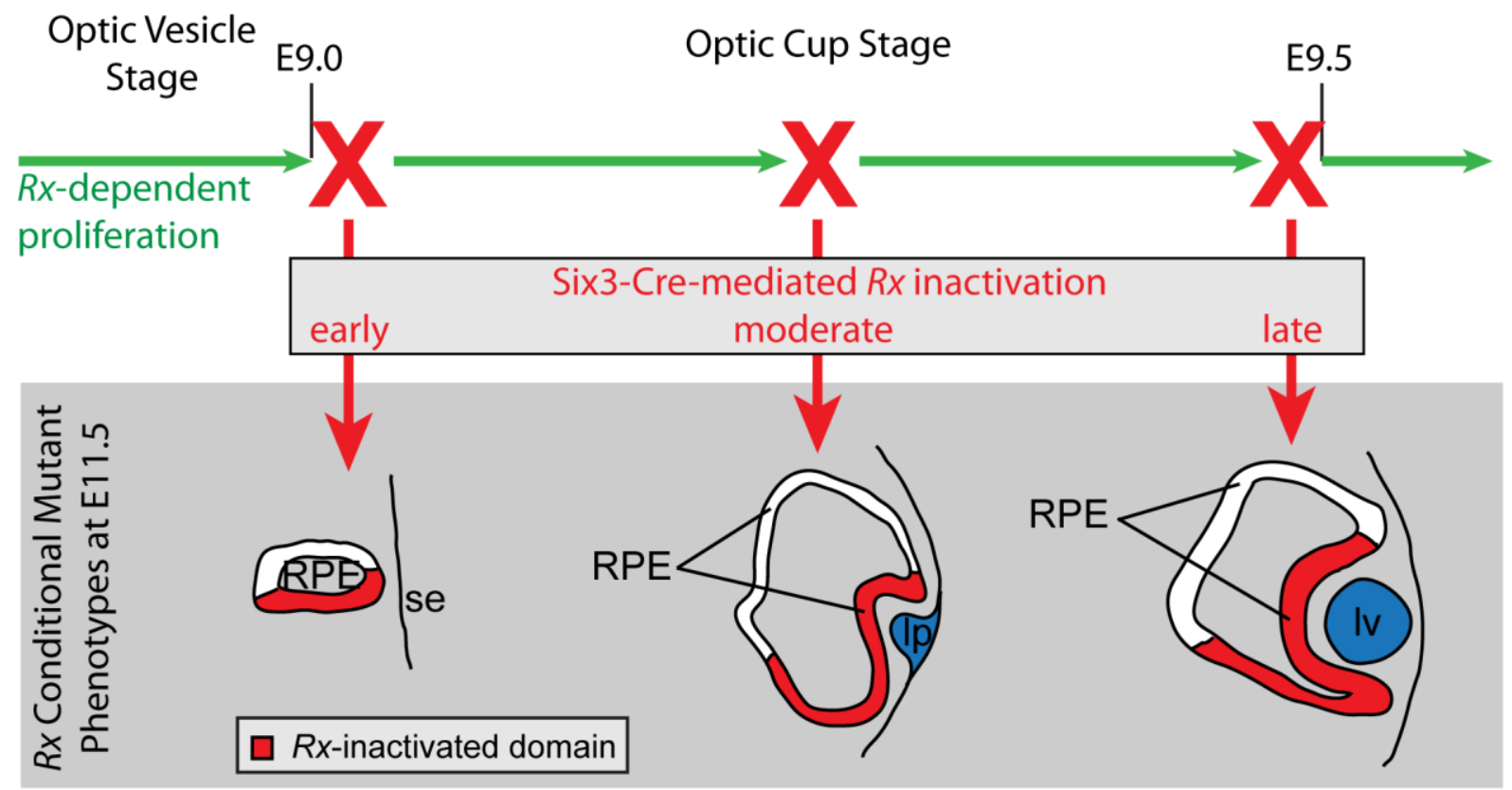

Figure 9. Model for the generation of variable developing eye phenotypes in Six3-Cre; $R x$ conditional mutant embryonic eyes. Phenotype severity is dependent on the timing of $R x$ inactivation by the Six3-Cre transgenic mouse model. Prolonged $R x$ activity (i.e. delayed Six3-Cre-mediated $\mathrm{Rx}$ inactivation) allows for progressive expansion of both the developing optic cup and lens, and a concomitant decrease in phenotype severity. The severe phenotype corresponds to early Six3-Cre-mediated Rx recombination and is characterized by no optic cup or lens placode formation. The moderate phenotype turns on Cre expression (inactivates $\mathrm{Rx}$ ) slightly later and is characterized by the formation of an underdeveloped optic cup and thickening of the lens placode. Finally, the mild phenotype corresponds to the most delayed onset of Six3-Cre expression and shows a more developed optic cup and a distinct lens vesicle. Despite these differences in developing eye morphology, neural retinal specification never occurs in any Six3-Cre; $R x$ conditional mutant optic vesicle/cup remnants, which assume an RPE identity throughout. 


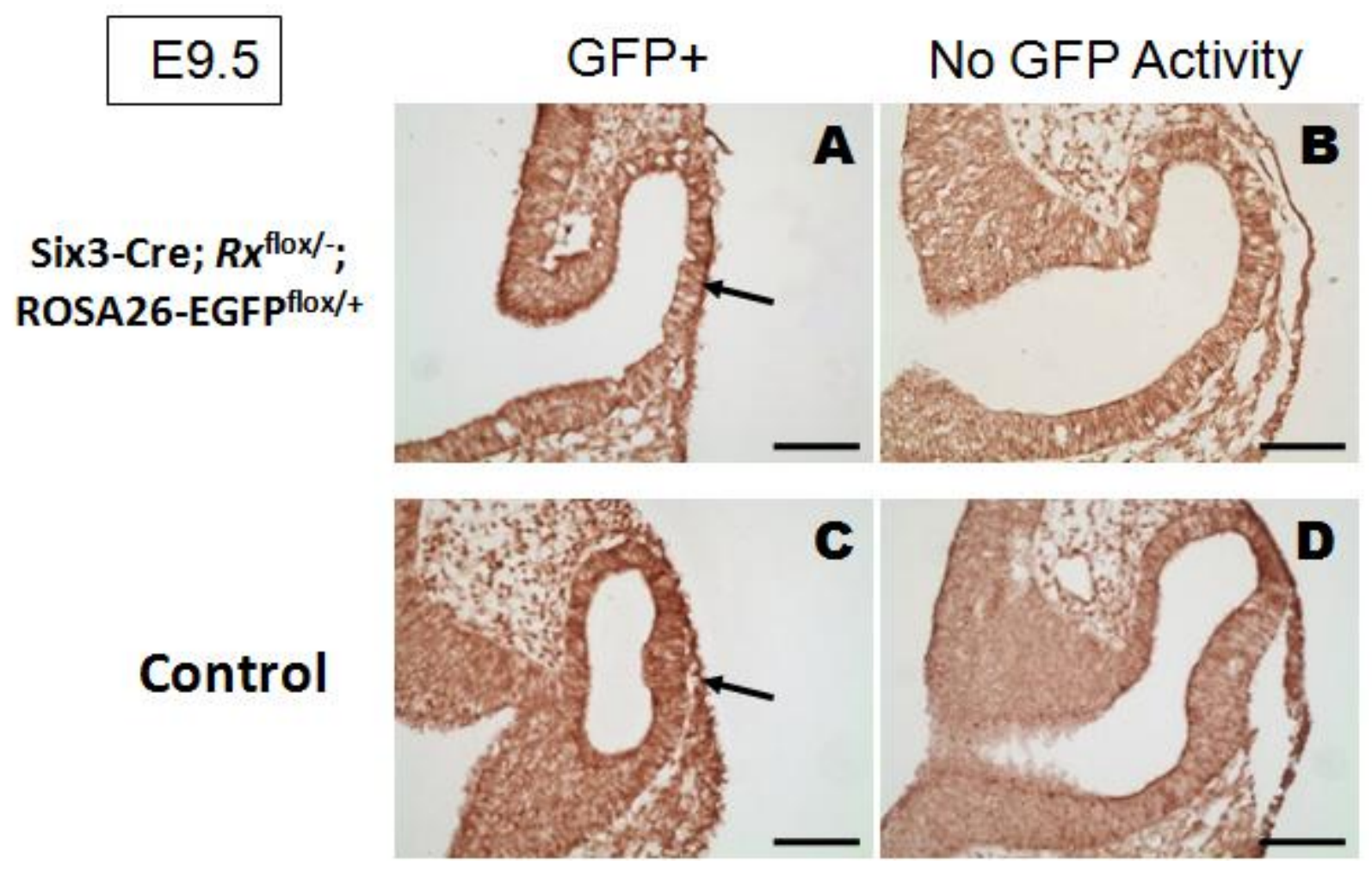

Supplemental Figure S1. Inconsistent Cre-mediated recombination/EGFP reporter activation in E9.5 optic vesicles by Six3-Cre transgenic model. (A-D) EGFP reporter activity assayed by immunostaining with an anti-GFP antibody on coronal sections through the orbit of E9.5 Six3-Cre; $R x$ conditional mutants and controls. Both conditional mutants (A-B) and controls (C-D) show inconsistent EGFP reporter activation in E9.5 optic vesicles. Arrows indicate GFP-positive immunostaining. Scale bars: $100 \mu \mathrm{m}$. 
Six3-Cre; $R x^{\text {flox } /-}$

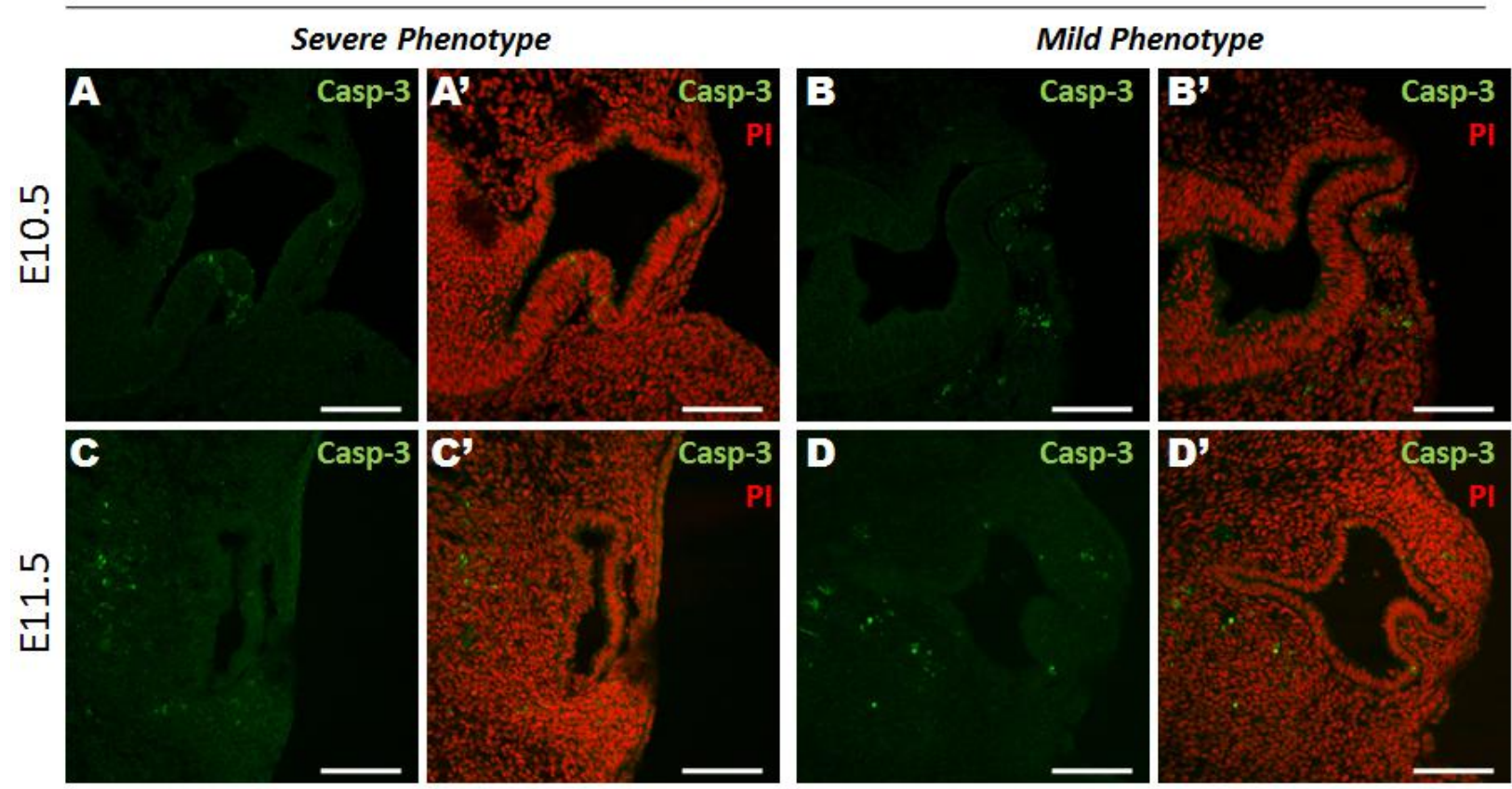

P8 Control Retina
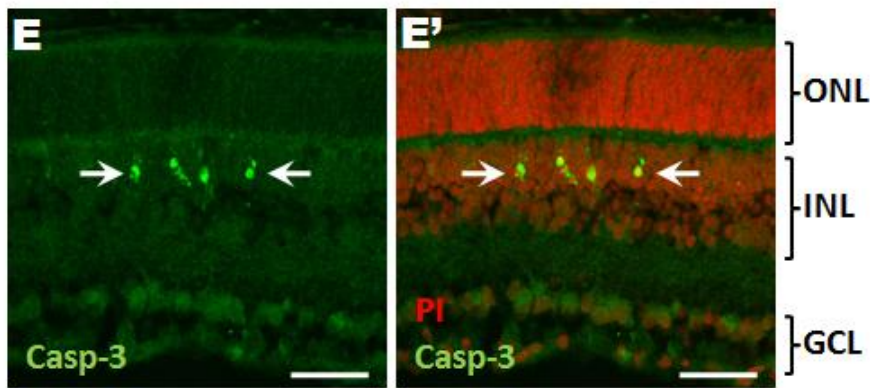

Supplemental Figure S2. No cell death in the developing eyes of E10.5 and E11.5 Six3-Cre; $R x$ conditional mutants. (A-E) Immunofluorescent labeling of coronal sections through the orbit of Six3-Cre; $R x$ conditional mutants (A-D) and P8 control retina (E) using apoptotic marker, activated Caspase-3 (Promega), and propidium iodide counterstain. Immunostaining with anti-activated Caspse-3 antibody is absent from the persistent optic vesicle/cup of both E10.5 and E11.5 conditional mutants, regardless of phenotype severity (A-D). Apoptotic cells are evident in the inner nuclear layer of P8 control retinas (arrows in E). Casp-3, Caspase-3; PI, propidium iodide; ONL, outer nuclear layer; INL, inner nuclear layer; GCL, ganglion cell layer. Scale bars: A-D, 100 $\mu \mathrm{m} ; \mathrm{E}, 50 \mu \mathrm{m}$. 


\section{Chapter III:}

Temporal requirement of $\boldsymbol{R} \boldsymbol{x}$ for FGF-mediated neural retinal specification 


\section{ABSTRACT}

Two different conditional deletion models of the retinal homeobox gene, $R x$, using the Foxg1Cre and the Six3-Cre mouse lines, demonstrate the importance of $R x$ in neural retinal specification and retinal progenitor cell proliferation (Voronina et al., unpublished manuscript; Zamora and Mathers, submitted manuscript). Conditional inactivation of $R x$ following optic vesicle evagination affects overall growth and morphology of the developing optic vesicle/cup, and prevents neural retinal formation, with resulting $R x$-mutant cells assuming an RPE cell fate. Ectopic expression of FGFs and downstream effectors of FGF-mediated signaling have the ability to transdifferentiate presumptive RPE cells to the neural retinal cell fate. Here we combine each of our $R x$ conditional deletion models with an RPE-to-neural retinal transdifferentiation model, the OVE1070 (FGF9) transgenic mouse (Zhao et al., 2001), to demonstrate that the timing of $R x$ inactivation dictates the developing eye's competence to undergo FGF-mediated neural retinal specification. Analysis of $R x^{\text {flox }}$; FGF9 compound mutant embryos with different onsets of $R x$ inactivation shows FGF9-mediated neural retinal specification can only be rescued in $R x$-mutant cells when $R x$ inactivation is delayed long enough to make cells competent to respond to FGF signaling. These data suggest $R x$ activity is required during the optic vesicle stage for the initial specification of neural retinal identity, but if $R x$ activity is maintained through the late optic cup stage, neural retinal fate is able to be specified in the absence of $R x$. We propose a model in which $R x$ activity is absolutely required to specify neural retinal cell identity until a temporal or developmental threshold is met during the optic cup stage, after which, neural retinal cell identity can be specified via FGF signaling, and independent of $R x$ activity.

\section{KEYWORDS}

$R x$, retina, transdifferentiation, specification, patterning, Foxg1-Cre, Six3-Cre, conditional mutant, OVE1070, FGF9, compound mutant, mouse

\section{INTRODUCTION}

The optic vesicle, an outgrowth of anterior neuroectoderm, is one of the earliest structures in eye development and is composed of progenitor cells that will either become neural retinal, retinal pigmented epithelial (RPE), or optic stalk cells. In normal development, the fate determination of neural retina, RPE and optic stalk is decided in a regionalized manner. The most distal portion of the optic vesicle makes contact with the surface ectoderm and becomes neural retina. The dorsal optic vesicle gives rise to RPE, and the most proximal portion of the 
optic vesicle narrows and becomes the optic stalk. This patterning can be altered through the introduction of signaling molecules or the manipulation of various developmentally regulated transcription factors. This has been extensively demonstrated in the distal and dorsal optic vesicle, where fate determination of neural retina and RPE can be interchanged: presumptive RPE cells can transdifferentiate into neural retinal cells, and vice versa, demonstrating the bipotentiality of optic vesicle cells (reviewed in Tsonis and Del Rio-Tsonis, 2004; Zhao et al., 1997).

FGF signaling in early retinal development has been identified as a key regulator of neural retinal fate determination. FGFs 1 and 2, emanating from the surface ectoderm, direct the establishment of the optic cup and neural retinal domains (Hyer et al., 1998; Nguyen and Arnheiter, 2000; Pittack et al., 1997). Further, ectopic expression of FGF family members or downstream effectors of the FGF signaling pathway can cause transdifferentiation of presumptive RPE cells into the neural retinal cell fate (Galy et al., 2002; Guillemot and Cepko, 1992; Park and Hollenberg, 1989; Pittack et al., 1991; Vogel-Hopker et al., 2000; Zhao et al., 2001; Zhao and Overbeek, 1999). In particular, transgenic mice that ectopically express FGF9 in the presumptive RPE exhibit transdifferentiation of that tissue into a duplicate, mirror-image neural retina (Zhao et al., 2001; Zhao and Overbeek, 1999). However, in mouse embryos lacking FGF9, the neural retinal and RPE tissues still form, but the boundary between the two is shifted such that RPE cells are found in the distal, peripheral edges of the neural retina (Zhao et al., 2001). Therefore, while FGF9 is not absolutely required for neural retinal specification, its ectopic activity is sufficient to direct the specification of neural retina. Indeed, FGF9-mediated conversion of presumptive RPE to NR activates the expression of $R x$ (Zhao et al., 2001), a 
homeobox transcription factor expressed in the early neural retina but not the RPE (Furukawa et al., 1997; Mathers et al., 1997).

Germline deletion of $R x$ prevents formation of the optic vesicle from the anterior neuroectoderm during embryogenesis (Mathers et al., 1997). However, conditional inactivation of $R x$ during the optic vesicle and optic cup stages, through Foxg1-Cre- and Six3-Cre-mediated recombination of the $R x^{\text {flox }}$ allele (Voronina et al., 2005), respectively, shows that $R x$ function is required for the proliferation of retinal progenitors and for the establishment of neural retinal cell identity (Voronina et al., unpublished manuscript; Zamora and Mathers, submitted manuscript). Both $R x$ conditional inactivation models result in a persistent optic vesicle/cup that adopts an RPE fate. Interestingly, in Foxg1-Cre; Rx conditional mutant optic vesicles, expression of FGF8 and FGF15 are downregulated (Voronina et al., unpublished manuscript), suggesting $R x$ may lie upstream of FGF signaling in the genetic pathway required for neural retinal specification.

The OVE1070 transgenic mouse strain is an ectopic FGF9-expressing model that was originally designed to drive FGF9 expression in the developing lens, using a lens-specific, $\alpha$ Acrystallin promoter (Lovicu and Overbeek, 1998; Zhao et al., 2001). However, probably due to genome-integration-site effects, the FGF9 transgene is also ectopically and transiently expressed in the presumptive dorsal RPE from E10.5-11.5, respecifying cells in this region toward the neural retinal cell fate (Zhao et al., 2001). We obtained this FGF9-expressing transgenic mouse to test whether ectopic FGF expression in our two $R x$ conditional deletion models could rescue neural retinal cell formation in the absence of $R x$ activity.

Here, through the inactivation of $R x$ at two different stages of early embryonic eye development, we describe a time-dependent requirement for $R x$ activity in the establishment of neural retinal cell identity. We show that ectopic expression of FGF9 in the Foxg1-Cre; $R x$ 
conditional mutant (in which $R x$ is inactivated during the optic vesicle stage) cannot rescue neural retina formation, while delayed $R x$ inactivation, using the Six-Cre; $R x$ conditional mutant

model, can allow for FGF9-mediated neural retinal specification, depending on how long $R x^{\text {flox }}$ is able to promote its proliferative functions before its Six3-Cre-mediated inactivation. We propose a model in which delayed $R x$ inactivation during the optic cup stage allows cells to respond to ectopic FGF expression and rescue neural retinal formation independent of $R x$ activity. We envision a developmental or temporal threshold, during the optic cup stage, after which $R x$ activity may not be required for FGF-mediated neural retinal specification.

\section{Materials and Methods:}

\section{Generation of Foxg1-Cre and Six3-Cre; Rx conditional + OVE1070 compound}

mutants. Foxg1-Cre transgenic mice (gift from Dr. Mark Lewandowski, National Cancer Institute, Frederick, MD) and Six3-Cre transgenic mice (strain \#69, gift from Dr. Guillermo Oliver, St Jude's Research Hospital, Memphis, TN) were crossed with mice from our $R x$-null strain to create Foxg1-Cre; $R x^{+/-}$and Six3-Cre; $R x^{+/-}$animals, as described previously (Voronina et al., unpublished; Zamora and Mathers, submitted manuscript). The Foxg1-Cre, Six3-Cre and $R x$-null strains are as described previously (Furuta et al., 2000; Hebert and McConnell, 2000; Mathers et al., 1997).

Foxg1-Cre; $R x^{+/-}$and Six3-Cre; $R x^{+/-}$animals were then crossed with OVE1070 transgenic mice (gift from Dr. Paul Overbeek, Baylor College of Medicine, Houston, TX) to generate both Foxg1-Cre; $R x^{+-}$; OVE1070, and Six3-Cre; $R x^{+/}$; OVE1070 males. Finally, Foxg1-Cre; $R x^{+/-}$; OVE1070, and Six3-Cre; $R x^{+/-}$; OVE1070 males were crossed to $R x^{\text {flox/flox }}$; ROSA26-EGFP ${ }^{\text {floxfflox }}$ females (Mao et al., 2001; Voronina et al., 2005) to generate our embryos 
of interest, Foxg1-Cre; $R x^{\text {flox/- }}$ OVE1070; Rosa26-EGFP ${ }^{\text {flox/+ }}$ (Foxg1-Cre; $R x^{\text {flox }}$; FGF9 compound mutants), and Six3-Cre; $R x^{\text {flox/- }}$ OVE1070; Rosa26-EGFP ${ }^{\text {flox/+ }}$ (Six3-Cre; $R x^{\text {flox }}$; FGF9 compound mutants). These test crosses produce each $R x^{\text {flox }}$; FGF9 compound mutant genotype in $12.5 \%$ (1/8) of the progeny. The control littermates analyzed were Foxg1-Cre; $R x^{\text {flox/+}} ;$ Rosa26-EGFP ${ }^{\text {flox } /+}$ and Six3-Cre; $R x^{\text {flox/+}} ;$ Rosa26-EGFP ${ }^{\text {flox/+ }}$ mice, which are phenotypically wild-type and allow for EGFP reporter analysis of the corresponding Creexpressing strain. The OVE1070 controls were the genotype Foxg1-Cre; $R x^{\text {flox/+}}$; OVE1070; Rosa26-EGFP ${ }^{\text {flox/+}}$, or Six3-Cre; $R x^{\text {flox/+}}$; OVE1070; Rosa26-EGFP ${ }^{\text {flox/+ }}$. The $R x$ conditional mutant ( $R x$ CKO, [Conditional KnockOut]) controls were either Foxg1-Cre; $R x^{\text {flox/- }}$; Rosa26$\mathrm{EGFP}^{\mathrm{flox} /+}$, or Six 3-Cre; $R x^{\mathrm{flox} /-} ;$ Rosa26-EGFP ${ }^{\mathrm{flox} /+}$, and are as described previously (Voronina et al., unpublished; Zamora and Mathers, submitted manuscript).

PCR genotyping of compound mutants and controls. Tail biopsies were collected and digested in a PCR-competent lysis solution $(50 \mathrm{mM} \mathrm{KCl}, 10 \mathrm{mM}$ Tris $\mathrm{HCl} \mathrm{pH}$ 8.3, $2 \mathrm{mM} \mathrm{MgCl}$, $0.45 \%$ IGEPAL CA- $630,0.45 \%$ Tween-20) plus proteinase $\mathrm{K}(0.6 \mathrm{mg} / \mathrm{mL})$ overnight at $55^{\circ} \mathrm{C}$, to extract genomic DNA from embryos and pups. PCR reactions were done using the Cre, $R x$ and SV40 pA (OVE1070) oligonucleotide primers and conditions previously described (Holzenberger et al., 2000; Lovicu and Overbeek, 1998; Voronina et al., 2005).

Morphologic and Immunohistologic Analyses. Embryonic heads were fixed in 4\% paraformaldehyde overnight at $4{ }^{\circ} \mathrm{C}$, cryoprotected in $30 \%$ sucrose in PBS, and cryosectioned coronally at $12 \mu \mathrm{m}$. Developing ocular morphology was analyzed by Nuclear Fast Red staining and DIC microscopy. Immunohistochemistry was performed on cryosections. First, epitope unmasking was done by incubating sections in $0.1 \mathrm{M}$ Tris- $\mathrm{HCl}, \mathrm{pH} 9.5$ at $95^{\circ} \mathrm{C}$, for $20 \mathrm{~min}$. The sections were blocked for 90 min in normal serum, incubated overnight with primary antibodies 
at $4^{\circ} \mathrm{C}$, washed with PBS, incubated for $2 \mathrm{~h}$ at room temperature with either biotinylated or Alexa-conjugated secondary antibodies, and washed in PBS. Immunostaining was visualized by either using an $\mathrm{ABC}$ Elite kit (Vector Labs) and diaminobenzidine staining for biotinylated secondary antibody reactions, or Zeiss LSM 510 Meta confocal microscopy for Alexaconjugated secondary antibody reactions. Primary antibodies used were GFP (1:1000, Molecular Probes), di-phosphorylated ERK1/2 (p42/44, "pERK") (1:300, Cell Signaling); Chx10 (1:50200, ExAlpha), Mitf (1:1000, gift from Arnheiter lab), Otx2 (1:1000, Chemicon), Sox2 (1:500, Chemicon), Pax6 (1:50, Developmental Studies Hybridoma Bank, Univ. of Iowa), Six3 (1:500, gift from Oliver lab), and Cyclin D1 (1:200, Santa Cruz). ). All immunohistochemical and immunofluorescent reactions were done on at least three different biological replicates for each genotype and age tested $(n \geq 3)$.

In situ hybridization. The expression pattern of transgenic FGF-9 transcripts in the eye was examined by in situ hybridization using a DIG-labeled riboprobe containing SV40 pA sequences. To visualize in situ hybridization, tissue sections were incubated with alkaline phosphatase-conjugated, anti-DIG antibody (Roche), overnight at $4^{\circ} \mathrm{C}$. Unbound antibody was rinsed away and slides were developed by adding BM Purple, a chromogenic substrate for alkaline phosphatase. After development, sections were refixed (4\% Paraformaldehyde, $0.1 \%$ Glutaraldehyde), dehydrated and mounted with a coverslip for examination. Bright-field images were collected using a CCD camera (Optronics Engineering) mounted on an Olympus microscope. 


\section{$\underline{\text { RESULTS }}$}

\section{Addition of ectopic FGF9 fails to rescue neural retinal specification in Foxg1-Cre; $R x$ conditional mutants.}

Previous work in our lab has demonstrated that $R x$ is required during the optic vesicle stage for neural retinal cell fate determination (Voronina et al., unpublished manuscript; Zamora and Mathers, submitted manuscript). Using the Foxg1-Cre and Six3-Cre animal models (Furuta et al., 2000; Hebert and McConnell, 2000) in conjunction with our $R x^{\text {flox }}$ animal model (Voronina et al., 2005), we have shown that conditional inactivation of $R x$ during the optic vesicle and optic cup stage decreases retinal progenitor cell proliferation, halts the progression of eye development, and results in a pigmented optic vesicle or cup remnant composed entirely of RPE-fated cells (Voronina et al., unpublished manuscript; Zamora and Mathers, submitted manuscript). That work, as well as other experiments, led to the hypothesis that $R x$ is required for the specification of neural retina. Other studies have established FGFs and their signaling pathways as important mediators of neural retinal specification (Hyer et al., 1998; Nguyen and Arnheiter, 2000; Pittack et al., 1997). Thus, in order to further understand the relationship between $R x$ activity and FGF signaling in the genetic pathway required to specify neural retina, we sought to genetically combine an FGF-mediated, RPE-to-neural retinal transdifferentiation model, with our two $R x$ conditional mutant models: the Foxg1-Cre; $R x$ conditional mutant and the Six3-Cre; $R x$ conditional mutant. To create these compound mutant models, we obtained the OVE1070 (FGF9) animal strain, in which FGF9 is transiently and ectopically expressed in the developing dorsal RPE and the lens, and results in transdifferentiation of the dorsal RPE into neural retina (Zhao et al., 2001; Fig. 1B and F).

To first test whether neural retinal specification via FGF-mediated transdifferentiation

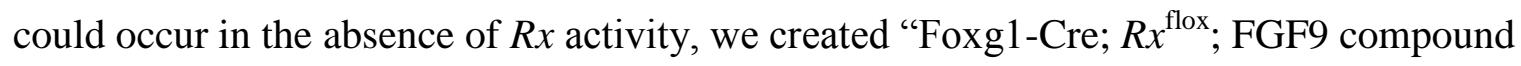




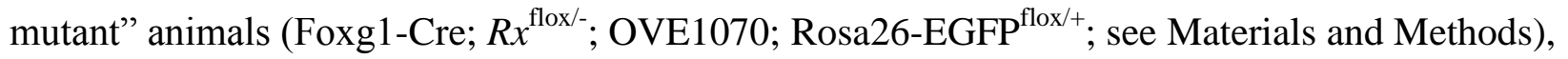
which also carry a Rosa26-EGFP reporter allele to lineage-trace cells that have undergone Cremediated recombination. From test crosses to produce these compound mutants, we collected embryos and controls spanning the early stages of retinal development (E9.5-E12.5) and analyzed their morphology grossly and histologically. Addition of the FGF9 transgene to the Foxg1-Cre; $R x$ conditional mutant model fails to change its phenotype from that of a pigmented optic vesicle remnant as analyzed by whole-mount and Nuclear Fast Red-stained sections (compare Fig. 1D and H to Fig. 1C and G).

The lack of a phenotypic change with the introduction the FGF9 transgene, in the Foxg1Cre; $R x^{\text {flox }}$ FGF9 compound mutants, suggests $R x$ activity is required for FGF-mediated neural retinal specification, but could also have resulted from an inability of the FGF transgene to be expressed in the Foxg1-Cre; $R x$ conditional mutant background. To verify that both elements of the Foxg1-Cre; $R x^{\text {flox }}$; FGF9 compound mutant-Cre-mediated $R x^{\text {flox }}$ recombination and the FGF9 transgene- are present and working properly in compound mutant embryos, we performed Rosa26-EGFP reporter assays (Fig. 2) and in situ hybridization reactions using an FGF9 transgene-specific, antisense RNA probe (SV40; Fig. 3A-D), on compound mutants and controls. Rosa26-EGFP reporter activity is present in the developing optic vesicles of Foxg1-Cre-positive E9.5 samples (Fig. 2A-D). As development progresses to E11.5, Rosa26-EGFP reporter activity is maintained in the resulting optic vesicle remnant tissue of both Foxg1-Cre; $R x^{\text {flox }}$ conditional mutants and Foxg1-Cre; $R x^{\text {flox; }}$ FGF9 compound mutants (arrows in Fig. 2G-H), suggesting Foxg1-Cre-mediated $R x^{\text {flox }}$ recombination/inactivation has occurred in the optic vesicle remnant cells in each animal model. As expected, in situ hybridizations on E11.5 OVE1070 (FGF9) samples exhibited transgenic expression of FGF9/SV40 transcript in the dorsal RPE- 
transdifferentiated neural retina and in the posterior lens vesicle (arrows in Fig 3B; Zhao et al., 2001). Foxg1-Cre; $R x^{\text {flox }}$; FGF9 compound mutants exhibit FGF9/SV40 transgenic expression in the dorsal portion of their resulting optic vesicle remnants (arrow in Fig. 3D), indicating ectopic expression of the FGF9 transgene occurs within the $R x$ conditional mutant background. Expression of transgenic FGF9/SV40 transcript was absent from E11.5 controls and Foxg1-Cre; $R x$ conditional mutants, as expected (Fig. 3A and C)

FGF-mediated transdifferentiation of presumptive RPE cells to neural retinal cells has been shown to work through the Ras-Raf-MEK-ERK pathway (Galy et al., 2002; Spence et al., 2007; Zhao et al., 2001), allowing for FGF signaling to be monitored by downstream ERK activation (Corson et al., 2003). To further confirm the FGF9 transgene is present and active, and to test whether ectopic FGF9 in the Foxg1-Cre; $R x$ conditional mutant background is activating the Ras-Raf-MEK-ERK pathway, we mapped activated ERK signaling domains within the developing orbits of Foxg1-Cre; $R x^{\text {flox }}$ FGF9 compound mutants and controls via immunofluorescent staining with an antibody specific to the active, di-phosphorylated forms of ERK1 and ERK2 (pERK; Fig. 3E-H; Corson et al., 2003). Immunofluorescent staining for pERK is absent from the RPE, but present in the neural retina and lens vesicle of E11.5 control eyes, (Fig. 3E), indicating ERK is activated in the neural retina and lens vesicle during normal eye development (Corson et al., 2003). In addition to staining in the neural retina, pERK staining is also present in the dorsal RPE region that has transdifferentiated into neural retina for FGF9 control eyes, while the untransdifferentiated RPE remains unstained for pERK (Fig. 3F). Increased pERK staining is present in the lens vesicle of FGF9 eyes, as compared to wild-type control eyes, due to the ectopic FGF9 expression in the lens vesicle (Fig. 3F). No pERK staining is observed in the optic vesicle remnant of Foxg1-Cre; $R x$ conditional mutants (Fig. 3G), whereas 
Foxg1-Cre; $R x^{\text {flox}}$; FGF9 compound mutants show pERK staining within their optic vesicle remnants (arrows in Fig. 3H), confirming the activation of ERK signaling in the absence of $R x$ activity, following the genetic addition of ectopic FGF9 expression.

To assess whether the ectopic addition of FGF9 is able to change the RPE expression profile and transdifferentiate RPE cells populating the optic vesicle remnants in Foxg1-Cre; $R x$ conditional mutants, we performed immunohistologic analyses with antibodies against markers

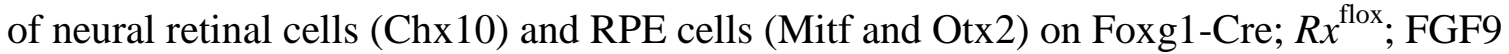
compound mutants and controls. Neither Foxg1-Cre; Rx conditional mutant, nor Foxg1-Cre;

$R x^{\text {flox}}$; FGF9 compound mutant optic vesicle remnants showed staining for Chx10 (Fig. 4C and D). Instead, the optic vesicle remnants of both Foxg1-Cre; $R x$ conditional and compound mutants stained throughout for RPE markers, Mitf and Otx2 (Fig. 4G, H, K, and L). Taken together, these data indicate ectopic FGF9 signaling is not sufficient to respecify or transdifferentiate RPE cells to a neural retinal cell fate in the Foxg1-Cre; $R x$ conditional mutant background, and that $R x$ activity during the optic vesicle stage is required for FGF9-mediated neural retinal specification.

\section{Addition of ectopic FGF9 changes the developing morphology of Six3-Cre; $R x$ conditional mutant eyes.}

We have previously described another $R x$ conditional mutant animal model, the Six3-Cre; $R x$ conditional mutant, in which $R x$ is inactivated approximately a day later (E9.0-9.5) than the Foxg1-Cre; $R x$ conditional mutant, in the developing optic cup (Zamora and Mathers, submitted manuscript). Similar to the Foxg1-Cre; $R x$ conditional mutant model, inactivation of $R x$ using the Six3-Cre transgenic model causes cells of the developing optic vesicle to assume an RPE fate; however, it does allow for some progression of early ocular morphology_optic cup formation 
and early lens development. Since the Six3-Cre transgenic model expresses Cre recombinase about half a day to a day later than the Foxg1-Cre animal model, we wanted to test whether this prolonged $R x$ activity makes the developing optic tissue more responsive to ectopic FGF9mediated neural retinal respecification. Using the same genetic strategy employed to create the Foxg1-Cre; $R x^{\text {flox }}$ FGF9 compound mutant model, we created the Six3-Cre; $R x^{\text {flox }}$ FGF9 compound mutant model (Six3-Cre; $R x^{\text {flox/- }}$; OVE1070; Rosa26-EGFP ${ }^{\text {flox/+_}}$; see Materials and Methods) to assess the temporal importance of $R x$ expression and activity during early retinal determination.

Whole-mount inspection of E12.5 Six3-Cre; $R x^{\text {flox }}$; FGF9 compound mutant eyes reveals a loss of pigment in the dorsal hemisphere and an overall size that is intermediate between that of WT controls and Six3-Cre; $R x$ conditional mutants (Fig. 5D). More detailed morphologic inspection of E12.5, Six3-Cre; $R x$ conditional mutant sections exhibit an optic vesicle remnant that has taken an RPE fate and is pigmented throughout (Fig. 5G; Zamora and Mathers, submitted manuscript), while age-matched Six3-Cre; $R x^{\text {flox }}$; FGF9 compound mutant sections show tissue thickening and loss of pigment deposition in two distinct, distal eye areas: the distaldorsal region (arrow in Fig. 5H) and the distal-ventral region cupping the developing lens (arrowhead in Fig. 5H). Interestingly, we observed the transdifferentiation phenotype in the distal-dorsal region in $~ 88 \%$ of the compound mutant eyes analyzed (21/24). In the distal-ventral region, tissue thickening and loss of pigmentation occurred in $\sim 67 \%$ of the compound mutant eyes analyzed (16/24), and seemed to correlate with the formation and growth of lens vesicle. Six3-Cre; $R x^{\text {flox}}$; FGF9 compound mutants that lack a distinct lens vesicle, or form a comparatively undersized lens vesicle, do not show tissue thickening or loss of pigmentation in the distal-ventral region (black arrow in Fig. 5I), but do maintain both morphological changes in 
the distal-dorsal region (blue arrow in Fig. 5I). Notably, we did observe a small percentage of Six3-Cre; $R x^{\text {flox }}$ FGF9 compound mutant eyes (3/24) whose morphology was similar to those of severely affected Six3-Cre; $R x$ conditional mutants and failed to show any morphological changes in the distal region (Fig. 5L; Zamora and Mathers, submitted manuscript). Because these rare cases fail to undergo FGF9-mediated RPE-to-neural retinal transdifferentiation and exhibit the same data profile as Foxg1-Cre; $R x^{\text {flox }}$; FGF9 compound mutants, our characterization and analyses of "Six3-Cre; $R x^{\text {flox}}$; FGF9 compound mutants" in the remaining text refer only to compound mutant samples that exhibit morphological changes. Morphologic analyses of E12.516.5 Nuclear Fast Red-stained sections from Six3-Cre; $R x^{\text {flox }}$; FGF9 compound mutants show that the tissue thickening and loss of pigment phenotype can persist through E16.5 (Fig. 5H-K), but by birth, compound mutant orbits contain only a pigmented cystic remnant (data not shown).

\section{Addition of ectopic FGF9 to the Six3-Cre; $R x$ conditional mutant model causes RPE-to- neural retinal transdifferentiation/respecification.}

Given the morphological changes observed following the ectopic expression of FGF9 in the Six3-Cre; $R x$ conditional mutant background, we sought to characterize the identity of the resulting ocular tissue through immunohistochemical or immunofluorescent staining using known markers for RPE and neural retinal cells. First we examined the expression of Mitf and Otx2, two transcription factors essential for RPE development (Bharti et al., 2006; Bovolenta et al., 1997; Hodgkinson et al., 1993; Martinez-Morales et al., 2003; Nakayama et al., 1998; Nguyen and Arnheiter, 2000; Simeone et al., 1993). At E12.5, Mitf and Otx2 are normally expressed in the RPE (Fig. 6A and E) and are detected throughout the RPE-fated optic vesicle remnant of Six3-Cre; $R x$ conditional mutants (Fig. 6C and G). The FGF9 transgenic model shows a loss of both Mitf and Otx2 staining in the dorsal presumptive RPE region consistent 
with FGF9-induced RPE-to-neural retinal transdifferentiation (Fig. 6B and F). Interestingly, Six3-Cre; $R x^{\text {flox }}$ FGF9 compound mutants show decreased Mitf and Otx2 staining in the two areas of tissue thickening and pigment loss: the distal-dorsal region and the distal-ventral region cupping the developing lens (arrowheads in Fig. 6D and H).

To confirm transdifferentiation of RPE to neural retina via ectopic FGF9 expression in the compound mutant model, we analyzed E12.5 Six3-Cre; $R x^{\text {flox }}$ FGF9 compound mutant and control sections for the expression of known neural retinal cell markers: Chx10 (or Vsx2), Sox2, Pax6, Six3, and CyclinD1 (Burmeister et al., 1996; Grindley et al., 1995; Kamachi et al., 1995; Oliver et al., 1995; Sicinski et al., 1995). In E12.5 sections, neural retinal marker labeling is observed in the presumptive neural retinas of control (arrows in Fig. 7A-E) and OVE1070 (FGF9) animals (Fig. 7F-J) as well as in the RPE-derived neural retinal cells in OVE1070 sections (arrows in Fig. 7F-J), while RPE cells in each model remain unlabeled (white arrowheads in Fig. 7A-J). Consistent with Six3-Cre; $R x$ conditional mutant eyes adopting the RPE fate (Zamora and Mathers, submitted manuscript), no labeling is observed in conditional mutant optic vesicle remnant cells for any of the neural retinal markers tested (Fig. 7K-O). Six3Cre; $R x^{\text {flox }}$ FGF9 compound mutants at E12.5, label for the panel of neural retinal markers, Chx10, Sox2, Pax6, Six3, and CyclinD1, in the areas of tissue thickening that have lost RPE character - the distal-dorsal region (arrows in Fig. 7P-T) and the distal-ventral region (arrowheads in Fig. 7R-T and data not shown) of the presumptive eye. Notably, in compound mutant samples where a distinct lens vesicle is absent or is comparatively undersized, immunolabeling for neural retinal markers is absent in the distal-ventral region of the developing eye (white arrowheads in Fig. 7P-Q and data not shown). Taken together, the loss of RPE markers and the upregulation of neural retinal markers in the compound mutant model suggest 
ectopic expression of FGF9 is sufficient to induce neural retinal transdifferentiation in the RPE cells of Six3-Cre; $R x$ conditional mutants and rescue neural retinal formation, even in the absence of $R x$ expression.

\section{Ectopic FGF9 from the lens can transdifferentiate RPE into neural retina in the $R x$ - inactivated, distal-ventral region of Six3-Cre; $R x^{\text {flox }}$; FGF9 compound mutants.}

To monitor the status of $R x$ activity in the Six3-Cre; $R x^{\text {flox }}$ FGF9 compound mutant model and trace the fate of $R x$-inactivated cells, we utilized the Rosa26-EGFP reporter allele present in our crosses, and performed EGFP reporter analyses via immunostaining with an antiGFP antibody. These analyses allow us to trace the fate of cells that have undergone Six3-Cremediated recombination and, in the case of Six3-Cre; $R x$ conditional mutants and compound mutants, GFP-positive staining marks cells that have lost $R x$ activity through the recombination of the $R x^{\text {flox }}$ allele. In sections from E10.5-12.5 Six3-Cre; OVE1070 (FGF-9) orbits, GFPpositive staining is detected in the neural retina, but not in the RPE or the dorsal RPE-derived neural retina (Fig. 8B, F, J). In Six3-Cre; $R x$ conditional mutants, GFP-positive staining is evident in the distal-ventral region of RPE-fated, optic cup remnants (Fig. 8C, G, K). Interestingly, EGFP reporter analyses in Six3-Cre; $R x^{\text {flox }}$; FGF9 compound mutants show that Cre-mediated recombination occurs only in the distal-ventral portion of the transdifferentiated optic vesicle (arrows in Fig. 8D, H, L), suggesting neural transdifferentiation/respecification in the distal-ventral region can occur in the absence of $R x$ activity. No GFP-positive staining is detected in the distal-dorsal, transdifferentiated portion of Six3-Cre; $R x^{\text {flox }}$; FGF9 compound mutant eyes (arrowheads in Fig. 7D, H, and L).

The Six3-Cre; $R x^{\text {flox }}$; FGF9 compound mutant model is complicated by two potential sources of ectopic FGF9 expression from the OVE1070 (FGF9) transgene: the dorsal RPE and 
the developing lens (Zhao et al., 2001). To monitor the contribution of each potential source of ectopic FGF signaling in these compound mutants and assess whether ectopic FGF signaling in the distal-ventral eye region correlates with the formation of a distinct lens vesicle, we immunofluorescently labeled ocular sections with the pERK antibody. pERK labeling in E11.5

Six3-Cre; $R x^{\text {flox }}$ FGF9 compound mutants reveals consistent staining in the distal-dorsal presumptive eye region (white arrows in Fig. 9D and D'), but only shows pERK-positive staining in the distal-ventral region when a relatively large lens vesicle is present (yellow arrow in Fig. 9D). Conversely, the distal-ventral region is devoid of pERK-positive staining when the lens vesicle is absent or comparatively undersized (yellow arrowhead in Fig. 9D'). These data indicate that ectopic FGF9 expression in the developing lens only reaches levels sufficient for neural retina formation when a relatively large lens vesicle is allowed to form, as opposed to just a lens placode or smaller lens vesicle. Thus, in the Six3-Cre; $R x^{\text {flox }}$ FGF9 compound mutant model, RPE-to-neural retinal transdifferentiation in the distal-ventral region surrounding the lens is dependent on the progression of lens development, which implicates the timing of $R x$ inactivation, and/or the level of ectopic FGF signaling, as key components in establishing the developing eye's competence to undergo FGF-mediated neural retinal specification.

\section{DISCUSSION}

With the creation of compound mutant animals that (a) conditionally inactivate $R x$ in the developing eye during the optic vesicle and optic cup stages, and (b) transgenically express FGF9 in the dorsal RPE and lens, we are able to test whether FGF-mediated neural retinal specification can occur in the absence of $R x$ activity. Our experiments demonstrate that the timing of $R x$ inactivation is a critical factor in determining the developing eye's ability to 
respond to ectopic FGF signaling and ultimately form neural retinal tissue. This is the first demonstration of neural retinal cell specification in $R x$-depleted cells. $R x$-null embryos fail to even form optic vesicles (Mathers et al., 1997), and chimeric embryos composed of wild-type and $R x$-null cells show that $R x$-null cells are excluded from the neural retina (Medina-Martinez et al., 2009).

In Foxg1-Cre; $R x^{\text {flox }}$ FGF9 compound mutants, eye morphology remains unchanged from that of age-matched, Foxg1-Cre; $R x$ conditional mutants, and $R x$-inactivated cells maintain an RPE fate (Fig 1). FGF-driven RPE-to-neural retinal transdifferentiation fails in this compound mutant model (Fig. 4), indicating $R x$ activity is required during the optic vesicle stage to give cells the capacity to undergo FGF-mediated neural retinal specification. The Six3-Cre; $R x$ conditional mutant model has variability in its onset of Cre expression, and thus, $R x$ inactivation, which results in a spectrum of morphological eye phenotypes that consist entirely of RPE-fated cells (Zamora and Mathers, submitted manuscript). For these studies, this variable onset of $R x$ inactivation in the Six3-Cre; $R x$ conditional mutant is used to our advantage when combined with the FGF9 transgene. It allows us to assess the effects of slightly different onsets of Six3-Cremediated $R x$ inactivation in the presence of ectopic FGF signaling, and gives the Six3-Cre; $R x^{\text {flox }}$; FGF9 compound mutant model a broader window of $R x$ inactivation times to compare against the Foxg1-Cre; $R x^{\text {flox }}$; FGF9 compound mutant model.

Our analyses of the Six 3-Cre; $R x^{\text {flox }}$ FGF9 compound mutant model demonstrate that ectopic expression of FGF9 in the Six3-Cre; $R x$ conditional mutant model is able to change the morphology and cellular make-up of the developing eye. Unlike Foxg1-Cre; $R x^{\text {flox }}$; FGF9 compound mutant eyes, the vast majority Six3-Cre; $R x^{\text {flox }}$; FGF9 compound mutant eyes show a loss of RPE identity (Fig. 6) and rescue of neural retinal cell formation (Fig. 7) in the distal-most 
region. Interestingly, among Six 3-Cre; $R x^{\text {flox }}$; FGF9 compound mutants that undergo transdifferentiation, the distal-dorsal region forms neural retina with 100\% consistency (21/21), while neural retinal formation in the distal-ventral region seems to correlate with the formation of a large lens vesicle. These lens vesicles are able to turn on transgenic FGF9 expression and drive FGF levels high enough to drive neural retinal specification, presumably through activation of the $\alpha$ A-crystallin promoter (Lovicu and Overbeek, 1998; Zhao et al., 2001). Data from the EGFP reporter analyses raise the possibility that different mechanisms may be mediating transdifferentiation in the two distal regions: a $R x$-independent mechanism, where $R x$ is inactivated in the distal-ventral region surrounding the lens vesicle yet can still undergo neural retinal specification; and a mechanism in which $R x$ activity is unaltered, and likely activated (Zhao et al., 2001) to mediate RPE-to-neural retinal transdifferentiation in the distal-dorsal region of the developing eye.

Despite the ability of ectopic FGF9 to respecify the neural retinal fate in Six3-Cre; $R x$ conditional mutants with delayed $R x$ inactivation, by E18.5, all Six3-Cre; $R x^{\text {flox }}$; FGF9 compound mutant eyes become a pigmented remnant (data not shown), suggesting $R x$ may play a role in the maintenance of neural retinal identity. Chx10-null mutant mice $\left(o r^{J} / o r^{J}\right)$ show a progressive increase in pigment deposition in the neural retina and a concomitant decrease in the expression of neural retinal markers, indicating $\mathrm{Chx} 10$ also has a role in the maintenance of neural retinal identity (Horsford et al., 2005; Rowan et al., 2004). Thus, while delayed $R x$ inactivation can allow for the initial specification of neural retinal identity via FGF-mediated transdifferentiation, ultimately, the loss of $R x$ may lead to the downregulated expression of neural retinal markers and an inability to maintain neural retinal identity. 
Based on our data, we propose a model in which the duration of $R x$ activity dictates a cell's capacity to assume a neural retinal fate (Fig. 10). We envision a developmental or temporal threshold for $R x$ activity that exists during the optic cup stage: before the threshold, $R x$ is required for FGF-mediated neural retinal specification, but afterwards, neural retinal specification can occur in $R x$-inactivated cells. To make sense of this model, the spatial and temporal patterns of (a) $R x$ activity/inactivation and (b) ectopic FGF9 transgene expression in both compound mutant models must be carefully considered. First, a general comparison of the two compound mutant models shows that ectopic FGF9 expression is unable to rescue neural retinal formation in the Foxg1-Cre; $R x^{\text {flox }}$ FGF9 compound mutant, but is able to rescue neural retinal formation in the Six3-Cre; $R x^{\text {flox }}$; FGF9 compound mutant. The key differences are the temporal onset and spatial domain of Cre recombinase expression and thus, $R x$ inactivation. Foxg1-Cre is expressed in the anterior (nasal) half of the developing optic vesicle beginning at E8.5 (Hebert and McConnell, 2000), inactivating $R x$ in all optic vesicle cells in the anteronasal hemisphere, including the majority of cells in the dorsal optic vesicles of Foxg1-Cre; $R x$ conditional and compound mutants (Fig. 2). Besides having an onset of Cre-mediated recombination that is half a day to a day later than Foxg1-Cre, Six3-Cre expression is restricted to the inner layer of the developing optic cup and the ventral optic stalk, and thus always leaves the presumptive RPE free from Six3-Cre-mediated $R x$ inactivation in the developing eye (Fig 8). Taking into account that the FGF9 transgene is expressed in the dorsal RPE starting at E10.5 (Zhao et al., 2001), in Foxg1-Cre; $R x^{\text {flox}}$; FGF9 compound mutants, FGF9 is expressed in a dorsal RPE domain in which $R x$ has already been inactivated (Figs. 2 and 3). On the other hand, in Six3-Cre; $R x^{\text {flox }}$; FGF9 compound mutants, FGF9 is expressed in the distal-dorsal RPE domain (Fig. 9) in which $R x$ has not been inactivated (Fig. 8), and can thus be specified for the 
neural retinal fate (Fig. 7). This is supported by the fact that the distal-dorsal RPE domain is converted to the neural retinal fate in nearly all Six3-Cre; $R x^{\text {flox }}$ FGF9 compound mutants analyzed (21/24). Based on these observations, it appears $R x$ activity dictates the ability of presumptive RPE cells to respond to FGF-mediated neural retinal specification/transdifferentiation.

Considering the distal-ventral region of Six3-Cre; $R x^{\text {flox }}$; FGF9 compound mutant eyes, interpretation of the model is somewhat complicated by its ability to sometimes specify neural retinal identity in the distal-ventral region, which, by the time FGF9 expression is turned on, has already undergone Six3-Cre-mediated $R x$ inactivation. Interestingly, we demonstrate neural retinal identity in the distal-ventral region correlates with the formation of a lens vesicle that is able to serve as another source of ectopic FGF9 expression (Figs. 6 and 9). The FGF9 transgene is driven by a lens-specific $\alpha$ A-crystallin promoter (Lovicu and Overbeek, 1998; Zhao et al., 2001), and its additional expression in the lens vesicle seems to be a key factor in establishing neural retinal cell identity in the $R x$-inactivated, distal-ventral region of Six3-Cre; $R x^{\text {flox }}$; FGF9 compound mutant eyes. Previous characterization of Six3-Cre; $R x$ conditional mutants shows that the formation of lens vesicle is dependent on delayed $R x$ inactivation and prolonged $R x$ mediated retinal progenitor cell proliferation effects (Zamora and Mathers, submitted manuscript). However, neural retinal specification was never observed in Six3-Cre; $R x$ conditional mutants. Taken together, these data suggest that the timing of $R x$ inactivation is the critical factor in this complex, compound mutant model. Delayed inactivation prolongs $R x$ mediated proliferation, allows lens vesicle formation, and thus controls activation of the $\alpha \mathrm{A}-$ crystallin promoter-driven FGF9 transgene (Lovicu and Overbeek, 1998; Zhao et al., 2001). Secondarily, given developing compound mutant eyes with smaller lens vesicles do not show 
distal-ventral conversion to the neural retinal fate, the level of ectopic FGF expression may also play a role in specifying neural retinal identity in $R x$ mutant cells.

In addition to promoting FGF9 expression from the developing lens, delayed $R x$ inactivation may also allow these distal-ventral cells to pass a temporal or developmental competence threshold, and give $R x$-inactivated cells the ability to respond to ectopic FGF9 emanating from the lens vesicle, and promote neural retinal fate determination. Our data supports a model where $R x$, prior to its delayed Six3-Cre-mediated inactivation, could activate a network of genes that gives cells the ability to undergo FGF-mediated neural retinal specification in the absence of $R x$ activity. Further research must be conducted to identify candidate genes and networks, and confirm this possibility. Although highly unlikely, we cannot rule out the possibility that following $R x$ inactivation, residual Rx protein is not completely turned over and is able to exert its neural retinal specification effects in response to ectopic FGF9 expression.

Our results indicate the duration of $R x$ activity is critical in FGF-mediated neural retinal specification, and support a time-dependent function for $R x$ in establishing optic vesicle cell competence to specify the neural retinal cell fate. Overall, our work suggests a developmental or temporal $R x$ activity threshold exists during the optic cup stage, after which neural retinal cells may be specified in the absence of $R x$. 


\section{ACKNOWLEDGEMENTS}

We would like to thank Dr. Mark Lewandoski for the Foxg1-Cre transgenic mouse strain; Dr. Guillermo Oliver for the Six3-Cre transgenic mouse strain and anti-Six3 antibody; Dr. Paul Overbeek for the OVE1070 (FGF9) mouse strain and SV40 pA plasmid; Drs. Visvanathan Ramamurthy and Heinz Arnheiter for the gifts of reagents; and Dennis Cole for expert technical assistance. The Pax6 monoclonal antibody developed by A. Kawakami was obtained from the Developmental Studies Hybridoma Bank developed under the auspices of the NICHD and maintained by The University of Iowa, Department of Biology, Iowa City, IA. This research was supported by EY012152. 


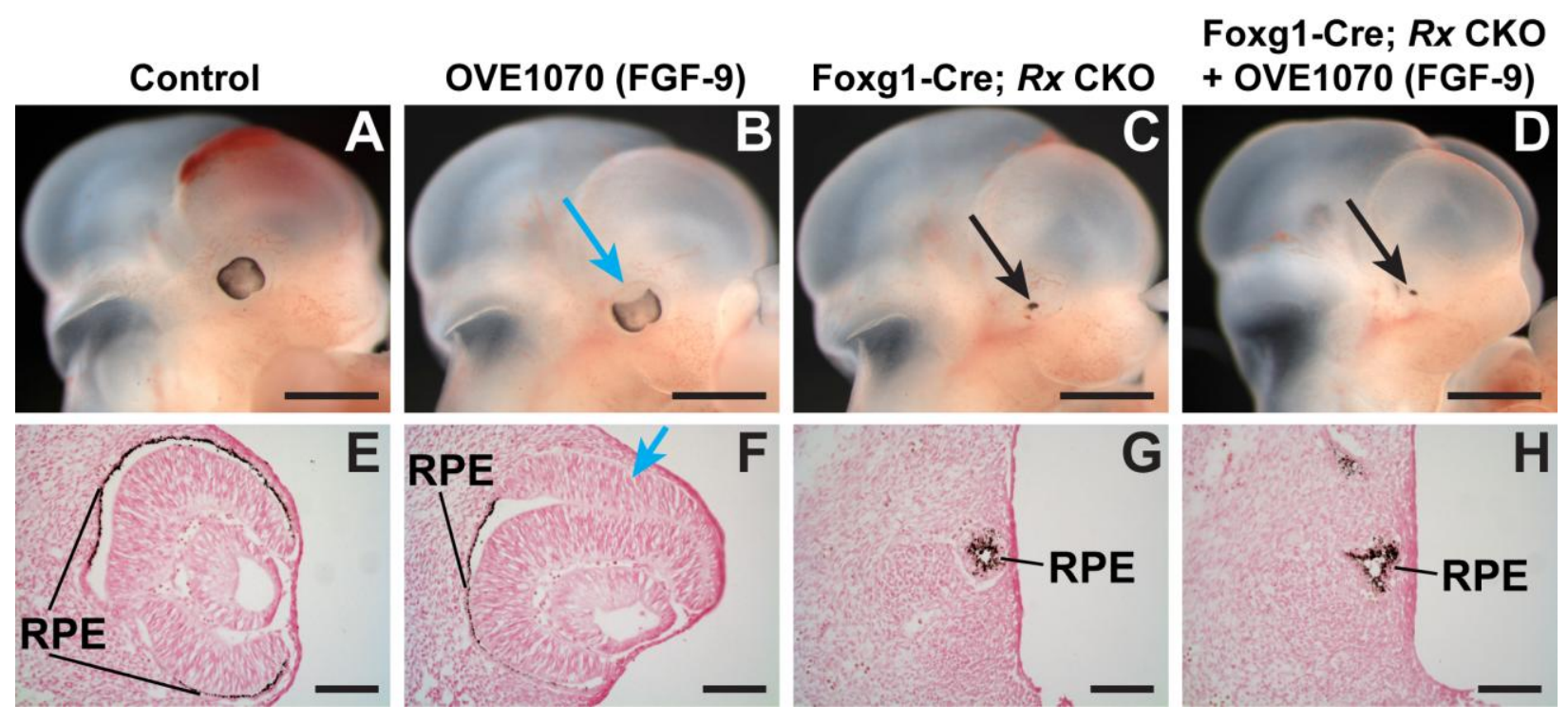

Figure 1. Ectopic FGF signaling fails to rescue ocular morphology of the Foxg1-Cre; $R x$ conditional mutant model. (A-D) Whole-mount E11.5 heads. (E-H) Nuclear Fast Redstained coronal sections through E11.5 orbits. Expression of FGF9 in the dorsal RPE of Foxg1-Cre; $R x$ conditional mutant model $(\mathrm{D}$ and $\mathrm{H}$ ) maintains gross and morphologic phenotype of Foxg1-Cre; $R x$ conditional mutant ( $\mathrm{C}$ and $\mathrm{G})$-an optic vesicle remnant completely consisting of RPE-fated cells. Blue arrows indicate RPE-to-neural retinal transdifferentiation in the OVE1070 (FGF9) transgenic model. RPE, retinal pigment epithelium. Scale bars: A-D, $500 \mu \mathrm{m} ; \mathrm{E}-\mathrm{H}, 100 \mu \mathrm{m}$. 


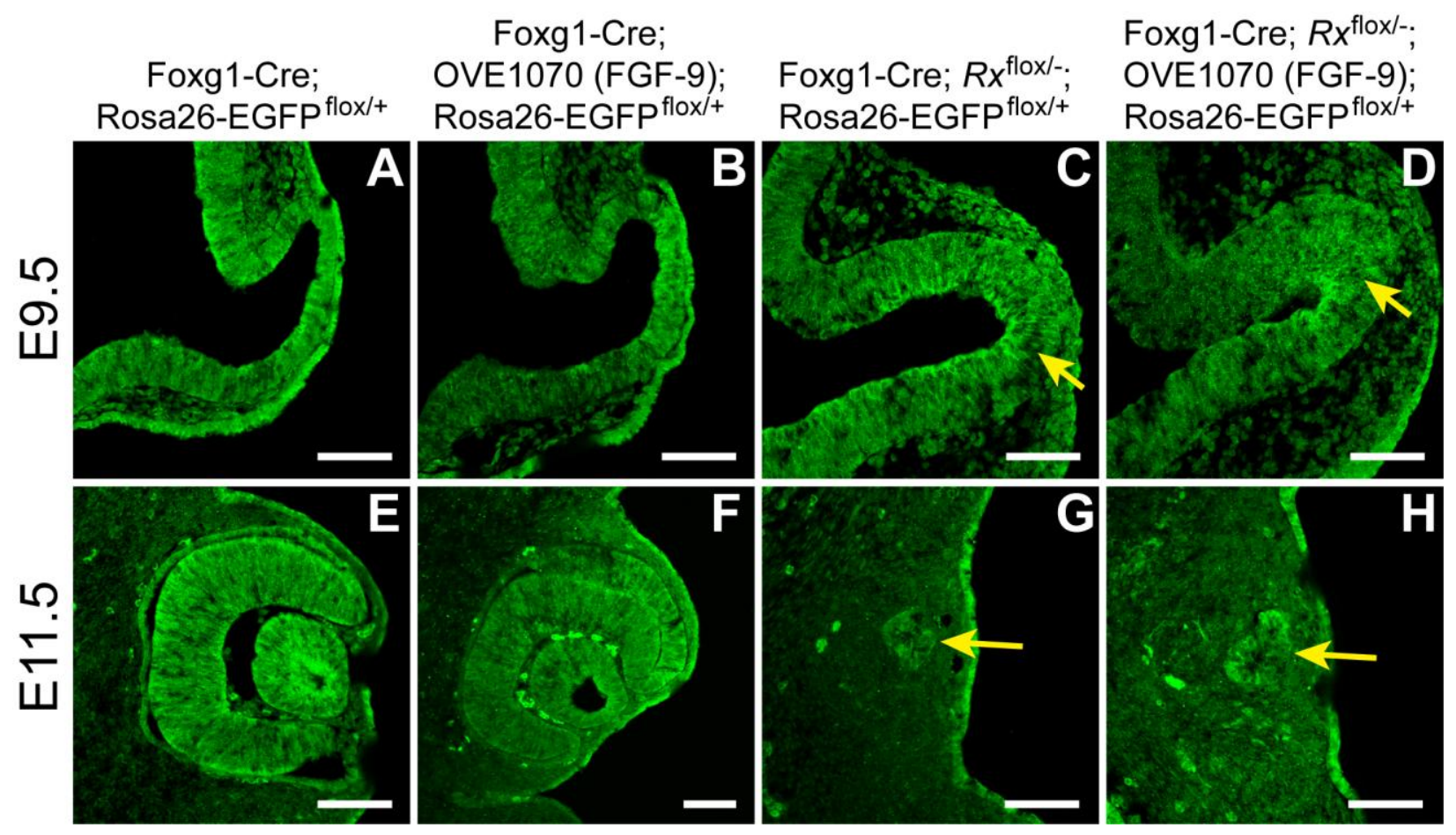

Figure 2. Cells of the $R x$-deleted lineage remain present in the optic remnants of Foxg1-Cre; $R x^{\text {flox; }}$ FGF9 compound mutants. (A-H) Rosa26-EGFP reporter activity is assayed by immunofluorescent staining with an anti-GFP antibody on coronal sections through the orbit of Foxg1-Cre; $R x^{\text {flox; }}$ FGF9 compound mutants and controls at the stages indicated in the left column. The Rosa26-EGFP reporter is activated throughout the developing optic vesicle in Foxg1-Cre-positive E9.5 samples (A-D) and remains activated in all ocular structures at E11.5 (E-H). Arrows indicate staining in optic vesicle remnants of Foxg1-Cre; $R x$ conditional mutants and compound mutants. Scale bars: $100 \mu \mathrm{m}$. 


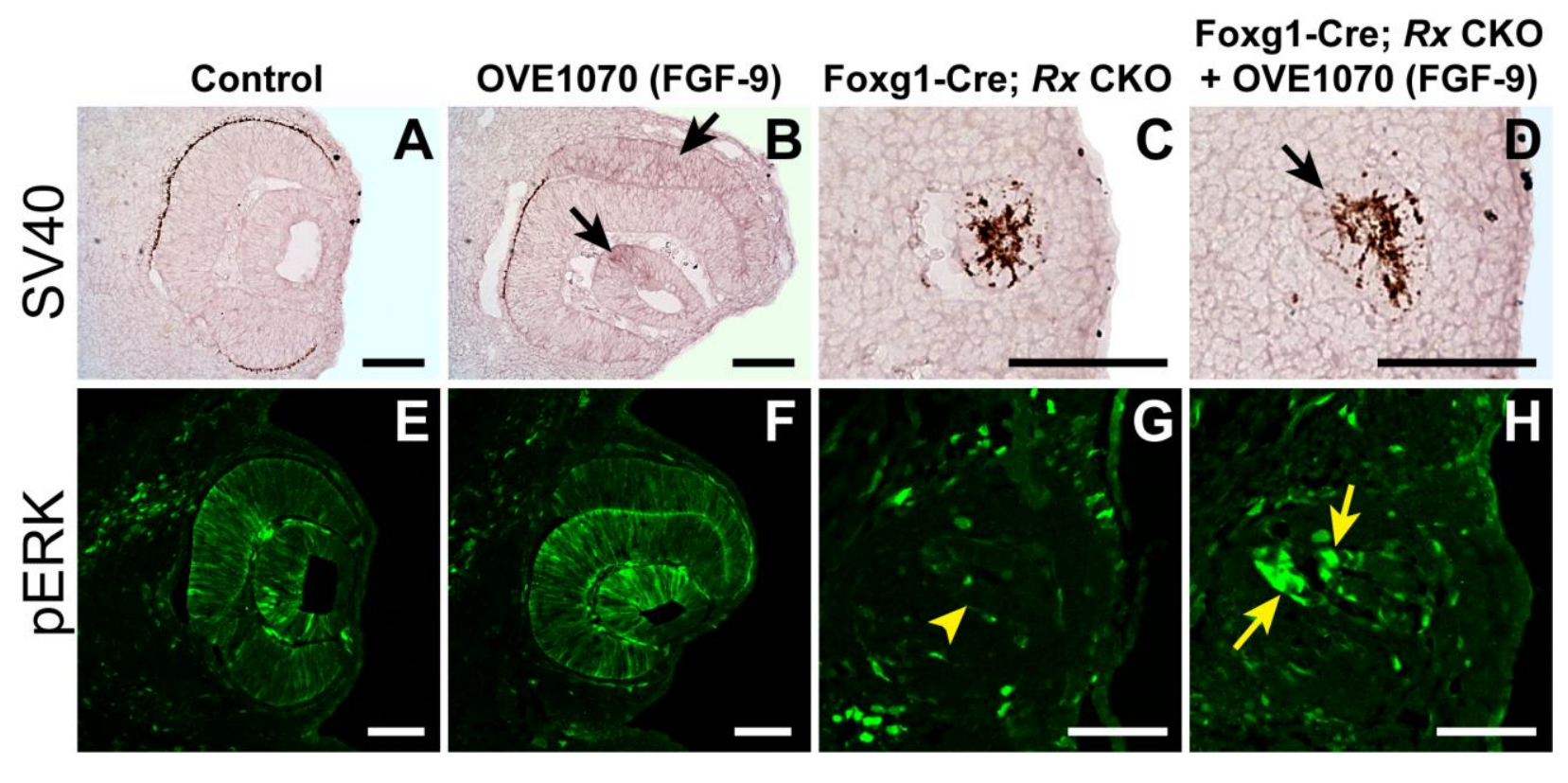

Figure 3. Ectopic FGF signaling (MEK/ERK pathway) via the OVE1070 transgene is

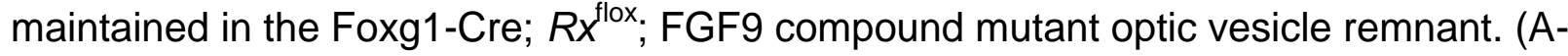

D) Transgenic expression of FGF9 is detected by in situ hybridization using an antisense SV40 riboprobe on E11.5 coronal sections through the orbit of Foxg1-Cre; $R x^{\text {flox; }}$ FGF9 compound mutants and controls. At E11.5, The FGF9 single mutant transgenically expresses FGF9 in the dorsal RPE region and the posterior lens vesicle (arrows in B). (E-H) Immunofluorescent staining for phosphorylated ERK1/2 (pERK) on coronal sections through the orbit of E11.5 Six3-Cre; $R x^{\text {flox; }}$ FGF9 compound mutants and controls. No pERK-positive labeling is detected in the optic tissue of Foxg1-Cre; $R x$ conditional mutant samples (arrowhead in $\mathrm{G}$ ), but is present in optic vesicle remnant of

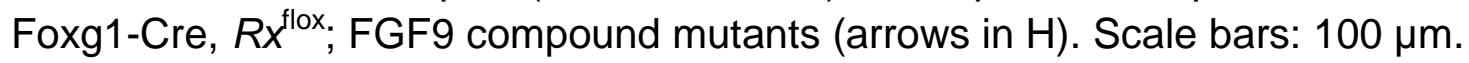




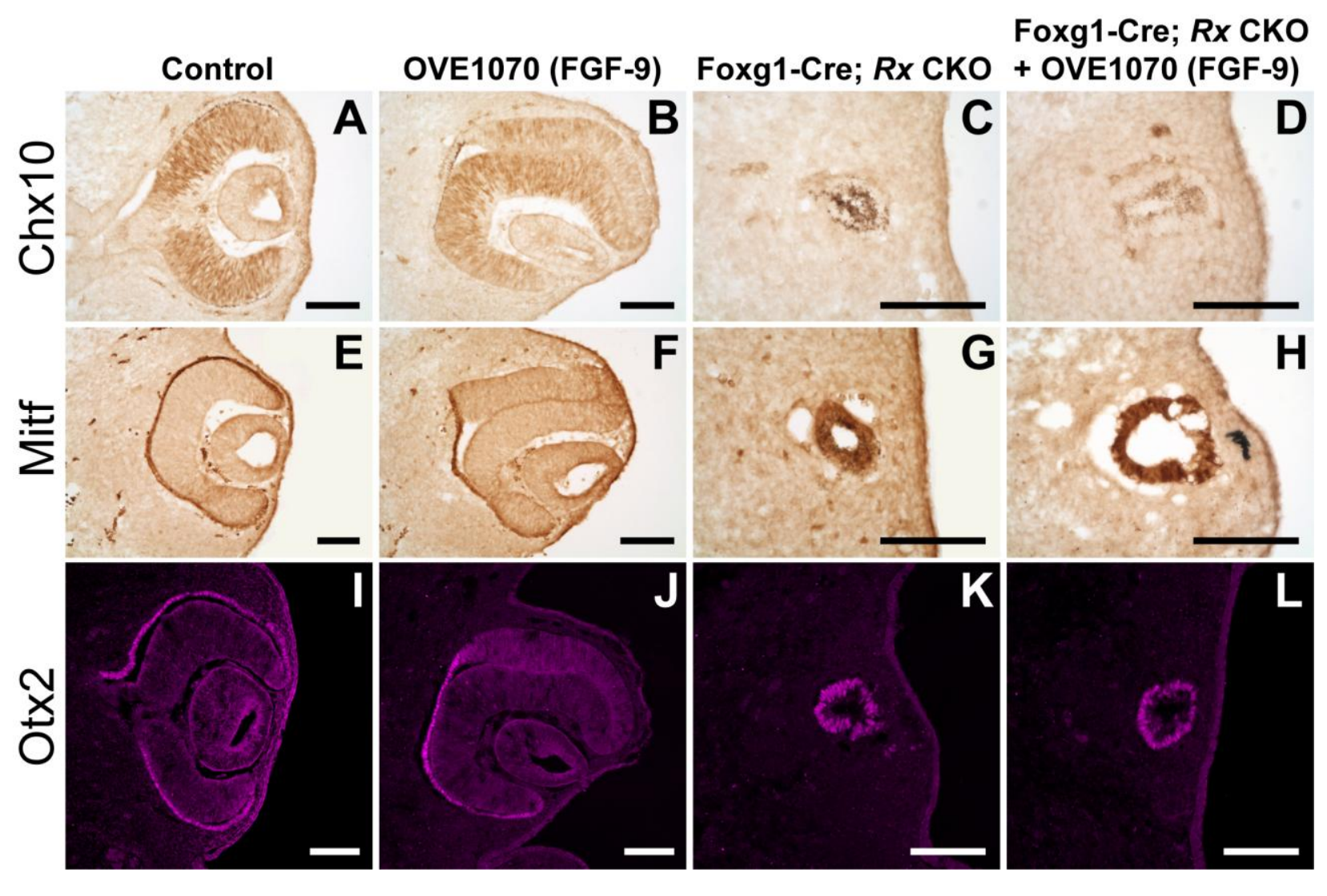

Figure 4. Ectopic FGF signaling fails to rescue neural retinal specification in Foxg1-Cre; $R x$ conditional mutants, and the compound mutant optic vesicle remnant maintains an RPE fate. (A-L) Immunostaining for neural retinal cell marker, Chx10 (A-D), and RPE markers, Mitf (E-H) and Otx2 (I-L), on E11.5 coronal sections through the orbit of Foxg1-Cre, $R x^{\text {flox }}$; FGF9 compound mutants and controls. Chx10 staining is absent from

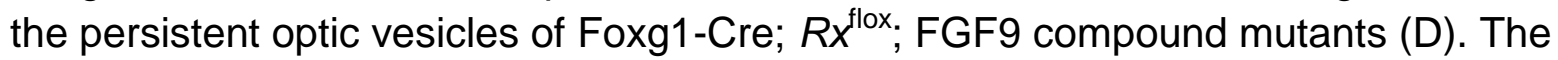
persistent optic vesicles of compound mutants stain throughout for Mitf and Otx2 $\mathrm{CH}$ and $\mathrm{L}$ ), indicating no change from the RPE cell fate of Six3-Cre; $R x$ conditional mutants, following the introduction of ectopic FGF9 signaling. Scale bars: $100 \mu \mathrm{m}$. 


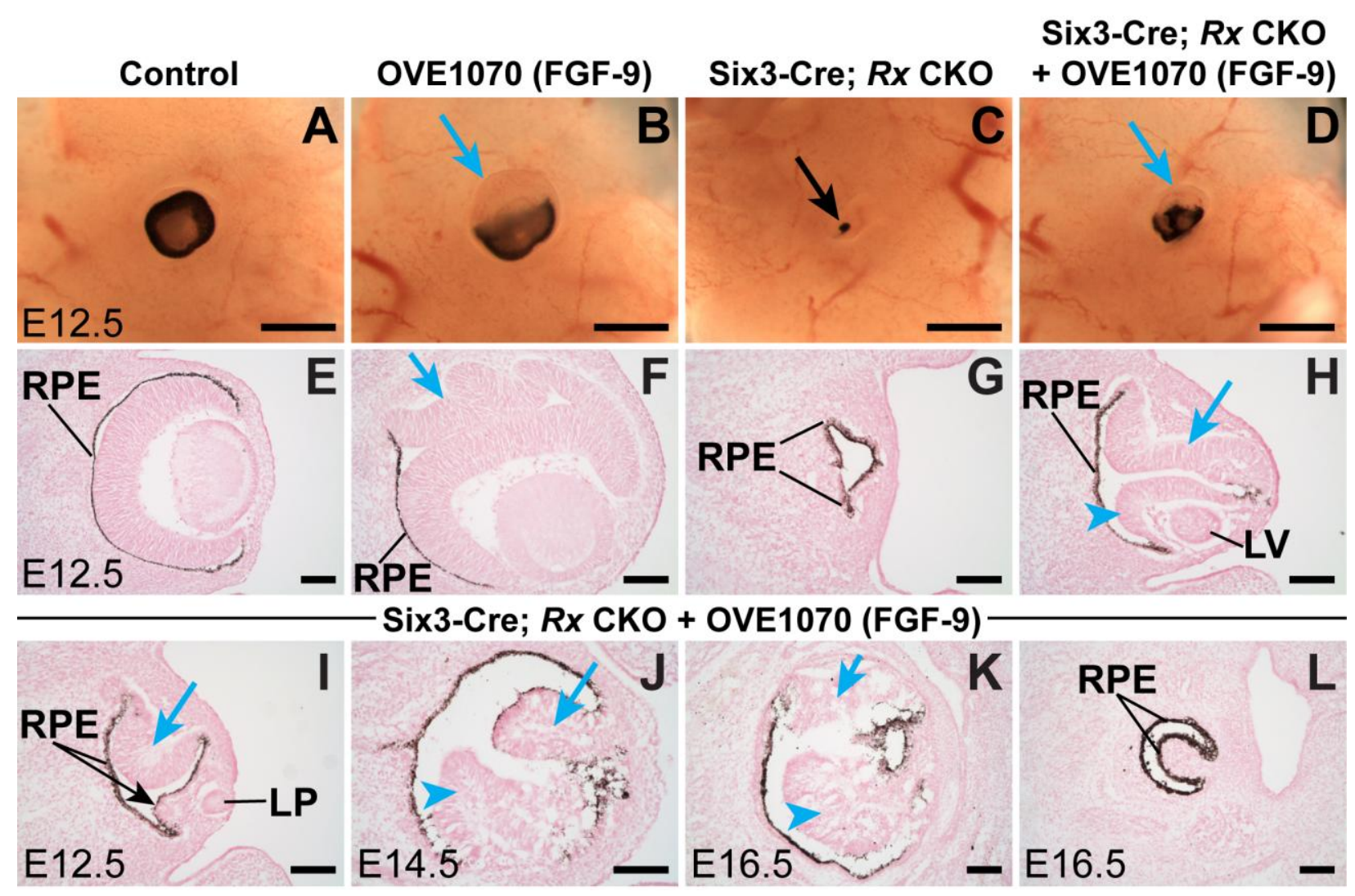

Figure 5. Addition of ectopic FGF signaling to Six3-Cre; $R x$ conditional mutant partially rescues developing eye morphology. (A-D) Whole-mount E12.5 orbits. Ectopic, nonpigmented retinal tissue forms in the dorsal portion of eye with the addition of the OVE1070 (FGF-9) transgene to Six3-Cre; $R x$ conditional mutant (arrow in D). (E-I) Nuclear fast red-stained coronal sections through E12.5 Six3-Cre; $R x^{\text {flox; }}$ FGF9 compound mutant (both $\mathrm{H}$ and $\mathrm{I}$ ) and control orbits (E-G). Ectopic FGF9 expression converts the completely pigmented ocular tissue of the Six3-Cre; Rx conditional mutant (G) to a thickened, non-pigmented tissue in two distinct, distal eye locations-the distaldorsal region (blue arrows in $\mathrm{H}-\mathrm{K}$ ); and the distal-ventral region surrounding the lens (blue arrowheads in $\mathrm{H}-\mathrm{K}$ ) -and partially rescues retinal morphology. When lens development fails to reach the lens vesicle stage, the distal-ventral region does not transdifferentiate from the RPE fate in Six3-Cre; $R x^{\text {flox }}$; FGF9 compound mutants (black arrow in $\mathrm{H}^{\prime}$ ). (I-K) Nuclear fast red-stained coronal sections through the orbit of Six3Cre; $R x^{\text {flox }}$ FGF9 compound mutants at the indicated stages. RPE, retinal pigment epithelium; LV, lens vesicle; LP, lens placode. Scale bars: A-D, $500 \mu \mathrm{m}$; E-L, $100 \mu \mathrm{m}$. 


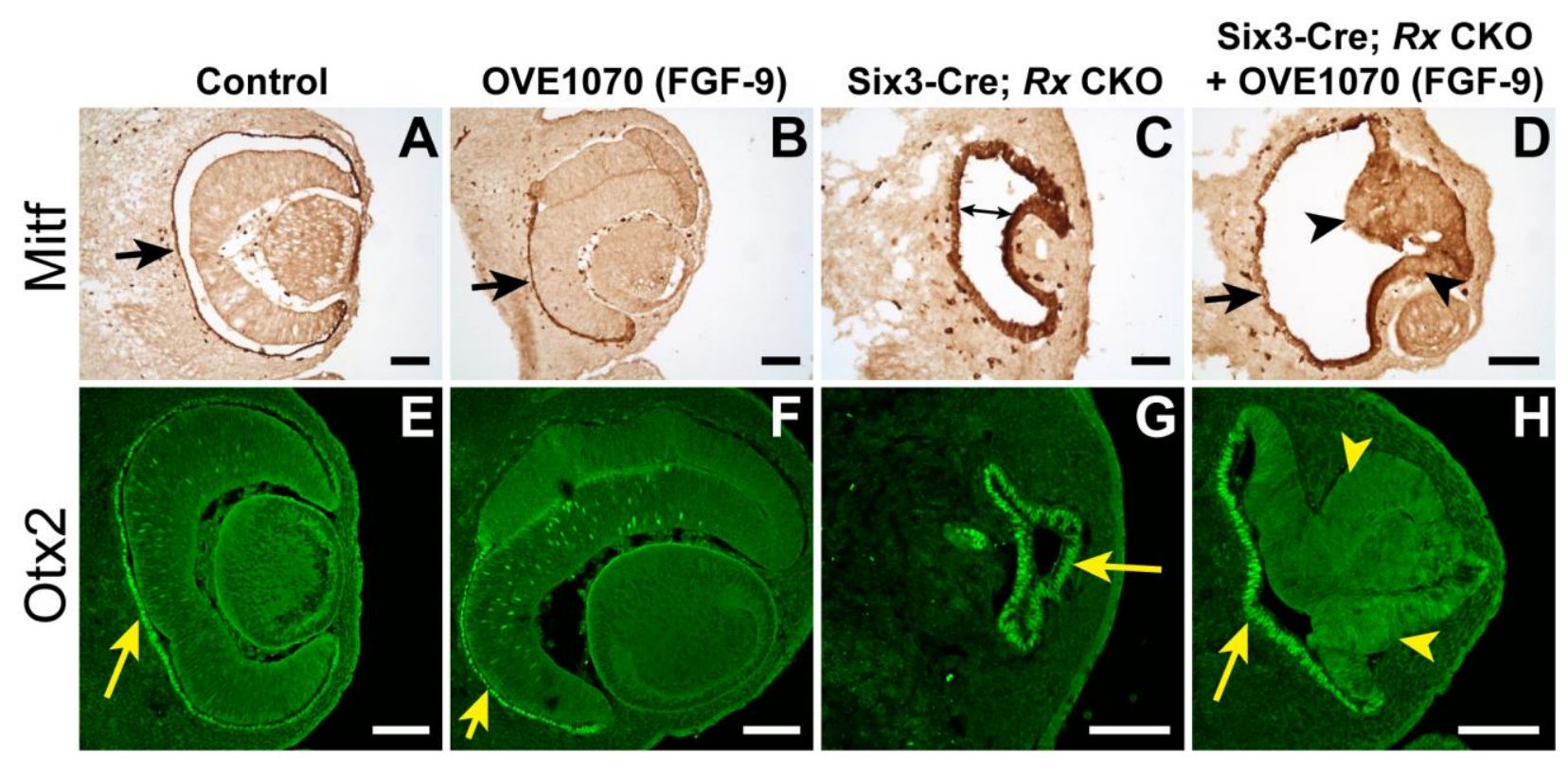

Figure 6. RPE identity of the Six3-Cre; $R x$ conditional single mutant eye is lost with the introduction of ectopic FGF signaling (OVE1070 transgene). (A-H) Immunostaining for RPE markers, Mitf and Otx2, on E12.5 coronal sections through the orbit of Six3-Cre, $R x^{\text {flox; }}$ FGF9 compound mutants and controls. RPE marker immunostaining is decreased in the thickened, non-pigmented distal regions of Six3-Cre; $R x^{\text {flox; }}$ FGF9 compound mutant ocular tissue (arrowheads in $\mathrm{D}$ and $\mathrm{H}$ ), in comparison to Six3-Cre; $R x$ conditional single mutant, which immunostains throughout the ocular tissue for Mitf and Otx2 (arrows in C and G). The Six3-Cre; $R x^{\text {flox; }}$ FGF9 compound mutant phenotype shows Mitf and Otx2 immunostaining patterns comparable to that of the OVE1070 (FGF9) single mutant control (B and F), in which Mitf and Otx2 staining is lost where the dorsal RPE transdifferentiates to neural retinal tissue, and similarly indicates a change of fate from RPE for the Six3-Cre; $R x^{\text {flox; }}$ FGF9 compound mutant. Arrows highlight areas of positive immunolabeling for the indicated RPE marker. Arrowheads indicate areas of decreased immunolabeling. Scale bars: $100 \mu \mathrm{m}$. 


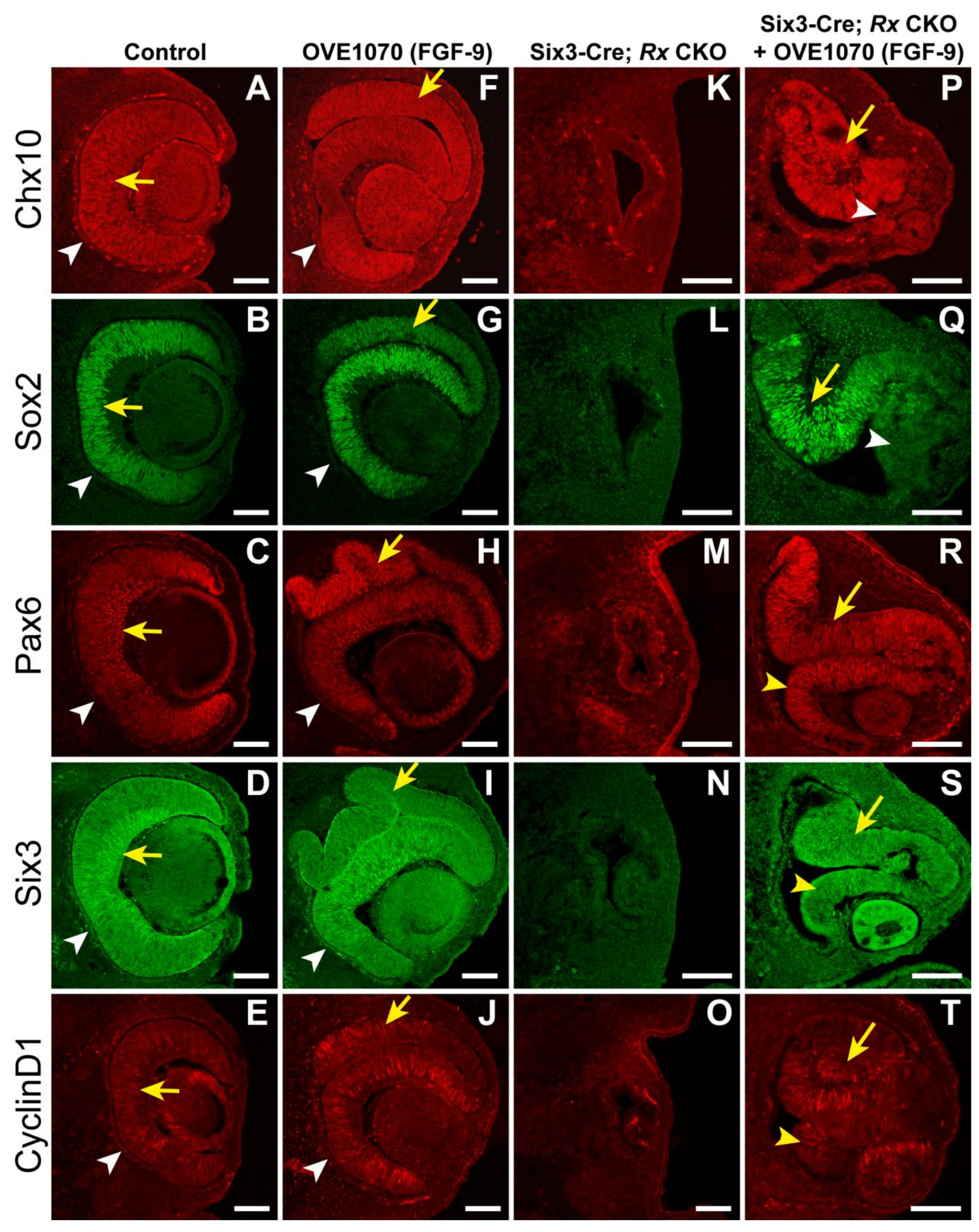


Figure 7. Neural retinal cell fate is respecified with the addition of ectopic FGF9 signaling to the Six3-Cre; $R x$ conditional mutant model. (A-T) Immunofluorescent labeling for neural retinal cell markers (as indicated in the left margin of each row) on coronal sections through the orbits of E12.5 Six3-Cre; $R x^{\text {flox; }}$ FGF9 compound mutants and controls. The OVE1070 (FGF-9) single mutant control shows positive neural retinal cell marker labeling in the primary neural retina as well as the dorsal RPE-derived transdifferentiated neural retina (arrows in F-J) and no labeling in the undifferentiated RPE (white arrowheads in F-J). Six3-Cre; $R x$ conditional mutant sections show no neural retinal cell marker labeling in their resultant optic vesicle remnants $(\mathrm{K}-\mathrm{O})$. Neural retinal cell marker labeling is observed in two thickened, non-pigmented, distal areas in

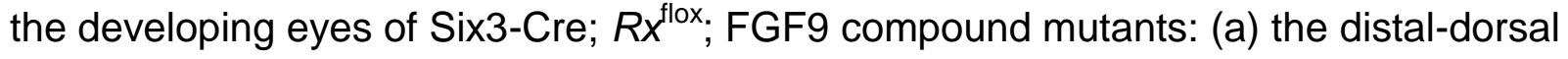
region (arrows in $\mathrm{P}-\mathrm{T}$ ) and (b) the distal-ventral region surrounding the lens (arrowheads in R-T). Yellow arrows and arrowheads highlight areas of positive immunofluorescent labeling for the indicated neural retinal marker. White arrowheads demarcate areas that lack immunofluorescent labeling for the indicated neural retinal marker, i.e. untransdifferentiated RPE. Scale bars: $100 \mu \mathrm{m}$. 


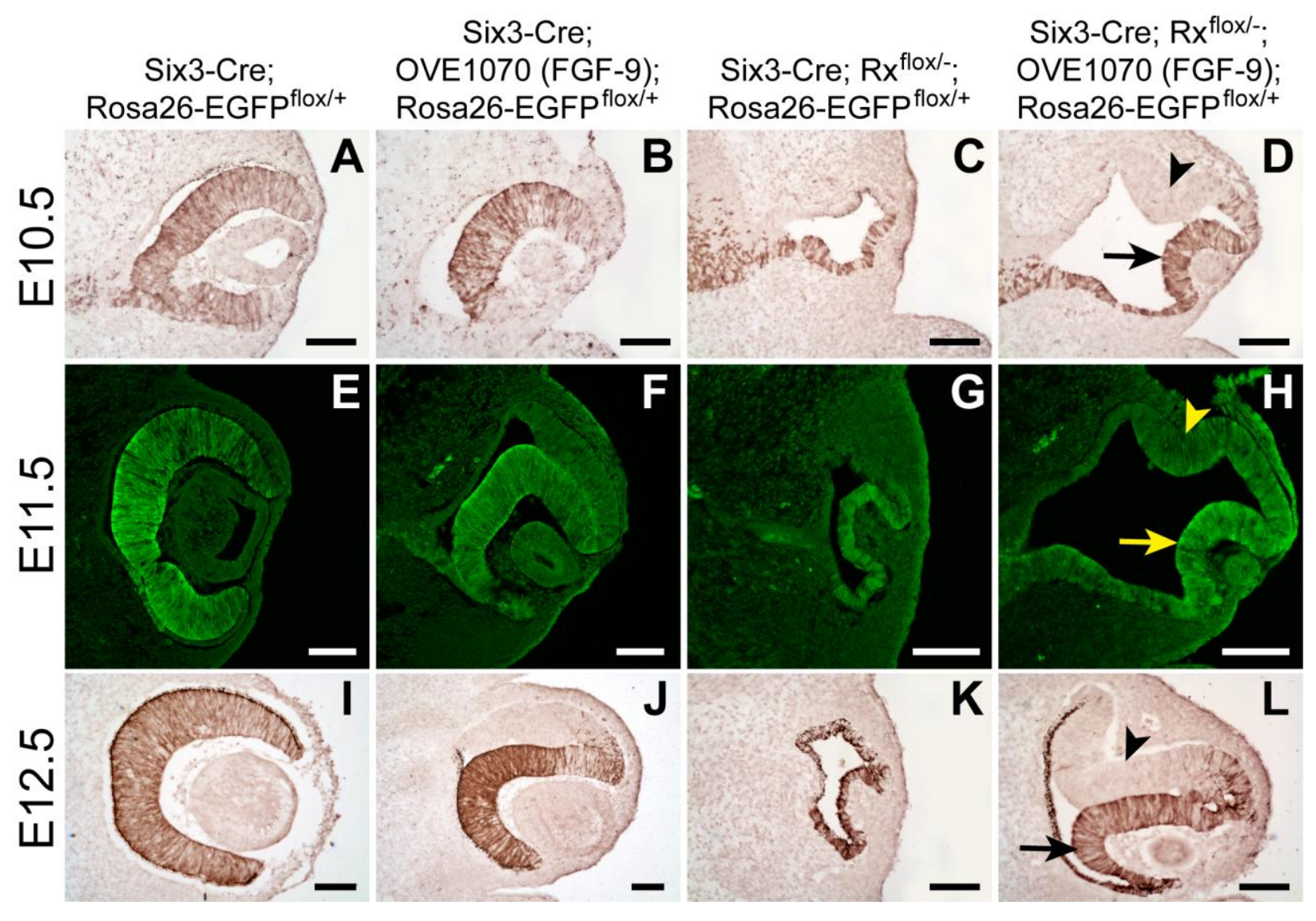

Figure 8. FGF-mediated neural retinal respecification can occur in the absence of $R x$ activity. (A-L) Rosa26-EGFP reporter activity assayed by immunostaining (A-D and I-L) or immunofluorescence $(\mathrm{E}-\mathrm{H})$ with an anti-GFP antibody on coronal sections through

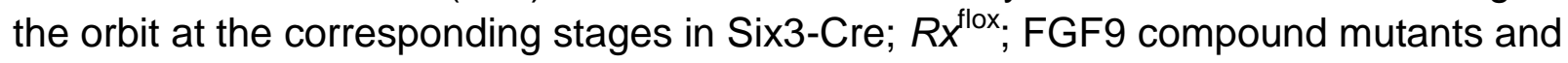
controls. The Rosa26-EGFP reporter is activated in the distal-ventral respecified neural retinal tissue adjacent to the lens (arrow in D, $H$, and L) of Six3-Cre $R x^{\text {flox; }}$ FGF9 compound mutants, but not the distal-dorsal region (arrowhead in $\mathrm{D}, \mathrm{H}$, and $\mathrm{L}$ ), indicating Six3-Cre-mediated $R x$ inactivation has occurred in the cells of the distalventral respecified neural retina. Scale bars: $100 \mu \mathrm{m}$. 


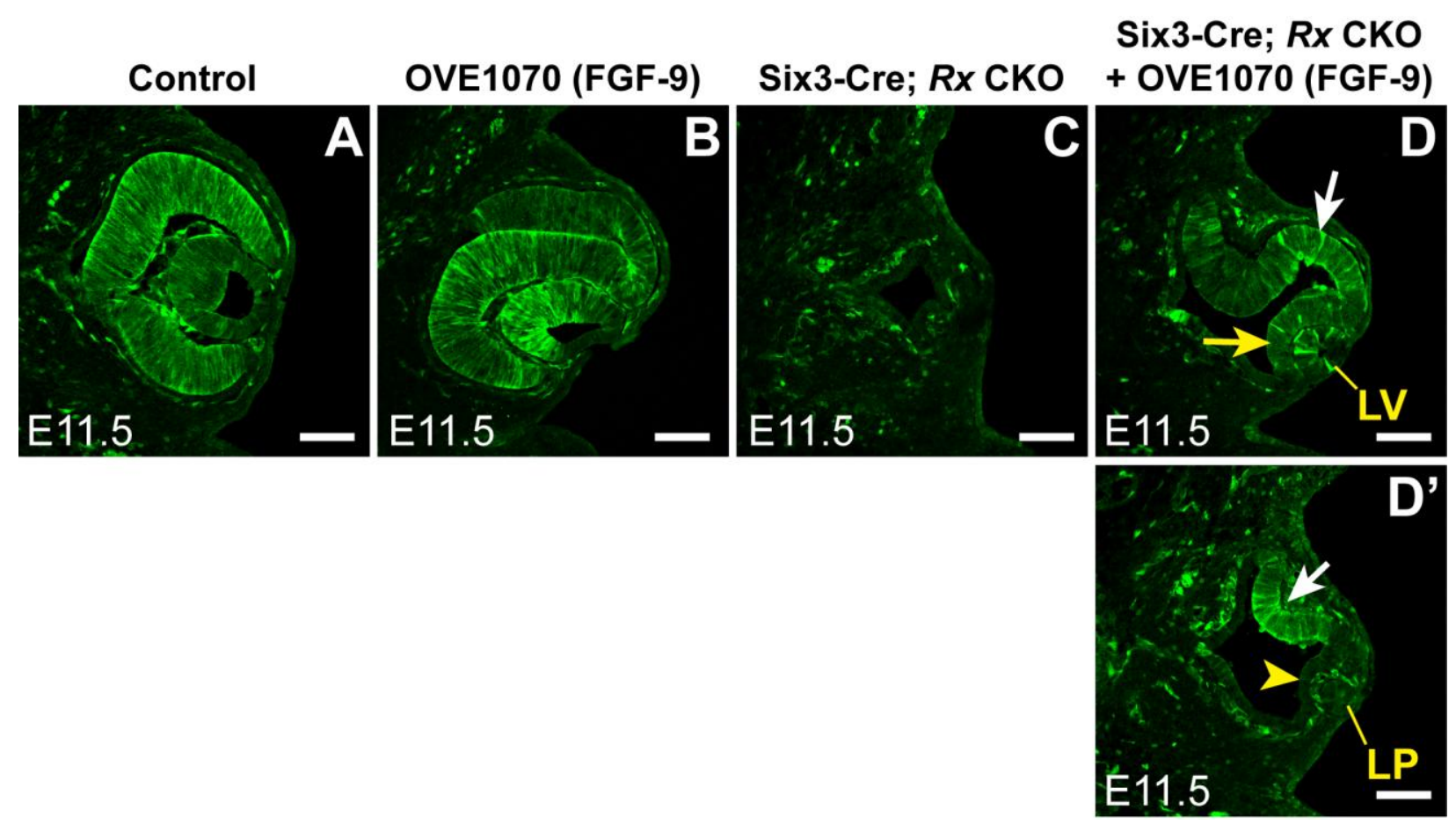

Figure 9. Neural retinal respecification in the distal-ventral, $R x$-depleted region surrounding the developing lens correlates with lens vesicle-dependent FGF9 signaling and ERK activation. (A-D') Immunofluorescent staining for phosphorylated ERK1/2 (pERK) on coronal sections through the orbit of E11.5 Six3-Cre; $R x^{\text {flox; }}$ FGF9 compound mutants and controls. In Six3-Cre; $R x^{\text {flox; }}$ FGF9 compound mutants, ERK activation and RPE-to-neural retinal transdifferentiation only occur in the distal-ventral, $R x$-depleted region when a lens vesicle develops (compare arrow in D to arrowhead in D'). LV, lens vesicle; LP, lens placode. Arrows indicate areas of pERK-positive staining. Arrowhead indicates area where no pERK-positive staining is detected. Scale bars: $100 \mu \mathrm{m}$. 
Threshold for $R x$-independent neural retinal specification?

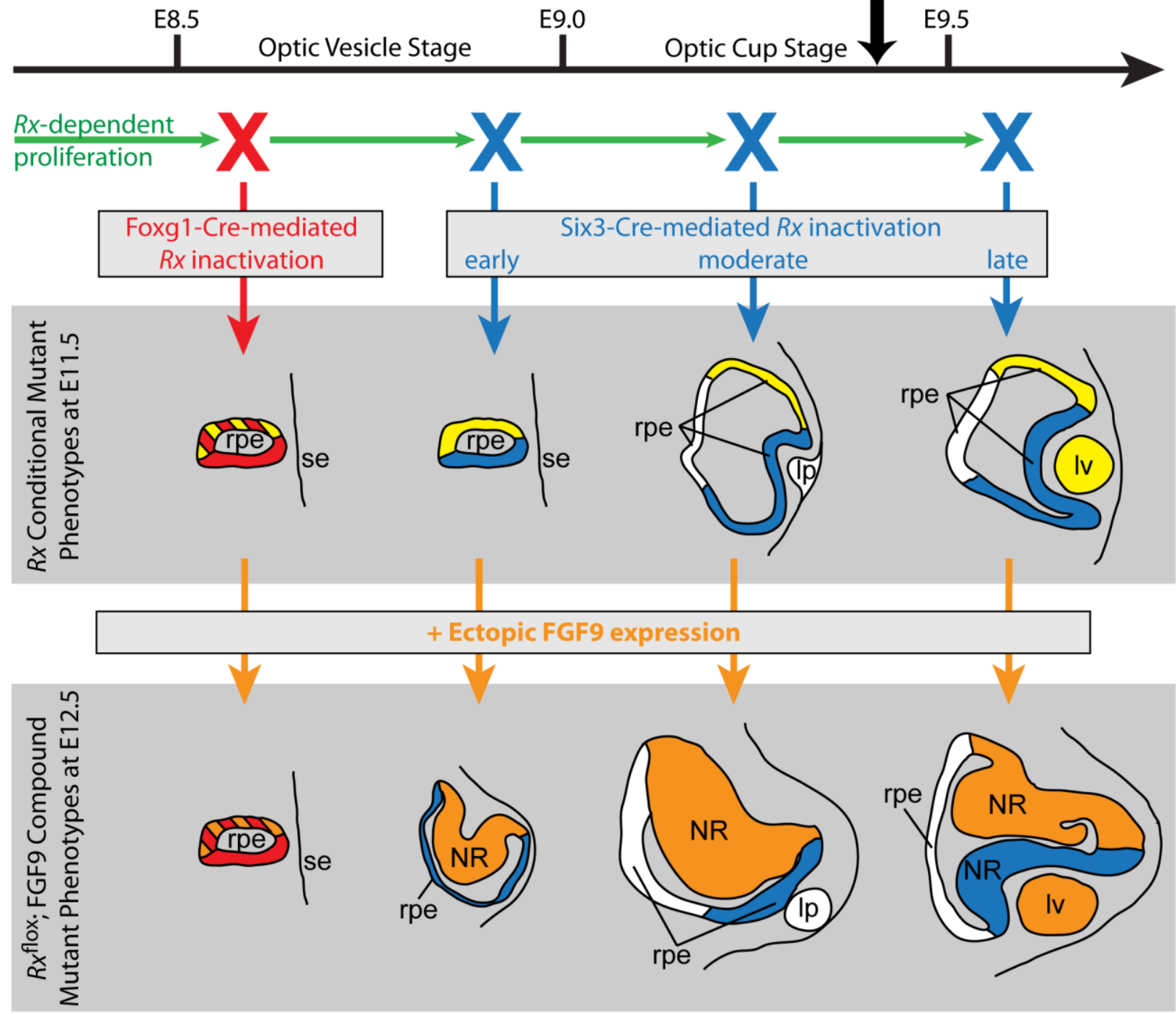
$R$-inactivated domain (Foxg1-Cre)
$\square$ FGF9 transgene expression-competent tissue
$\square R x$-inactivated domain (Six3-Cre)
$\square$ FGF9 transgene expressing domain 
Figure 10. Model for phenotypic outcomes of Foxg1-Cre; $R x^{\text {flox; }}$ FGF9 (red) and Six3-Cre; $R x^{\text {flox; }}$; FGF9 compound mutants (blue). Foxg1-Cre-mediated inactivation of Rx during the optic vesicle stage results in an optic vesicle remnant composed entirely of $R x$-inactivated RPE cells (red). Addition of FGF9 transgene to this $R x$ conditional mutant model cannot rescue neural retinal formation in Foxg1-Cre; $R x^{\text {flox; }}$ FGF9 compound mutant tissue because $R x$ activity is required at this early stage. Variable onset of Six3-Cre expression from the late optic vesicle through optic cup stage restricts $R x$ inactivation to the distal-ventral domain of the resulting optic structure and generates a spectrum of phenotypes dependent on the precise timing of $R x$ inactivation. In the distal-dorsal region of Six3-Cre; $R x$ conditional mutants, the $R x^{\text {flox }}$ allele does not undergo Six3Cre-mediated recombination (no blue shading), therefore with the addition of ectopic FGF9, $R x$ activity remains unaltered and can participate in FGF-induced neural retinal specification (NR in orange). In the $R x$-inactivated, distal-ventral region of Six3-Cre; $R x^{\text {flox; }}$ FGF9 compound mutant eyes, FGF9-mediated neural retinal specification is dependent on the progression of lens vesicle development, which represents the crossing of a developmental threshold, after which (a) FGF9 transgenic expression can be turned on via the $\alpha$ A-crystallin promoter (Iv in orange) and (b) neural retinal specification, in response to ectopic FGF expression, can occur independent of $R x$ activity (NR in blue). rpe, retinal pigment epithelium; se, surface ectoderm; Ip, lens placode; Iv, lens vesicle; NR, neural retina induced by ectopic FGF9 expression. 
Chapter IV: Summary, Implications, and Future Directions 
The work presented in this dissertation provides additional insight into the functions of $R x$ in early vertebrate eye and retinal development and has uncovered a novel role for $R x$ in FGFmediated RPE-to-neural retinal transdifferentiation. Much of the work describing the functions of $R x$ have either focused on the earlier aspects of $R x$ expression-its roles in eye/retinal field specification and proliferation, as well as optic vesicle evagaination —or focused on the study of $R x$ mutations associated with developmental defects or disease. The studies described in this dissertation were specifically designed to ascertain the functions of $R x$ following optic vesicle evagination, a developmental period where the exact functions of $R x$ have not been completely defined. Through the use of our conditional $R x$ alleles, in conjunction with Cre-expressing and other transgenic animal models, we have spatially and temporally manipulated $R x$ activity and generated conditional and compound mutants to tease out the functions of $R x$ in early vertebrate ocular development. The results presented here support a role for $R x$ in retinal progenitor cell (RPC) proliferation, optic cup and lens morphogenesis, and neural retinal specification, and also indicate $R x$ activity dictates competence to undergo FGF-mediated RPE-to-neural retinal transdifferentiation.

Two models of $R x$ conditional inactivation, both inactivating $R x$ after optic vesicle evagination, demonstrate $R x$ is required for normal proliferation of RPCs. $R x$ inactivation severely stunts overall eye and retinal growth and morphogenesis, with delayed inactivation allowing for progressively more growth and morphogenesis in the developing eye. Inactivation of $R x$ during the optic vesicle stage suspends retinal development at the optic vesicle stage and completely blocks the invagination and formation of an optic cup. Inactivation of $R x$ during optic cup development allows for the initiation of optic cup morphogenesis, indicating delayed $R x$ 
inactivation (or prolonged $R x$ activity) maintains RPC proliferation long enough to support the beginnings of optic cup invagination.

Alternatively, the effects on optic cup morphogenesis could be due to $R x$-mediated changes in neural retinal progenitor cell migration. in vivo, time-lapse confocal microscopy studies in eyeless medaka mutants, which are Rx3-null mutants that fail to evaginate optic vesicles (Loosli et al., 2001; Winkler et al., 2000), indicate that $R x 3$ is cell-autonomously required for retinal progenitor cells to converge and then migrate to drive optic vesicle evagination from the anterior neural plate (Rembold et al., 2006). Thus, it may be possible that mouse $R x$ has a similar function in the migration and invagination of neural retinal progenitors during optic cup formation — delayed $R x$ inactivation during optic cup development may allow for the initiation of neural retinal progenitor cell migration and the initial steps of optic cup morphogenesis. In order to test whether mouse $R x$ carries this function, conditional $R x$ inactivated cells could be labeled through the introduction of a tdTomato reporter allele, and tracked in $R x$ conditional mutant embryo cultures via time-lapse confocal microscopy. The biggest obstacle would be developing culture conditions that would sustain embryonic development and developing ocular morphology over a $~ 2$ day span, from E9.0-E11.0, during which, the complete process of optic cup morphogenesis could be fully observed.

Interestingly, our results show that the role of $R x$ in RPC proliferation not only has an impact on overall eye and retinal morphogenesis, but also influences lens development in the surface ectoderm. Inactivation of $R x$ in the developing optic vesicle allows contact to be made between the optic vesicle and the surface ectoderm, yet thickening of the lens placode is completely blocked. Delayed inactivation of $R x$ during optic cup development allows lens induction to initiate, as surface ectoderm morphology and lens marker analyses are indicative of 
early lens formation. This is interesting in light of the fact that $R x$ is not expressed in the surface ectoderm or developing lens, and suggests $R x$ may influence lens induction through the activation of signaling molecules from the developing optic vesicle/cup. Indeed, in situ hybridizations from Foxg1-Cre; $R x$ conditional mutants show downregulated expression of extrinsic signaling molecules known to be involved in lens induction-Bmp4, Fgf8 and Fgfl5within the $R x$-depleted optic vesicle (Voronina et al., unpublished manuscript). Thus, delayed $R x$ inactivation, during optic cup development, may likewise delay downregulation of $B m p 4, F g f 8$, and Fgfl5 and provide a mechanism by which lens induction is initiated in mild and moderate Six3-Cre; $R x$ conditional mutants.

Despite the changes in developing eye morphology allowed by conditional $R x$ inactivation, the cellular identity of $R x$-inactivated cells remains uniform $-R x$ inactivation during both optic vesicle and optic cup development completely blocks neural retinal formationdemonstrating the importance of $R x$ in specifying neural retinal cell identity. These conclusions are corroborated by a report of mouse embryonic chimeras consisting of wild-type and $\mathrm{Rx}^{-/-}$ mutant cells, which shows $R x$-deficient cells are excluded from the developing neural retina (Medina-Martinez et al., 2009). And similar to our severely affected Six3-Cre; $R x$ conditional mutants and Foxg1-Cre; $R x$ conditional mutants, chimeras made up of a majority of $R x^{-{ }^{-}}$cells have a pigmented optic vesicle remnant phenotype.

Finally, results from the Six3-Cre; $R x^{\text {flox}}$; FGF9 compound mutant model demonstrate a novel function for $R x$ in FGF-mediated RPE-to-NR transdifferentiation. Somewhat surprisingly, we found that overexpression of FGF9 is able to specify neural retinal identity in $R x$-depleted cells, but cannot maintain this identity through embryonic development. We propose a model in which $R x$ activity is absolutely required to specify neural retinal cell identity until a temporal or 
developmental threshold is met during the optic cup stage; after this threshold is met, neural retinal specification can be induced by FGF signaling, independent of $R x$ activity. This model is based on the fact that FGF9 overexpression is only able to rescue neural retinal identity in $R x$ depleted cells when Six3-Cre-mediated $R x$ inactivation is delayed enough to generate a mild conditional mutant phenotype. Thus, the timing of $R x$ inactivation is a key factor in determining a cell's competence to undergo FGF-induced transdifferentiation. Secondarily, in this compound mutant model, activation of FGF9 expression in the developing lens vesicle may also contribute to the transdifferentiation of $R x$-depleted cells, but it too is dependent on prolonged $R x$ activity. The generation of a lens vesicle that is developed enough to activate the $\alpha \mathrm{A}$-crystallin promoterdriven FGF9 transgene requires prolonged $R x$ activity to promote proliferation and the progression of lens vesicle formation. Thus, regardless of the mechanism, the temporal component of $R x$ activity in this model plays a central role in FGF-mediated neural retinal specification.

While our studies have elucidated some of the functions of $R x$ during optic vesicle and optic cup development, many of the downstream targets of $R x$ that mediate these effects remain unidentified. Therefore, a potential future experiment could be microarray analyses to identify genes that are differentially regulated in the conditional and compound $R x$ mutants versus wildtype controls. We could ask questions such as, "What genes/networks/pathways are regulated by $R x$ that allow it to affect RPC proliferation, neural retinal specification, optic cup morphogenesis, lens induction, cell migration, etc.?" and gain insight at the whole genome level.

In more general terms, this work has implications outside of the field of eye development. The retina is a very specialized extension of the brain, and like the brain, its cells are highly organized into a laminar pattern. Because of its similar organization and because the population 
of neuronal cell types is less diverse than that in the brain, the retina has long served as a model for understanding the development, structure and function of neuronal tissues and networks, which can then be used to gain insight into similar aspects of the more complex brain. As such, the study of how a transcription factor, like $R x$, directs organogenesis and the specification of a neural fate versus a non-neural fate may have applications in understanding how similar development and fate decisions are made in the brain. Furthermore, $R x$ is expressed in the developing ventral hypothalamus and posterior pituitary regions of the brain. Thus many of the strategies employed here to study eye development could be used to understand the role of $R x$ in the development of those brain structures.

In studying the functions of $R x$ in early ocular development, we gain insights into the processes and interactions required for normal organogenesis of the eye. The knowledge gained through these types of studies thus have the potential to be translated and applied toward the development of replacement and/or regenerative therapies to treat ocular and retinal diseases. With the advent of embryonic stem and induced pluripotent cell research, these possibilities are getting ever closer to becoming reality. Thus, an understanding of $R x$ 's role in specifying, promoting, and maintaining the proliferation of retinal progenitor cells could have implications for generating retinal tissue to replace diseased or damaged tissue. In fact, a proof-of-principle study in mouse embryonic stem (ES) cells showed that ectopic expression of $R x$ is able to direct their differentiation into retinal cells (Tabata et al., 2004). The study also reported that $R x$ induced ES cells were able to migrate into host retinal explants and express ganglion and horizontal cell markers, while uninduced ES cells were unable to do either. This indicates more specialized progenitors that are closer in identity to cells of the host tissue may have enhanced ability to be integrated into the host tissue than pluripotent stem cells. Studies such as this one as 
well as those described in this dissertation provide the basic science foundation from which translational, replacement and regenerative therapies will be generated to help patients suffering from debilitating ocular diseases. 
Chapter V: Appendix 
Based on our data that suggest $R x$ is required for neural retinal specification, we also wanted to test whether $R x$ is sufficient to specify neural retina. We hypothesized that if $R x$ is sufficient to specify neural retina, then its ectopic expression in the developing retinal pigment epithelium (RPE) and optic stalk would drive the transdifferentiation of those tissues into neural retina. To specifically test this hypothesis, we designed a $6.0 \mathrm{~kb}$ transgenic construct, Trp2FLAG-hRX, that would ectopically drive $R x$ in the developing RPE and optic stalk (Fig. 1A). This construct contains an $\mathrm{N}$-terminal, FLAG epitope-tagged version of the human $R X$ gene, driven by a $1.7 \mathrm{~kb}$ fragment of the Trp2 (tyrosinase-related protein 2) promoter. Trp2 (or Dct; dopachrome tautomerase) is one of the earliest molecular markers for retinal pigment epithelium (RPE) and melanocyte differentiation. During mouse embryogenesis, $\operatorname{Trp} 2$ is first expressed in the optic vesicle at E9.5 (Steel et al., 1992). The $1.7 \mathrm{~kb} \operatorname{Trp} 2$ promoter fragment used in this construct has been shown to drive expression in the developing RPE and optic stalk of mouse embryos, beginning at E9.5 (Zhao and Overbeek, 1999).

Trp2-FLAG-hRX transgenic animals were generated by injecting the $6.0 \mathrm{~kb}$ transgenic construct into blastocysts and implanting those blastocysts into pseudo-pregnant, foster mother mice. Four founder animals (Trp2-470, Trp2- 483, Trp22-466, and Trp2-478) were generated in the WVU Transgenic Core Facility, but showed no obvious eye phenotype. Each founder was backcrossed to a wild-type FVB mouse to propagate each transgenic strain. Trp2-478 was euthanized due to erratic behavior and an inability to breed. Trp2-483 was euthanized following 4 consecutive litters of pups that did not carry the transgene. The remaining founders, Trp2-470 and Trp2-466, were able to transmit the Trp2-FLAG-hRX transgene to their offspring, although at a rate below the expected 50\%. One of the first litters produced from the pairing of Trp2-470 and a WT FVB female gave off one Trp2-FLAG-hRX transgenic animal with a microphthalmic 
eye phenotype (\#590). Following this, we aggressively mated both Founder Trp2-470 and his microphthalmic offspring, \#590, in an effort to generate more microphthalmic animals. Interestingly, the pairings from this strain transmitted the Trp2-FLAG-hRX transgene at rate of about $42 \%$ (45/107), and 30 of those 45 transgenics were microphthalmic (Fig. 1B). Founder line Trp2-466 transmitted the transgene, but never gave any interesting eye phenotypes.

Because the FVB strain carries a retinal degeneration allele, $P d e 6 b^{r d l}$, we reasoned that backcrossing our transgenic animals, which were generated in the FVB strain, to FVB wild-types may be causing the microphthalmic phenotype. Thus, we crossed our transgenic lines into the C57BL/6 background strain. Transgenic animals that consistently gave off microphthalmic offspring never transmitted the microphthalmic phenotype when crossed into the C57BL/6 background. These data suggest that generation of the Trp2-470 transgenic strain probably caused an insertional mutation that either interacts with the $P d e 6 b^{r d l}$ mutation or some other locus in the inbred FVB strain to cause microphthalmia.

More importantly, analyses of Trp2-470 embryos show that early eye development is not altered in these transgenic animals - in particular, transdifferentiation of the RPE or optic stalk is never observed. Western analyses of Trp2-470 embryonic eyes using an anti-FLAG M2 antibody (Stratagene) indicate that the transgenic protein product is expressed and is of the appropriate molecular weight, $\sim 45 \mathrm{kDa}$ (Fig. 1C). Co-Immunofluorescent assays, using the anti-FLAG M2 and anti-Mitf antibodies on cryosections through the orbits of transgenic embryos, show that the Trp2-FLAG-hRX transgene is ectopically expressed in the RPE and optic stalk, as expected (Fig. 1D). However, careful inspection of these immunofluorescent-stained sections reveals FLAG$\mathrm{hRX}$ is excluded from the nucleus of RPE and optic stalk cells. Thus, this transgenic animal model prevents us from directly testing our hypothesis because, in this model, $\mathrm{hRX}$ is never 
given the chance to exert its effects in the nucleus. If $\mathrm{hRX}$ (a paired-like homeodomain transcription factor) were able to promote the transdifferentiation of RPE and/or optic stalk into neural retina, it would most likely do so through the transcriptional activation of genes required for neural retinal determination. But the inability to localize $h R X$ into the nucleus prevents its ability to mediate changes in transcriptional activity and ultimately precludes this transgenic model from being used to test whether $R x$ is sufficient to specify the neural retinal fate. It is not completely understood why FLAG-hRX is excluded from the nucleus.

Figure 1. The Trp2-FLAG$\mathrm{hRX}$ transgenic animal model

(A) Schematic of the Trp2FLAG-hRX injection fragment used to produce transgenic animals. Arrows indicate primers for PCR genotyping.

(B) Microphthalmic phenotype in Trp2-470 line when crossed to FVB background.

(C) Western blot using antiFLAG M2 antibody, showing protein product of transgenic expression is of the appropriate molecular weight, 45 $\mathrm{kDa}$.

(D) Immunofluorescent staining of E11.5 Trp2470 eye section showing Trp2-FLAG-hRX is appropriately expressed in the RPE, but is excluded from the nucleus.

A

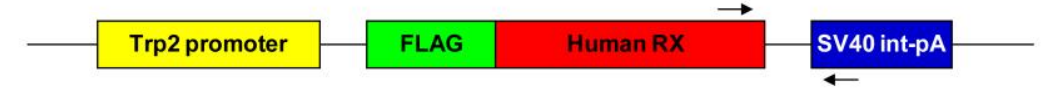

B

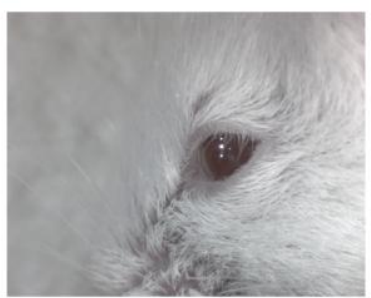

Trp2-470 \#593

(normal phenotype)

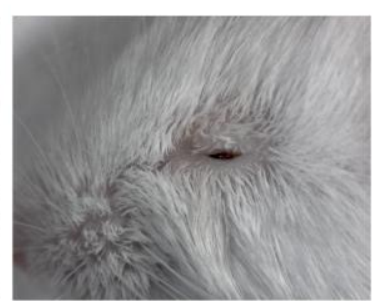

Trp2-470 \#792

(microphthalmic)

C

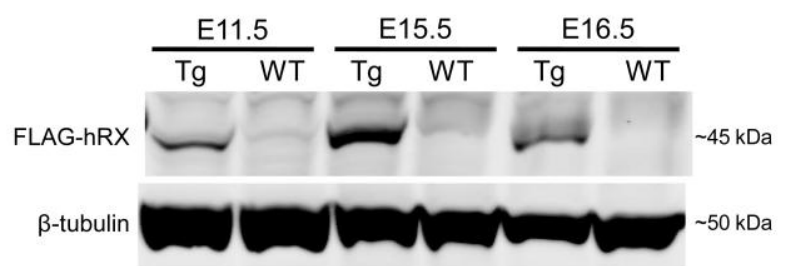

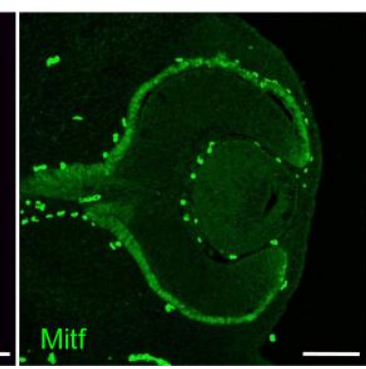

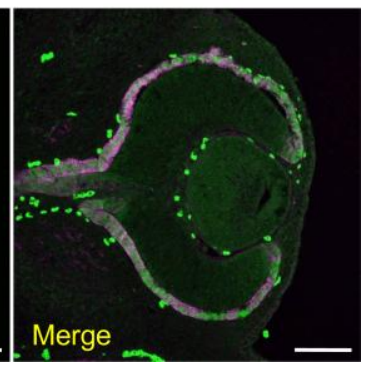




\section{Materials and Methods:}

Generation of Trp2-FLAG-hRX transgenic animals. Trp2-FGF9 vector was given to us by the Overbeek lab. The Trp2 promoter fragment was excised and then inserted into the Not1 site of the pCMV-Tag2b-FLAG-hRX vector to create the pCMV-Tag2b-Trp2-FLAG-hRX vector. A 6.0 kb Trp2-FLAG-hRX transgenic injection fragment was generated from the pCMV-Tag2bTrp2-FLAG-hRX vector via restriction endonuclease digestion with Sac1 and Mlu1. The transgenic injection fragment was purified and injected into blastocysts, which were then implanted into pseudopregnant CD-1 mothers by the WVU Transgenic Animal Core Facility. Founders were genotyped using the following primers: Trp2F, 5 'GTCTGAAAGCCAAGGAGCA-3'; and Trp2R, 5'-AAATGAGCCTTGGGACTGTG-3'. Western Analysis. E11.5 forebrains and E15.5 and E16.5 eyes were dissected and flash frozen on dry ice. Tissue was homogenized by sonication in Urea Sample Buffer (200 $\mu \mathrm{L}$ for brains, $100 \mathrm{uL}$ for eyes; USB = 6M urea, 4\% SDS, $125 \mathrm{mM}$ Tris-HCl, bromophenol blue dye). Samples were diluted (1:5 for FLAG M2 Ab samples, and 1:20 for beta-tubulin Ab samples), then run on $10 \%$ Tris- $\mathrm{HCl}$ polyacrylamide gel at $120 \mathrm{~V}$ for 1.25 hours. Separated proteins were transferred to PVDF membrane at $250 \mathrm{~mA}$, then probed with Stratagene anti-FLAG M2 antibody (1:500, mouse). Loading control was anti- $\beta$-tubulin antibody (1:2000, mouse). Primary antibodies were incubated for 1 hour at room temperature. Secondary antibody was Anti-mouse IgG, $680 \mathrm{~nm}$ (1:50,000); incubated for 45 minutes at room temperature. Membrane staining was visualized by Li-Cor Odyssey.

Tissue Preparation and Immunohistology. Embryonic heads were fixed in 4\% Paraformaldehyde overnight at $4^{\circ} \mathrm{C}$, cryoprotected in $30 \%$ sucrose in PBS, and cryo-sectioned, 
coronally, at 12 microns. Primary antibodies used were FLAG M2 (1:1000; Stratagene) and Mitf (1:1000; gift from Arnheiter lab). 
Chapter V: References 
Abremski, K., Hoess, R., Sternberg, N., 1983. Studies on the properties of P1 site-specific recombination: evidence for topologically unlinked products following recombination. Cell. 32, 1301-11.

Adler, R., Canto-Soler, M. V., 2007. Molecular mechanisms of optic vesicle development: complexities, ambiguities and controversies. Dev Biol. 305, 1-13.

Amae, S., Fuse, N., Yasumoto, K., Sato, S., Yajima, I., Yamamoto, H., Udono, T., Durlu, Y. K., Tamai, M., Takahashi, K., Shibahara, S., 1998. Identification of a novel isoform of microphthalmiaassociated transcription factor that is enriched in retinal pigment epithelium. Biochem Biophys Res Commun. 247, 710-5.

Andreazzoli, M., Gestri, G., Angeloni, D., Menna, E., Barsacchi, G., 1999. Role of Xrx1 in Xenopus eye and anterior brain development. Development. 126, 2451-60.

Andreazzoli, M., Gestri, G., Cremisi, F., Casarosa, S., Dawid, I. B., Barsacchi, G., 2003. Xrx1 controls proliferation and neurogenesis in Xenopus anterior neural plate. Development. 130, 5143-54.

Ashery-Padan, R., Marquardt, T., Zhou, X., Gruss, P., 2000. Pax6 activity in the lens primordium is required for lens formation and for correct placement of a single retina in the eye. Genes Dev. 14, 2701-11.

Avilion, A. A., Nicolis, S. K., Pevny, L. H., Perez, L., Vivian, N., Lovell-Badge, R., 2003. Multipotent cell lineages in early mouse development depend on SOX2 function. Genes Dev. 17, 126-40.

Azuma, N., Tadokoro, K., Asaka, A., Yamada, M., Yamaguchi, Y., Handa, H., Matsushima, S., Watanabe, T., Kida, Y., Ogura, T., Torii, M., Shimamura, K., Nakafuku, M., 2005.

Transdifferentiation of the retinal pigment epithelia to the neural retina by transfer of the Pax 6 transcriptional factor. Hum Mol Genet. 14, 1059-68.

Bailey, T. J., El-Hodiri, H., Zhang, L., Shah, R., Mathers, P. H., Jamrich, M., 2004. Regulation of vertebrate eye development by Rx genes. Int J Dev Biol. 48, 761-70.

Bao, Z. Z., Cepko, C. L., 1997. The expression and function of Notch pathway genes in the developing rat eye. J Neurosci. 17, 1425-34.

Barton, K. M., Levine, E. M., 2008. Expression patterns and cell cycle profiles of PCNA, MCM6, cyclin D1, cyclin A2, cyclin B1, and phosphorylated histone H3 in the developing mouse retina. Dev Dyn. 237, 672-82.

Baumer, N., Marquardt, T., Stoykova, A., Spieler, D., Treichel, D., Ashery-Padan, R., Gruss, P., 2003. Retinal pigmented epithelium determination requires the redundant activities of Pax2 and Pax6. Development. 130, 2903-15.

Beche-Belsot, J. S., Planque, N., Martin, P., Saule, S., 2001. [The myc oncogene and transdifferentiation of the retinal pigment epithelium]. J Soc Biol. 195, 107-13.

Beebe, D. C., 1994. Homeobox genes and vertebrate eye development. Invest Ophthalmol Vis Sci. 35, 2897-900.

Bernardos, R. L., Barthel, L. K., Meyers, J. R., Raymond, P. A., 2007. Late-stage neuronal progenitors in the retina are radial Muller glia that function as retinal stem cells. J Neurosci. 27, 7028-40.

Bharti, K., Nguyen, M. T., Skuntz, S., Bertuzzi, S., Arnheiter, H., 2006. The other pigment cell: specification and development of the pigmented epithelium of the vertebrate eye. Pigment Cell Res. 19, 380-94.

Bopp, D., Burri, M., Baumgartner, S., Frigerio, G., Noll, M., 1986. Conservation of a large protein domain in the segmentation gene paired and in functionally related genes of Drosophila. Cell. 47, 1033-40.

Bora, N., Conway, S. J., Liang, H., Smith, S. B., 1998. Transient overexpression of the Microphthalmia gene in the eyes of Microphthalmia vitiligo mutant mice. Dev Dyn. 213, 283-92.

Bottcher, R. T., Niehrs, C., 2005. Fibroblast growth factor signaling during early vertebrate development. Endocr Rev. 26, 63-77.

Bovolenta, P., Mallamaci, A., Briata, P., Corte, G., Boncinelli, E., 1997. Implication of OTX2 in pigment epithelium determination and neural retina differentiation. J Neurosci. 17, 4243-52. 
Bovolenta, P., Mallamaci, A., Puelles, L., Boncinelli, E., 1998. Expression pattern of cSix3, a member of the Six/sine oculis family of transcription factors. Mech Dev. 70, 201-3.

Bumsted, K. M., Barnstable, C. J., 2000. Dorsal retinal pigment epithelium differentiates as neural retina in the microphthalmia (mi/mi) mouse. Invest Ophthalmol Vis Sci. 41, 903-8.

Burglin, T. R., A comprehensive classification of homeobox genes. In: D. Duboule, (Ed.), Guidebook to Homeobox Genes. Oxford City Press, New York, 1994, pp. 25-73.

Burmeister, M., Novak, J., Liang, M. Y., Basu, S., Ploder, L., Hawes, N. L., Vidgen, D., Hoover, F., Goldman, D., Kalnins, V. I., Roderick, T. H., Taylor, B. A., Hankin, M. H., McInnes, R. R., 1996. Ocular retardation mouse caused by Chx10 homeobox null allele: impaired retinal progenitor proliferation and bipolar cell differentiation. Nat Genet. 12, 376-84.

Carl, M., Loosli, F., Wittbrodt, J., 2002. Six3 inactivation reveals its essential role for the formation and patterning of the vertebrate eye. Development. 129, 4057-63.

Casarosa, S., Amato, M. A., Andreazzoli, M., Gestri, G., Barsacchi, G., Cremisi, F., 2003. Xrx1 controls proliferation and multipotency of retinal progenitors. Mol Cell Neurosci. 22, 25-36.

Casarosa, S., Andreazzoli, M., Simeone, A., Barsacchi, G., 1997. Xrx1, a novel Xenopus homeobox gene expressed during eye and pineal gland development. Mech Dev. 61, 187-98.

Chase, H. B., Chase, E. B., 1941. Studies of an anophthalmic strain of mice. I. Embryology of the eye region. J Morph. 68, 279-301.

Chow, R. L., Altmann, C. R., Lang, R. A., Hemmati-Brivanlou, A., 1999. Pax6 induces ectopic eyes in a vertebrate. Development. 126, 4213-22.

Chow, R. L., Lang, R. A., 2001. Early eye development in vertebrates. Annu Rev Cell Dev Biol. 17, 25596.

Chow, R. L., Roux, G. D., Roghani, M., Palmer, M. A., Rifkin, D. B., Moscatelli, D. A., Lang, R. A., 1995. FGF suppresses apoptosis and induces differentiation of fibre cells in the mouse lens. Development. 121, 4383-93.

Chuang, J. C., Mathers, P. H., Raymond, P. A., 1999. Expression of three Rx homeobox genes in embryonic and adult zebrafish. Mech Dev. 84, 195-8.

Collinson, J. M., Hill, R. E., West, J. D., 2000. Different roles for Pax6 in the optic vesicle and facial epithelium mediate early morphogenesis of the murine eye. Development. 127, 945-56.

Colvin, J. S., Feldman, B., Nadeau, J. H., Goldfarb, M., Ornitz, D. M., 1999. Genomic organization and embryonic expression of the mouse fibroblast growth factor 9 gene. Dev Dyn. 216, 72-88.

Corson, L. B., Yamanaka, Y., Lai, K. M., Rossant, J., 2003. Spatial and temporal patterns of ERK signaling during mouse embryogenesis. Development. 130, 4527-37.

Coulombre, J. L., Coulombre, A. J., 1965. Regeneration of neural retina from the pigmented epithelium in the chick embryo. Dev Biol. 12, 79-92.

Cunningham, J. J., Levine, E. M., Zindy, F., Goloubeva, O., Roussel, M. F., Smeyne, R. J., 2002. The cyclin-dependent kinase inhibitors p19(Ink4d) and p27(Kip1) are coexpressed in select retinal cells and act cooperatively to control cell cycle exit. Mol Cell Neurosci. 19, 359-74.

Danno, H., Michiue, T., Hitachi, K., Yukita, A., Ishiura, S., Asashima, M., 2008. Molecular links among the causative genes for ocular malformation: Otx2 and Sox 2 coregulate Rax expression. Proc Natl Acad Sci U S A. 105, 5408-13.

de Iongh, R., McAvoy, J. W., 1993. Spatio-temporal distribution of acidic and basic FGF indicates a role for FGF in rat lens morphogenesis. Dev Dyn. 198, 190-202.

Del Bene, F., Tessmar-Raible, K., Wittbrodt, J., 2004. Direct interaction of geminin and Six3 in eye development. Nature. 427, 745-9.

Deschet, K., Bourrat, F., Ristoratore, F., Chourrout, D., Joly, J. S., 1999. Expression of the medaka (Oryzias latipes) Ol-Rx3 paired-like gene in two diencephalic derivatives, the eye and the hypothalamus. Mech Dev. 83, 179-82.

Dudley, A. T., Lyons, K. M., Robertson, E. J., 1995. A requirement for bone morphogenetic protein-7 during development of the mammalian kidney and eye. Genes Dev. 9, 2795-807. 
Dyer, M. A., Cepko, C. L., 2001. p27Kip1 and p57Kip2 regulate proliferation in distinct retinal progenitor cell populations. J Neurosci. 21, 4259-71.

Dymecki, S. M., 1996. Flp recombinase promotes site-specific DNA recombination in embryonic stem cells and transgenic mice. Proc Natl Acad Sci U S A. 93, 6191-6.

Faber, S. C., Dimanlig, P., Makarenkova, H. P., Shirke, S., Ko, K., Lang, R. A., 2001. Fgf receptor signaling plays a role in lens induction. Development. 128, 4425-38.

Fantes, J., Ragge, N. K., Lynch, S. A., McGill, N. I., Collin, J. R., Howard-Peebles, P. N., Hayward, C., Vivian, A. J., Williamson, K., van Heyningen, V., FitzPatrick, D. R., 2003. Mutations in SOX2 cause anophthalmia. Nat Genet. 33, 461-3.

Ferda Percin, E., Ploder, L. A., Yu, J. J., Arici, K., Horsford, D. J., Rutherford, A., Bapat, B., Cox, D. W., Duncan, A. M., Kalnins, V. I., Kocak-Altintas, A., Sowden, J. C., Traboulsi, E., Sarfarazi, M., McInnes, R. R., 2000. Human microphthalmia associated with mutations in the retinal homeobox gene CHX10. Nat Genet. 25, 397-401.

Forman-Kay, J. D., Pawson, T., 1999. Diversity in protein recognition by PTB domains. Curr Opin Struct Biol. 9, 690-5.

Fuhrmann, S., Levine, E. M., Reh, T. A., 2000. Extraocular mesenchyme patterns the optic vesicle during early eye development in the embryonic chick. Development. 127, 4599-609.

Fujimura, N., Taketo, M. M., Mori, M., Korinek, V., Kozmik, Z., 2009. Spatial and temporal regulation of Wnt/beta-catenin signaling is essential for development of the retinal pigment epithelium. Dev Biol. 334, 31-45.

Furukawa, T., Kozak, C. A., Cepko, C. L., 1997. rax, a novel paired-type homeobox gene, shows expression in the anterior neural fold and developing retina. Proc Natl Acad Sci U S A. 94, 308893.

Furukawa, T., Mukherjee, S., Bao, Z. Z., Morrow, E. M., Cepko, C. L., 2000. rax, Hes1, and notch1 promote the formation of Muller glia by postnatal retinal progenitor cells. Neuron. 26, 383-94.

Furuta, Y., Hogan, B. L., 1998. BMP4 is essential for lens induction in the mouse embryo. Genes Dev. $12,3764-75$.

Furuta, Y., Lagutin, O., Hogan, B. L., Oliver, G. C., 2000. Retina- and ventral forebrain-specific Cre recombinase activity in transgenic mice. Genesis. 26, 130-2.

Galliot, B., de Vargas, C., Miller, D., 1999. Evolution of homeobox genes: Q50 Paired-like genes founded the Paired class. Dev Genes Evol. 209, 186-97.

Galy, A., Neron, B., Planque, N., Saule, S., Eychene, A., 2002. Activated MAPK/ERK kinase (MEK-1) induces transdifferentiation of pigmented epithelium into neural retina. Dev Biol. 248, 251-64.

Gestri, G., Carl, M., Appolloni, I., Wilson, S. W., Barsacchi, G., Andreazzoli, M., 2005. Six3 functions in anterior neural plate specification by promoting cell proliferation and inhibiting Bmp4 expression. Development. 132, 2401-13.

Glaser, T., Walton, D. S., Maas, R. L., 1992. Genomic structure, evolutionary conservation and aniridia mutations in the human PAX6 gene. Nat Genet. 2, 232-9.

Gotoh, N., Ito, M., Yamamoto, S., Yoshino, I., Song, N., Wang, Y., Lax, I., Schlessinger, J., Shibuya, M., Lang, R. A., 2004. Tyrosine phosphorylation sites on FRS2alpha responsible for Shp2 recruitment are critical for induction of lens and retina. Proc Natl Acad Sci U S A. 101, 17144-9.

Govindarajan, V., Overbeek, P. A., 2001. Secreted FGFR3, but not FGFR1, inhibits lens fiber differentiation. Development. 128, 1617-27.

Granadino, B., Gallardo, M. E., Lopez-Rios, J., Sanz, R., Ramos, C., Ayuso, C., Bovolenta, P., Rodriguez de Cordoba, S., 1999. Genomic cloning, structure, expression pattern, and chromosomal location of the human SIX3 gene. Genomics. 55, 100-5.

Green, E. S., Stubbs, J. L., Levine, E. M., 2003. Genetic rescue of cell number in a mouse model of microphthalmia: interactions between Chx10 and G1-phase cell cycle regulators. Development. $130,539-52$. 
Grindley, J. C., Davidson, D. R., Hill, R. E., 1995. The role of Pax-6 in eye and nasal development. Development. 121, 1433-42.

Gu, H., Zou, Y. R., Rajewsky, K., 1993. Independent control of immunoglobulin switch recombination at individual switch regions evidenced through Cre-loxP-mediated gene targeting. Cell. 73, $1155-$ 64.

Guillemot, F., Cepko, C. L., 1992. Retinal fate and ganglion cell differentiation are potentiated by acidic FGF in an in vitro assay of early retinal development. Development. 114, 743-54.

Hagstrom, S. A., Pauer, G. J., Reid, J., Simpson, E., Crowe, S., Maumenee, I. H., Traboulsi, E. I., 2005. SOX2 mutation causes anophthalmia, hearing loss, and brain anomalies. Am J Med Genet A. 138A, 95-8.

Halder, G., Callaerts, P., Gehring, W. J., 1995. Induction of ectopic eyes by targeted expression of the eyeless gene in Drosophila. Science. 267, 1788-92.

Hanson, I. M., Fletcher, J. M., Jordan, T., Brown, A., Taylor, D., Adams, R. J., Punnett, H. H., van Heyningen, V., 1994. Mutations at the PAX6 locus are found in heterogeneous anterior segment malformations including Peters' anomaly. Nat Genet. 6, 168-73.

Hanson, I. M., Seawright, A., Hardman, K., Hodgson, S., Zaletayev, D., Fekete, G., van Heyningen, V., 1993. PAX6 mutations in aniridia. Hum Mol Genet. 2, 915-20.

Hebert, J. M., McConnell, S. K., 2000. Targeting of cre to the Foxg1 (BF-1) locus mediates loxP recombination in the telencephalon and other developing head structures. Dev Biol. 222, 296-306.

Herzog, W., Sonntag, C., von der Hardt, S., Roehl, H. H., Varga, Z. M., Hammerschmidt, M., 2004. Fgf3 signaling from the ventral diencephalon is required for early specification and subsequent survival of the zebrafish adenohypophysis. Development. 131, 3681-92.

Hill, R. E., Favor, J., Hogan, B. L., Ton, C. C., Saunders, G. F., Hanson, I. M., Prosser, J., Jordan, T., Hastie, N. D., van Heyningen, V., 1991. Mouse small eye results from mutations in a paired-like homeobox-containing gene. Nature. 354, 522-5.

Hirsch, N., Zimmerman, L. B., Gray, J., Chae, J., Curran, K. L., Fisher, M., Ogino, H., Grainger, R. M., 2002. Xenopus tropicalis transgenic lines and their use in the study of embryonic induction. Dev Dyn. 225, 522-35.

Hodgkinson, C. A., Moore, K. J., Nakayama, A., Steingrimsson, E., Copeland, N. G., Jenkins, N. A., Arnheiter, H., 1993. Mutations at the mouse microphthalmia locus are associated with defects in a gene encoding a novel basic-helix-loop-helix-zipper protein. Cell. 74, 395-404.

Hoess, R. H., Abremski, K., 1985. Mechanism of strand cleavage and exchange in the Cre-lox sitespecific recombination system. J Mol Biol. 181, 351-62.

Hogan, B. L., Horsburgh, G., Cohen, J., Hetherington, C. M., Fisher, G., Lyon, M. F., 1986. Small eyes (Sey): a homozygous lethal mutation on chromosome 2 which affects the differentiation of both lens and nasal placodes in the mouse. J Embryol Exp Morphol. 97, 95-110.

Holtfreter, J., 1939. Gewebeaffinitat, ein Mittel der embryonalen Formbildung. Arch Exp Zellforsch. 23, 169-209.

Holzenberger, M., Lenzner, C., Leneuve, P., Zaoui, R., Hamard, G., Vaulont, S., Bouc, Y. L., 2000. Cremediated germline mosaicism: a method allowing rapid generation of several alleles of a target gene. Nucleic Acids Res. 28, E92.

Horsford, D. J., Nguyen, M. T., Sellar, G. C., Kothary, R., Arnheiter, H., McInnes, R. R., 2005. Chx10 repression of Mitf is required for the maintenance of mammalian neuroretinal identity. Development. 132, 177-87.

Hyer, J., Kuhlman, J., Afif, E., Mikawa, T., 2003. Optic cup morphogenesis requires pre-lens ectoderm but not lens differentiation. Dev Biol. 259, 351-63.

Hyer, J., Mima, T., Mikawa, T., 1998. FGF1 patterns the optic vesicle by directing the placement of the neural retina domain. Development. 125, 869-77.

Ikeda, Y., 1937. Uber die Bildug Akzessorischer Retina aus dem Tapetum bei Hybnobius. Arch fur Entw -mech. 136, 676-80. 
Itoh, N., Ornitz, D. M., 2004. Evolution of the Fgf and Fgfr gene families. Trends Genet. 20, 563-9.

Jackson, I. J., Chambers, D. M., Budd, P. S., Johnson, R., 1991. The tyrosinase-related protein-1 gene has a structure and promoter sequence very different from tyrosinase. Nucleic Acids Res. 19, 3799804.

Johnson, D. E., Lee, P. L., Lu, J., Williams, L. T., 1990. Diverse forms of a receptor for acidic and basic fibroblast growth factors. Mol Cell Biol. 10, 4728-36.

Jordan, T., Hanson, I., Zaletayev, D., Hodgson, S., Prosser, J., Seawright, A., Hastie, N., van Heyningen, V., 1992. The human PAX6 gene is mutated in two patients with aniridia. Nat Genet. 1, 328-32.

Kamachi, Y., Sockanathan, S., Liu, Q., Breitman, M., Lovell-Badge, R., Kondoh, H., 1995. Involvement of SOX proteins in lens-specific activation of crystallin genes. EMBO J. 14, 3510-9.

Kamachi, Y., Uchikawa, M., Collignon, J., Lovell-Badge, R., Kondoh, H., 1998. Involvement of Sox1, 2 and 3 in the early and subsequent molecular events of lens induction. Development. 125, 252132.

Kamachi, Y., Uchikawa, M., Tanouchi, A., Sekido, R., Kondoh, H., 2001. Pax6 and SOX2 form a coDNA-binding partner complex that regulates initiation of lens development. Genes Dev. 15, 1272-86.

Kammandel, B., Chowdhury, K., Stoykova, A., Aparicio, S., Brenner, S., Gruss, P., 1999. Distinct cisessential modules direct the time-space pattern of the Pax6 gene activity. Dev Biol. 205, 79-97.

Kawakami, K., Ohto, H., Takizawa, T., Saito, T., 1996. Identification and expression of six family genes in mouse retina. FEBS Lett. 393, 259-63.

Kennedy, B. N., Stearns, G. W., Smyth, V. A., Ramamurthy, V., van Eeden, F., Ankoudinova, I., Raible, D., Hurley, J. B., Brockerhoff, S. E., 2004. Zebrafish rx3 and mab2112 are required during eye morphogenesis. Dev Biol. 270, 336-49.

Kimura, A., Singh, D., Wawrousek, E. F., Kikuchi, M., Nakamura, M., Shinohara, T., 2000. Both PCE1/RX and OTX/CRX interactions are necessary for photoreceptor-specific gene expression. J Biol Chem. 275, 1152-60.

Klint, P., Claesson-Welsh, L., 1999. Signal transduction by fibroblast growth factor receptors. Front Biosci. 4, D165-77.

Kobayashi, M., Nishikawa, K., Suzuki, T., Yamamoto, M., 2001. The homeobox protein Six3 interacts with the Groucho corepressor and acts as a transcriptional repressor in eye and forebrain formation. Dev Biol. 232, 315-26.

Kouhara, H., Hadari, Y. R., Spivak-Kroizman, T., Schilling, J., Bar-Sagi, D., Lax, I., Schlessinger, J., 1997. A lipid-anchored Grb2-binding protein that links FGF-receptor activation to the Ras/MAPK signaling pathway. Cell. 89, 693-702.

Lagutin, O., Zhu, C. C., Furuta, Y., Rowitch, D. H., McMahon, A. P., Oliver, G., 2001. Six3 promotes the formation of ectopic optic vesicle-like structures in mouse embryos. Dev Dyn. 221, 342-9.

Lagutin, O. V., Zhu, C. C., Kobayashi, D., Topczewski, J., Shimamura, K., Puelles, L., Russell, H. R., McKinnon, P. J., Solnica-Krezel, L., Oliver, G., 2003. Six3 repression of Wnt signaling in the anterior neuroectoderm is essential for vertebrate forebrain development. Genes Dev. 17, 368-79.

Lang, R. A., 2004. Pathways regulating lens induction in the mouse. Int J Dev Biol. 48, 783-91.

Lanning, J. L., Wallace, J. S., Zhang, D., Diwakar, G., Jiao, Z., Hornyak, T. J., 2005. Altered melanocyte differentiation and retinal pigmented epithelium transdifferentiation induced by Mash1 expression in pigment cell precursors. J Invest Dermatol. 125, 805-17.

Le, R. D., Rayner, K., Rex, M., Wigmore, P. M., Scotting, P. J., 2002. The transcription factor cSox 2 and Neuropeptide Y define a novel subgroup of amacrine cells in the retina. J Anat. 200, 51-6.

Lee, C. S., May, N. R., Fan, C. M., 2001. Transdifferentiation of the ventral retinal pigmented epithelium to neural retina in the growth arrest specific gene 1 mutant. Dev Biol. 236, 17-29.

Lee, P. L., Johnson, D. E., Cousens, L. S., Fried, V. A., Williams, L. T., 1989. Purification and complementary DNA cloning of a receptor for basic fibroblast growth factor. Science. 245, 5760. 
Lequeux, L., Rio, M., Vigouroux, A., Titeux, M., Etchevers, H., Malecaze, F., Chassaing, N., Calvas, P., 2008. Confirmation of RAX gene involvement in human anophthalmia. Clin Genet. 74, 392-5.

Levine, E. M., Close, J., Fero, M., Ostrovsky, A., Reh, T. A., 2000. p27(Kip1) regulates cell cycle withdrawal of late multipotent progenitor cells in the mammalian retina. Dev Biol. 219, 299-314.

Li, H., Tierney, C., Wen, L., Wu, J. Y., Rao, Y., 1997. A single morphogenetic field gives rise to two retina primordia under the influence of the prechordal plate. Development. 124, 603-15.

Lin, Y. P., Ouchi, Y., Satoh, S., Watanabe, S., 2009. Sox2 plays a role in the induction of amacrine and Muller glial cells in mouse retinal progenitor cells. Invest Ophthalmol Vis Sci. 50, 68-74.

Liu, I. S., Chen, J. D., Ploder, L., Vidgen, D., van der Kooy, D., Kalnins, V. I., McInnes, R. R., 1994. Developmental expression of a novel murine homeobox gene (Chx10): evidence for roles in determination of the neuroretina and inner nuclear layer. Neuron. 13, 377-93.

London, N. J., Kessler, P., Williams, B., Pauer, G. J., Hagstrom, S. A., Traboulsi, E. I., 2009. Sequence alterations in RX in patients with microphthalmia, anophthalmia, and coloboma. Mol Vis. 15, $162-7$.

Loosli, F., Koster, R. W., Carl, M., Krone, A., Wittbrodt, J., 1998. Six3, a medaka homologue of the Drosophila homeobox gene sine oculis is expressed in the anterior embryonic shield and the developing eye. Mech Dev. 74, 159-64.

Loosli, F., Staub, W., Finger-Baier, K. C., Ober, E. A., Verkade, H., Wittbrodt, J., Baier, H., 2003. Loss of eyes in zebrafish caused by mutation of chokh/rx3. EMBO Rep. 4, 894-9.

Loosli, F., Winkler, S., Burgtorf, C., Wurmbach, E., Ansorge, W., Henrich, T., Grabher, C., Arendt, D., Carl, M., Krone, A., Grzebisz, E., Wittbrodt, J., 2001. Medaka eyeless is the key factor linking retinal determination and eye growth. Development. 128, 4035-44.

Loosli, F., Winkler, S., Wittbrodt, J., 1999. Six3 overexpression initiates the formation of ectopic retina. Genes Dev. 13, 649-54.

Lopashov, G. V., Stroeva, O. G., 1964. Development of the Eye. Davey \& Co., New York.

Lopez-Rios, J., Tessmar, K., Loosli, F., Wittbrodt, J., Bovolenta, P., 2003. Six3 and Six6 activity is modulated by members of the groucho family. Development. 130, 185-95.

Lord-Grignon, J., Abdouh, M., Bernier, G., 2006. Identification of genes expressed in retinal progenitor/stem cell colonies isolated from the ocular ciliary body of adult mice. Gene Expr Patterns. 6, 992-9.

Lovicu, F. J., McAvoy, J. W., 2005. Growth factor regulation of lens development. Dev Biol. 280, 1-14.

Lovicu, F. J., Overbeek, P. A., 1998. Overlapping effects of different members of the FGF family on lens fiber differentiation in transgenic mice. Development. 125, 3365-77.

Ma, W., Yan, R. T., Li, X., Wang, S. Z., 2009. Reprogramming retinal pigment epithelium to differentiate toward retinal neurons with Sox2. Stem Cells. 27, 1376-87.

Mao, X., Fujiwara, Y., Chapdelaine, A., Yang, H., Orkin, S. H., 2001. Activation of EGFP expression by Cre-mediated excision in a new ROSA26 reporter mouse strain. Blood. 97, 324-6.

Marquardt, T., Ashery-Padan, R., Andrejewski, N., Scardigli, R., Guillemot, F., Gruss, P., 2001. Pax6 is required for the multipotent state of retinal progenitor cells. Cell. 105, 43-55.

Marquardt, T., Gruss, P., 2002. Generating neuronal diversity in the retina: one for nearly all. Trends Neurosci. 25, 32-8.

Martinelli, D. C., Fan, C. M., 2007. The role of Gas1 in embryonic development and its implications for human disease. Cell Cycle. 6, 2650-5.

Martinez-Morales, J. R., Del Bene, F., Nica, G., Hammerschmidt, M., Bovolenta, P., Wittbrodt, J., 2005. Differentiation of the vertebrate retina is coordinated by an FGF signaling center. Dev Cell. 8, 565-74.

Martinez-Morales, J. R., Dolez, V., Rodrigo, I., Zaccarini, R., Leconte, L., Bovolenta, P., Saule, S., 2003. OTX2 activates the molecular network underlying retina pigment epithelium differentiation. $\mathrm{J}$ Biol Chem. 278, 21721-31. 
Mathers, P. H., Grinberg, A., Mahon, K. A., Jamrich, M., 1997. The Rx homeobox gene is essential for vertebrate eye development. Nature. 387, 603-7.

Matsuda, T., Cepko, C. L., 2007. Controlled expression of transgenes introduced by in vivo electroporation. Proc Natl Acad Sci U S A. 104, 1027-32.

Matsuo, I., Kuratani, S., Kimura, C., Takeda, N., Aizawa, S., 1995. Mouse Otx2 functions in the formation and patterning of rostral head. Genes Dev. 9, 2646-58.

Matsuo, T., Osumi-Yamashita, N., Noji, S., Ohuchi, H., Koyama, E., Myokai, F., Matsuo, N., Taniguchi, S., Doi, H., Iseki, S., et al., 1993. A mutation in the Pax-6 gene in rat small eye is associated with impaired migration of midbrain crest cells. Nat Genet. 3, 299-304.

McAvoy, J. W., Chamberlain, C. G., 1989. Fibroblast growth factor (FGF) induces different responses in lens epithelial cells depending on its concentration. Development. 107, 221-8.

McWhirter, J. R., Goulding, M., Weiner, J. A., Chun, J., Murre, C., 1997. A novel fibroblast growth factor gene expressed in the developing nervous system is a downstream target of the chimeric homeodomain oncoprotein E2A-Pbx1. Development. 124, 3221-32.

Medina-Martinez, O., Amaya-Manzanares, F., Liu, C., Mendoza, M., Shah, R., Zhang, L., Behringer, R. R., Mahon, K. A., Jamrich, M., 2009. Cell-autonomous requirement for rx function in the mammalian retina and posterior pituitary. PLoS One. 4, e4513.

Mikami, Y., 1939. Reciprocal transformation of the parts in the developing eye-vesicle, with special reference to the inductive influence of the lens-ectoderm on the retinal determination. Zool Mag. 51, 253-56.

Miki, T., Bottaro, D. P., Fleming, T. P., Smith, C. L., Burgess, W. H., Chan, A. M., Aaronson, S. A., 1992. Determination of ligand-binding specificity by alternative splicing: two distinct growth factor receptors encoded by a single gene. Proc Natl Acad Sci U S A. 89, 246-50.

Mikkola, I., Bruun, J. A., Bjorkoy, G., Holm, T., Johansen, T., 1999. Phosphorylation of the transactivation domain of Pax6 by extracellular signal-regulated kinase and p38 mitogenactivated protein kinase. J Biol Chem. 274, 15115-26.

Mikkola, I., Bruun, J. A., Holm, T., Johansen, T., 2001. Superactivation of Pax6-mediated transactivation from paired domain-binding sites by dna-independent recruitment of different homeodomain proteins. J Biol Chem. 276, 4109-18.

Miller, D. L., Ortega, S., Bashayan, O., Basch, R., Basilico, C., 2000. Compensation by fibroblast growth factor 1 (FGF1) does not account for the mild phenotypic defects observed in FGF2 null mice. Mol Cell Biol. 20, 2260-8.

Mochii, M., Mazaki, Y., Mizuno, N., Hayashi, H., Eguchi, G., 1998a. Role of Mitf in differentiation and transdifferentiation of chicken pigmented epithelial cell. Dev Biol. 193, 47-62.

Mochii, M., Ono, T., Matsubara, Y., Eguchi, G., 1998b. Spontaneous transdifferentiation of quail pigmented epithelial cell is accompanied by a mutation in the Mitf gene. Dev Biol. 196, 145-59.

Mohammadi, M., Olsen, S. K., Ibrahimi, O. A., 2005. Structural basis for fibroblast growth factor receptor activation. Cytokine Growth Factor Rev. 16, 107-37.

Muller, F., Rohrer, H., Vogel-Hopker, A., 2007. Bone morphogenetic proteins specify the retinal pigment epithelium in the chick embryo. Development. 134, 3483-93.

Nakayama, A., Nguyen, M. T., Chen, C. C., Opdecamp, K., Hodgkinson, C. A., Arnheiter, H., 1998. Mutations in microphthalmia, the mouse homolog of the human deafness gene MITF, affect neuroepithelial and neural crest-derived melanocytes differently. Mech Dev. 70, 155-66.

Nakayama, K., Ishida, N., Shirane, M., Inomata, A., Inoue, T., Shishido, N., Horii, I., Loh, D. Y., 1996. Mice lacking p27(Kip1) display increased body size, multiple organ hyperplasia, retinal dysplasia, and pituitary tumors. Cell. 85, 707-20.

Nguyen, M., Arnheiter, H., 2000. Signaling and transcriptional regulation in early mammalian eye development: a link between FGF and MITF. Development. 127, 3581-91. 
Nishida, A., Furukawa, A., Koike, C., Tano, Y., Aizawa, S., Matsuo, I., Furukawa, T., 2003. Otx2 homeobox gene controls retinal photoreceptor cell fate and pineal gland development. Nat Neurosci. 6, 1255-63.

O'Gorman, S., Fox, D. T., Wahl, G. M., 1991. Recombinase-mediated gene activation and site-specific integration in mammalian cells. Science. 251, 1351-5.

Oliver, G., Loosli, F., Koster, R., Wittbrodt, J., Gruss, P., 1996. Ectopic lens induction in fish in response to the murine homeobox gene Six3. Mech Dev. 60, 233-9.

Oliver, G., Mailhos, A., Wehr, R., Copeland, N. G., Jenkins, N. A., Gruss, P., 1995. Six3, a murine homologue of the sine oculis gene, demarcates the most anterior border of the developing neural plate and is expressed during eye development. Development. 121, 4045-55.

Opas, M., Dziak, E., 1994. bFGF-induced transdifferentiation of RPE to neuronal progenitors is regulated by the mechanical properties of the substratum. Dev Biol. 161, 440-54.

Ornitz, D. M., Itoh, N., 2001. Fibroblast growth factors. Genome Biol. 2, REVIEWS3005.

Orr-Urtreger, A., Bedford, M. T., Burakova, T., Arman, E., Zimmer, Y., Yayon, A., Givol, D., Lonai, P., 1993. Developmental localization of the splicing alternatives of fibroblast growth factor receptor2 (FGFR2). Dev Biol. 158, 475-86.

Packer, S. O., 1967. The eye and skeletal effects of two mutant alleles at the microphthalmia locus of Mus musculus. J Exp Zool. 165, 21-45.

Pannese, M., Polo, C., Andreazzoli, M., Vignali, R., Kablar, B., Barsacchi, G., Boncinelli, E., 1995. The Xenopus homologue of Otx2 is a maternal homeobox gene that demarcates and specifies anterior body regions. Development. 121, 707-20.

Park, C. M., Hollenberg, M. J., 1989. Basic fibroblast growth factor induces retinal regeneration in vivo. Dev Biol. 134, 201-5.

Park, C. M., Hollenberg, M. J., 1991. Induction of retinal regeneration in vivo by growth factors. Dev Biol. 148, 322-33.

Park, I. H., Zhao, R., West, J. A., Yabuuchi, A., Huo, H., Ince, T. A., Lerou, P. H., Lensch, M. W., Daley, G. Q., 2008. Reprogramming of human somatic cells to pluripotency with defined factors. Nature. 451, 141-6.

Pawson, T., Olivier, P., Rozakis-Adcock, M., McGlade, J., Henkemeyer, M., 1993. Proteins with SH2 and SH3 domains couple receptor tyrosine kinases to intracellular signalling pathways. Philos Trans R Soc Lond B Biol Sci. 340, 279-85.

Pearson, B. J., Doe, C. Q., 2004. Specification of temporal identity in the developing nervous system. Annu Rev Cell Dev Biol. 20, 619-47.

Pevny, L., Placzek, M., 2005. SOX genes and neural progenitor identity. Curr Opin Neurobiol. 15, 7-13.

Pittack, C., Grunwald, G. B., Reh, T. A., 1997. Fibroblast growth factors are necessary for neural retina but not pigmented epithelium differentiation in chick embryos. Development. 124, 805-16.

Pittack, C., Jones, M., Reh, T. A., 1991. Basic fibroblast growth factor induces retinal pigment epithelium to generate neural retina in vitro. Development. 113, 577-88.

Planque, N., Leconte, L., Coquelle, F. M., Martin, P., Saule, S., 2001. Specific Pax-6/microphthalmia transcription factor interactions involve their DNA-binding domains and inhibit transcriptional properties of both proteins. J Biol Chem. 276, 29330-7.

Planque, N., Turque, N., Opdecamp, K., Bailly, M., Martin, P., Saule, S., 1999. Expression of the microphthalmia-associated basic helix-loop-helix leucine zipper transcription factor Mi in avian neuroretina cells induces a pigmented phenotype. Cell Growth Differ. 10, 525-36.

Plotnikov, A. N., Hubbard, S. R., Schlessinger, J., Mohammadi, M., 2000. Crystal structures of two FGFFGFR complexes reveal the determinants of ligand-receptor specificity. Cell. 101, 413-24.

Prigent, C., Dimitrov, S., 2003. Phosphorylation of serine 10 in histone H3, what for? J Cell Sci. 116, 3677-85.

Quinn, J. C., West, J. D., Hill, R. E., 1996. Multiple functions for Pax6 in mouse eye and nasal development. Genes Dev. 10, 435-46. 
Ragge, N. K., Lorenz, B., Schneider, A., Bushby, K., de Sanctis, L., de Sanctis, U., Salt, A., Collin, J. R., Vivian, A. J., Free, S. L., Thompson, P., Williamson, K. A., Sisodiya, S. M., van Heyningen, V., Fitzpatrick, D. R., 2005. SOX2 anophthalmia syndrome. Am J Med Genet A. 135, 1-7; discussion 8.

Rapraeger, A. C., Krufka, A., Olwin, B. B., 1991. Requirement of heparan sulfate for bFGF-mediated fibroblast growth and myoblast differentiation. Science. 252, 1705-8.

Rasmussen, J. T., Deardorff, M. A., Tan, C., Rao, M. S., Klein, P. S., Vetter, M. L., 2001. Regulation of eye development by frizzled signaling in Xenopus. Proc Natl Acad Sci U S A. 98, 3861-6.

Reh, T. A., Jones, M., Pittack, C., 1991. Common mechanisms of retinal regeneration in the larval frog and embryonic chick. Ciba Found Symp. 160, 192-204; discussion 204-8.

Rembold, M., Loosli, F., Adams, R. J., Wittbrodt, J., 2006. Individual cell migration serves as the driving force for optic vesicle evagination. Science. 313, 1130-4.

Reuss, B., von Bohlen und Halbach, O., 2003. Fibroblast growth factors and their receptors in the central nervous system. Cell Tissue Res. 313, 139-57.

Robinson, M. L., 2006. An essential role for FGF receptor signaling in lens development. Semin Cell Dev Biol. 17, 726-40.

Robinson, M. L., MacMillan-Crow, L. A., Thompson, J. A., Overbeek, P. A., 1995. Expression of a truncated FGF receptor results in defective lens development in transgenic mice. Development. 121, 3959-67.

Rojas-Munoz, A., Dahm, R., Nusslein-Volhard, C., 2005. chokh/rx3 specifies the retinal pigment epithelium fate independently of eye morphogenesis. Dev Biol. 288, 348-62.

Rousseau, B., Larrieu-Lahargue, F., Bikfalvi, A., Javerzat, S., 2003. Involvement of fibroblast growth factors in choroidal angiogenesis and retinal vascularization. Exp Eye Res. 77, 147-56.

Rowan, S., Chen, C. M., Young, T. L., Fisher, D. E., Cepko, C. L., 2004. Transdifferentiation of the retina into pigmented cells in ocular retardation mice defines a new function of the homeodomain gene Chx10. Development. 131, 5139-52.

Russell, C., 2003. The roles of Hedgehogs and Fibroblast Growth Factors in eye development and retinal cell rescue. Vision Res. 43, 899-912.

Sakaguchi, D. S., Janick, L. M., Reh, T. A., 1997. Basic fibroblast growth factor (FGF-2) induced transdifferentiation of retinal pigment epithelium: generation of retinal neurons and glia. Dev Dyn. 209, 387-98.

Sakami, S., Etter, P., Reh, T. A., 2008. Activin signaling limits the competence for retinal regeneration from the pigmented epithelium. Mech Dev. 125, 106-16.

Sauer, B., Henderson, N., 1988. Site-specific DNA recombination in mammalian cells by the Cre recombinase of bacteriophage P1. Proc Natl Acad Sci U S A. 85, 5166-70.

Schedl, A., Ross, A., Lee, M., Engelkamp, D., Rashbass, P., van Heyningen, V., Hastie, N. D., 1996. Influence of PAX6 gene dosage on development: overexpression causes severe eye abnormalities. Cell. 86, 71-82.

Schlessinger, J., 2000. Cell signaling by receptor tyrosine kinases. Cell. 103, 211-25.

Scholtz, C. L., Chan, K. K., 1987. Complicated colobomatous microphthalmia in the microphthalmic (mi/mi) mouse. Development. 99, 501-8.

Schwarz, M., Cecconi, F., Bernier, G., Andrejewski, N., Kammandel, B., Wagner, M., Gruss, P., 2000. Spatial specification of mammalian eye territories by reciprocal transcriptional repression of Pax2 and Pax6. Development. 127, 4325-34.

Shanmugalingam, S., Houart, C., Picker, A., Reifers, F., Macdonald, R., Barth, A., Griffin, K., Brand, M., Wilson, S. W., 2000. Ace/Fgf8 is required for forebrain commissure formation and patterning of the telencephalon. Development. 127, 2549-61.

Shibahara, S., Takeda, K., Yasumoto, K., Udono, T., Watanabe, K., Saito, H., Takahashi, K., 2001. Microphthalmia-associated transcription factor (MITF): multiplicity in structure, function, and regulation. J Investig Dermatol Symp Proc. 6, 99-104. 
Shibata, H., Toyama, K., Shioya, H., Ito, M., Hirota, M., Hasegawa, S., Matsumoto, H., Takano, H., Akiyama, T., Toyoshima, K., Kanamaru, R., Kanegae, Y., Saito, I., Nakamura, Y., Shiba, K., Noda, T., 1997. Rapid colorectal adenoma formation initiated by conditional targeting of the Apc gene. Science. 278, 120-3.

Sicinski, P., Donaher, J. L., Parker, S. B., Li, T., Fazeli, A., Gardner, H., Haslam, S. Z., Bronson, R. T., Elledge, S. J., Weinberg, R. A., 1995. Cyclin D1 provides a link between development and oncogenesis in the retina and breast. Cell. 82, 621-30.

Silver, J., Hughes, A. F., 1974. The relationship between morphogenetic cell death and the development of congenital anophthalmia. J Comp Neurol. 157, 281-302.

Simeone, A., Acampora, D., Gulisano, M., Stornaiuolo, A., Boncinelli, E., 1992. Nested expression domains of four homeobox genes in developing rostral brain. Nature. 358, 687-90.

Simeone, A., Acampora, D., Mallamaci, A., Stornaiuolo, A., D'Apice, M. R., Nigro, V., Boncinelli, E., 1993. A vertebrate gene related to orthodenticle contains a homeodomain of the bicoid class and demarcates anterior neuroectoderm in the gastrulating mouse embryo. EMBO J. 12, 2735-47.

Simeone, A., Puelles, E., Acampora, D., 2002. The Otx family. Curr Opin Genet Dev. 12, 409-15.

Smith, S. B., Zhou, B. K., Orlow, S. J., 1998. Expression of tyrosinase and the tyrosinase related proteins in the Mitfvit (vitiligo) mouse eye: implications for the function of the microphthalmia transcription factor. Exp Eye Res. 66, 403-10.

Smith, S. T., Jaynes, J. B., 1996. A conserved region of engrailed, shared among all en-, gsc-, Nk1-, Nk2and msh-class homeoproteins, mediates active transcriptional repression in vivo. Development. 122, 3141-50.

Solomon, B. D., Lacbawan, F., Jain, M., Domene, S., Roessler, E., Moore, C., Dobyns, W. B., Muenke, M., 2009. A novel SIX3 mutation segregates with holoprosencephaly in a large family. Am J Med Genet A. 149A, 919-25.

Spemann, H., 1901. Uber Correlationen in der Entwickelung des Auges. Ver Anat Ges. 15, 61-79.

Spence, J. R., Madhavan, M., Aycinena, J. C., Del Rio-Tsonis, K., 2007. Retina regeneration in the chick embryo is not induced by spontaneous Mitf downregulation but requires FGF/FGFR/MEK/Erk dependent upregulation of Pax6. Mol Vis. 13, 57-65.

Spence, J. R., Madhavan, M., Ewing, J. D., Jones, D. K., Lehman, B. M., Del Rio-Tsonis, K., 2004. The hedgehog pathway is a modulator of retina regeneration. Development. 131, 4607-21.

Stauber, D. J., DiGabriele, A. D., Hendrickson, W. A., 2000. Structural interactions of fibroblast growth factor receptor with its ligands. Proc Natl Acad Sci U S A. 97, 49-54.

Steel, K. P., Davidson, D. R., Jackson, I. J., 1992. TRP-2/DT, a new early melanoblast marker, shows that steel growth factor (c-kit ligand) is a survival factor. Development. 115, 1111-9.

Sternberg, N., Hamilton, D., 1981. Bacteriophage P1 site-specific recombination. I. Recombination between loxP sites. J Mol Biol. 150, 467-86.

Sturm, R. A., Das, G., Herr, W., 1988. The ubiquitous octamer-binding protein Oct-1 contains a POU domain with a homeo box subdomain. Genes Dev. 2, 1582-99.

Tabata, Y., Ouchi, Y., Kamiya, H., Manabe, T., Arai, K., Watanabe, S., 2004. Specification of the retinal fate of mouse embryonic stem cells by ectopic expression of Rx/rax, a homeobox gene. Mol Cell Biol. 24, 4513-21.

Takahashi, K., Tanabe, K., Ohnuki, M., Narita, M., Ichisaka, T., Tomoda, K., Yamanaka, S., 2007. Induction of pluripotent stem cells from adult human fibroblasts by defined factors. Cell. 131, 861-72.

Takahashi, K., Yamanaka, S., 2006. Induction of pluripotent stem cells from mouse embryonic and adult fibroblast cultures by defined factors. Cell. 126, 663-76.

Taranova, O. V., Magness, S. T., Fagan, B. M., Wu, Y., Surzenko, N., Hutton, S. R., Pevny, L. H., 2006. SOX2 is a dose-dependent regulator of retinal neural progenitor competence. Genes Dev. 20, 1187-202. 
Tcheng, M., Fuhrmann, G., Hartmann, M. P., Courtois, Y., Jeanny, J. C., 1994. Spatial and temporal expression patterns of FGF receptor genes type 1 and type 2 in the developing chick retina. Exp Eye Res. 58, 351-8.

Terada, K., Kitayama, A., Kanamoto, T., Ueno, N., Furukawa, T., 2006. Nucleosome regulator Xhmgb3 is required for cell proliferation of the eye and brain as a downstream target of Xenopus rax/Rx1. Dev Biol. 291, 398-412.

Thisse, B., Thisse, C., 2005. Functions and regulations of fibroblast growth factor signaling during embryonic development. Dev Biol. 287, 390-402.

Tomita, K., Ishibashi, M., Nakahara, K., Ang, S. L., Nakanishi, S., Guillemot, F., Kageyama, R., 1996. Mammalian hairy and Enhancer of split homolog 1 regulates differentiation of retinal neurons and is essential for eye morphogenesis. Neuron. 16, 723-34.

Ton, C. C., Hirvonen, H., Miwa, H., Weil, M. M., Monaghan, P., Jordan, T., van Heyningen, V., Hastie, N. D., Meijers-Heijboer, H., Drechsler, M., et al., 1991. Positional cloning and characterization of a paired box- and homeobox-containing gene from the aniridia region. Cell. 67, 1059-74.

Torres, M., Gomez-Pardo, E., Gruss, P., 1996. Pax2 contributes to inner ear patterning and optic nerve trajectory. Development. 122, 3381-91.

Toy, J., Yang, J. M., Leppert, G. S., Sundin, O. H., 1998. The optx2 homeobox gene is expressed in early precursors of the eye and activates retina-specific genes. Proc Natl Acad Sci U S A. 95, 10643-8.

Truslove, G. M., 1962. A gene causing ocular retardation in the mouse. J Embryol Exp Morphol. 10, 65260.

Tsonis, P. A., Del Rio-Tsonis, K., 2004. Lens and retina regeneration: transdifferentiation, stem cells and clinical applications. Exp Eye Res. 78, 161-72.

Tucker, P., Laemle, L., Munson, A., Kanekar, S., Oliver, E. R., Brown, N., Schlecht, H., Vetter, M., Glaser, T., 2001. The eyeless mouse mutation (ey1) removes an alternative start codon from the Rx/rax homeobox gene. Genesis. 31, 43-53.

Uchikawa, M., Kamachi, Y., Kondoh, H., 1999. Two distinct subgroups of Group B Sox genes for transcriptional activators and repressors: their expression during embryonic organogenesis of the chicken. Mech Dev. 84, 103-20.

Uwanogho, D., Rex, M., Cartwright, E. J., Pearl, G., Healy, C., Scotting, P. J., Sharpe, P. T., 1995. Embryonic expression of the chicken Sox2, Sox3 and Sox 11 genes suggests an interactive role in neuronal development. Mech Dev. 49, 23-36.

Van Raay, T. J., Moore, K. B., Iordanova, I., Steele, M., Jamrich, M., Harris, W. A., Vetter, M. L., 2005. Frizzled 5 signaling governs the neural potential of progenitors in the developing Xenopus retina. Neuron. 46, 23-36.

Vogel-Hopker, A., Momose, T., Rohrer, H., Yasuda, K., Ishihara, L., Rapaport, D. H., 2000. Multiple functions of fibroblast growth factor-8 (FGF-8) in chick eye development. Mech Dev. 94, 25-36.

Vooijs, M., van der Valk, M., te Riele, H., Berns, A., 1998. Flp-mediated tissue-specific inactivation of the retinoblastoma tumor suppressor gene in the mouse. Oncogene. 17, 1-12.

Voronina, V. A., Kozhemyakina, E. A., O'Kernick, C. M., Kahn, N. D., Wenger, S. L., Linberg, J. V., Schneider, A. S., Mathers, P. H., 2004. Mutations in the human RAX homeobox gene in a patient with anophthalmia and sclerocornea. Hum Mol Genet. 13, 315-22.

Voronina, V. A., Kozlov, S., Mathers, P. H., Lewandoski, M., 2005. Conditional alleles for activation and inactivation of the mouse Rx homeobox gene. Genesis. 41, 160-4.

Voronina, V. A., Kozlov, S., Wilson, C. P., Mathers, P. H., Lewandoski, M., unpublished manuscript. Rx is essential for lens induction and neural retinal cell fate.

Walther, C., Gruss, P., 1991. Pax-6, a murine paired box gene, is expressed in the developing CNS. Development. 113, 1435-49.

Wanaka, A., Milbrandt, J., Johnson, E. M., Jr., 1991. Expression of FGF receptor gene in rat development. Development. 111, 455-68. 
Wawersik, S., Purcell, P., Rauchman, M., Dudley, A. T., Robertson, E. J., Maas, R., 1999. BMP7 acts in murine lens placode development. Dev Biol. 207, 176-88.

Westenskow, P., Piccolo, S., Fuhrmann, S., 2009. Beta-catenin controls differentiation of the retinal pigment epithelium in the mouse optic cup by regulating Mitf and Otx2 expression. Development. 136, 2505-10.

Winkler, S., Loosli, F., Henrich, T., Wakamatsu, Y., Wittbrodt, J., 2000. The conditional medaka mutation eyeless uncouples patterning and morphogenesis of the eye. Development. 127, 1911-9.

Yang, X. J., 2004. Roles of cell-extrinsic growth factors in vertebrate eye pattern formation and retinogenesis. Semin Cell Dev Biol. 15, 91-103.

Yayon, A., Klagsbrun, M., Esko, J. D., Leder, P., Ornitz, D. M., 1991. Cell surface, heparin-like molecules are required for binding of basic fibroblast growth factor to its high affinity receptor. Cell. 64, 841-8.

Yayon, A., Zimmer, Y., Shen, G. H., Avivi, A., Yarden, Y., Givol, D., 1992. A confined variable region confers ligand specificity on fibroblast growth factor receptors: implications for the origin of the immunoglobulin fold. EMBO J. 11, 1885-90.

Yu, J., Vodyanik, M. A., Smuga-Otto, K., Antosiewicz-Bourget, J., Frane, J. L., Tian, S., Nie, J., Jonsdottir, G. A., Ruotti, V., Stewart, R., Slukvin, II, Thomson, J. A., 2007. Induced pluripotent stem cell lines derived from human somatic cells. Science. 318, 1917-20.

Zaghloul, N. A., Moody, S. A., 2007. Alterations of rx1 and pax6 expression levels at neural plate stages differentially affect the production of retinal cell types and maintenance of retinal stem cell qualities. Dev Biol. 306, 222-40.

Zamora, B. G., Mathers, P. H., submitted manuscript. Rx is required for specification of the neural retina, independent of optic cup morphogenesis and lens induction.

Zhang, L., El-Hodiri, H. M., Ma, H. F., Zhang, X., Servetnick, M., Wensel, T. G., Jamrich, M., 2003. Targeted expression of the dominant-negative FGFR4a in the eye using Xrx1A regulatory sequences interferes with normal retinal development. Development. 130, 4177-86.

Zhang, L., Mathers, P. H., Jamrich, M., 2000. Function of Rx, but not Pax6, is essential for the formation of retinal progenitor cells in mice. Genesis. 28, 135-42.

Zhang, X. M., Yang, X. J., 2001. Temporal and spatial effects of Sonic hedgehog signaling in chick eye morphogenesis. Dev Biol. 233, 271-90.

Zhao, S., Hung, F. C., Colvin, J. S., White, A., Dai, W., Lovicu, F. J., Ornitz, D. M., Overbeek, P. A., 2001. Patterning the optic neuroepithelium by FGF signaling and Ras activation. Development. 128, 5051-60.

Zhao, S., Overbeek, P. A., 1999. Tyrosinase-related protein 2 promoter targets transgene expression to ocular and neural crest-derived tissues. Dev Biol. 216, 154-63.

Zhao, S., Rizzolo, L. J., Barnstable, C. J., 1997. Differentiation and transdifferentiation of the retinal pigment epithelium. Int Rev Cytol. 171, 225-66.

Zhao, S., Thornquist, S. C., Barnstable, C. J., 1995. In vitro transdifferentiation of embryonic rat retinal pigment epithelium to neural retina. Brain Res. 677, 300-10.

Zhu, C. C., Dyer, M. A., Uchikawa, M., Kondoh, H., Lagutin, O. V., Oliver, G., 2002. Six3-mediated auto repression and eye development requires its interaction with members of the Grouchorelated family of co-repressors. Development. 129, 2835-49.

Zuber, M. E., Gestri, G., Viczian, A. S., Barsacchi, G., Harris, W. A., 2003. Specification of the vertebrate eye by a network of eye field transcription factors. Development. 130, 5155-67.

Zuber, M. E., Perron, M., Philpott, A., Bang, A., Harris, W. A., 1999. Giant eyes in Xenopus laevis by overexpression of XOptx2. Cell. 98, 341-52. 